\title{
Review \\ Breath Analysis: A Promising Tool for Disease Diagnosis-The Role of Sensors
}

\author{
Maria Kaloumenou ${ }^{1}$, Evangelos Skotadis ${ }^{1, *(D)}$, Nefeli Lagopati ${ }^{2}$, Efstathios Efstathopoulos ${ }^{2}$ D \\ and Dimitris Tsoukalas ${ }^{1}$ (D)
}

1 Department of Applied Physics, National Technical University of Athens, 15780 Athens, Greece; mariakaloumenou1@gmail.com (M.K.); dtsouk@central.ntua.gr (D.T.)

2 Medical School, National and Kapodistrian University of Athens, 75, Mikras Asias Str., Goudi, 11527 Athens, Greece; nlagopati@med.uoa.gr (N.L.); stathise@med.uoa.gr (E.E.)

* Correspondence: evskotad@central.ntua.gr

check for updates

Citation: Kaloumenou, M.; Skotadis, E.; Lagopati, N.; Efstathopoulos, E.; Tsoukalas, D. Breath Analysis: A

Promising Tool for Disease

Diagnosis-The Role of Sensors.

Sensors 2022, 22, 1238. https://

doi.org/10.3390/s22031238

Academic Editor: James Covington

Received: 31 December 2021

Accepted: 1 February 2022

Published: 6 February 2022

Publisher's Note: MDPI stays neutral with regard to jurisdictional claims in published maps and institutional affiliations.

Copyright: (c) 2022 by the authors. Licensee MDPI, Basel, Switzerland. This article is an open access article distributed under the terms and conditions of the Creative Commons Attribution (CC BY) license (https:/ / creativecommons.org/licenses/by/ $4.0 /)$.

\begin{abstract}
Early-stage disease diagnosis is of particular importance for effective patient identification as well as their treatment. Lack of patient compliance for the existing diagnostic methods, however, limits prompt diagnosis, rendering the development of non-invasive diagnostic tools mandatory. One of the most promising non-invasive diagnostic methods that has also attracted great research interest during the last years is breath analysis; the method detects gas-analytes such as exhaled volatile organic compounds (VOCs) and inorganic gases that are considered to be important biomarkers for various disease-types. The diagnostic ability of gas-pattern detection using analytical techniques and especially sensors has been widely discussed in the literature; however, the incorporation of novel nanomaterials in sensor-development has also proved to enhance sensor performance, for both selective and cross-reactive applications. The aim of the first part of this review is to provide an up-to-date overview of the main categories of sensors studied for disease diagnosis applications via the detection of exhaled gas-analytes and to highlight the role of nanomaterials. The second and most novel part of this review concentrates on the remarkable applicability of breath analysis in differential diagnosis, phenotyping, and the staging of several disease-types, which are currently amongst the most pressing challenges in the field.
\end{abstract}

Keywords: breath analysis; volatile organic compounds; sensors; nanomaterials; differential diagnosis

\section{Introduction}

Disease diagnosis is conventionally conducted using expensive, time-consuming, invasive techniques, applied by appropriately trained health care professionals. For instance, gastroscopy, laryngoscopy, and coronary angiography are used for gastric cancer (GCa), lung cancer (LC), and myocardial infraction diagnosis, respectively [1]. Other commonly used methods, such as computed tomography [2] or mammography, used for breast cancer (BC) [3], may also be harmful due to radiation exposure. As a result, patient compliance and utilization of such diagnostic methods are remarkably reduced for a significant part of the population. However, disease and especially cancer early-stage diagnosis via effective high-risk population screening, renders treatment easier [4]. For this reason, ameliorated diagnostic methods are imperative.

Metabolomics, one of the '-omics' disciplines that have progressively become a promising diagnostic tool in medical research, offer a comprehensive analysis of the metabolites contained in biological samples by the combination of analytical techniques with bioinformatics [5]. On the other hand, the term volatolomics is referred to the chemical processes that correlate with volatile organic compounds (VOCs) emitted by body fluids [6], such as peripheral blood, urine, and sweat as well as feces, nasal mucous, gaseous skin excretions, and exhaled breath [6-8]. Apart from VOCs (e.g., acetone, isoprene, ethane, pentane), inorganic gases (e.g., $\mathrm{CO}_{2}, \mathrm{CO}$ and $\mathrm{NO}$ ) and non-volatile compounds/exhaled 
breath condensates (e.g., peroxynitrite, cytokines, and isoprostanes) constitute the human breath $[1,9,10]$. Decreased sample complexity, the highly developed appropriate analytical techniques, and the ability of direct or continuous breath analysis using gas sensors render exhaled breath as an exceptional source of gas-biomarkers (VOCs predominantly but also inorganic gases) [8,11]. More than 2000 VOCs have been detected in the exhaled breath [12] and appertain to hydrocarbons, alcohols, aldehydes, ketones, esters [12,13], ethers, carboxylic acids, heterocyclic hydrocarbons [12], aromatic compounds, nitriles [12,13], sulfides, and terpenoids [12] and may be endogenous or exogenous.

Exogenously originated VOCs are correlated with the environment and the habits of the person [14]. VOCs related with cleaning fluids, personal care products, plastic-related VOCs [14], blazes, or air pollution due to industrial/transport gas emissions [12] enter human organism through extended inhalation and are excreted via exhaled breath. Smoking [15], food habits and food supplements, drinks, or medication also consist important sources of VOCs [14,15]. Other important confounding factors affecting the profile of exhaled VOCs are age, gender, ethnicity, living place, and lifestyle $[15,16]$. Consequently, immediate and recent environmental exposure should be taken into consideration during breath analysis [14].

Endogenously created VOCs comprise high vapor pressure (body and room temperature (RT)) (fragments of [14]) byproducts of normal or pathophysiological metabolic pathways $[7,14,17]$, as well as of microbiome metabolism [14]. They are produced either in the airway region or in other parts of human body, representing the metabolism of the whole organism. In the first case, VOCs are released in the exhaled breath in a direct way [17]. In the second case, produced VOCs enter and circulate in the bloodstream, and, during gas exchange in the alveoli or the airways, excretion to the exhaled breath occurs $[15,17]$ via the alveolar pulmonary membrane $[9,10]$. Depending on blood solubility, VOCs are exchanged in different sites of the respiratory tract. Nonpolar VOCs with poor blood solubility (blood-air partition coefficient $\left(\lambda_{b: a}\right)<10$ ) are exchanged in the alveoli, in contrast to blood soluble VOCs $\left(\lambda_{b: a}>100\right)$ that are exchanged in lung airways. VOCs of intermediate solubility $\left(10<\lambda_{b: a}<100\right)$ undergo pulmonary gas exchange in both sites [12,15]. Oxidative stress, lipid peroxidation, and reactions catalyzed by cytochrome p450 (CYP450), and liver enzymes are the main biochemical processes correlated with endogenous VOCs $[15,18,19]$. The correlation of oxidative stress and airway inflammation with exhaled VOCs is summarized in Figure 1. Different VOC classes are potentially correlated with different biochemical reactions and exogenous sources.

VOCs of exhaled breath are regarded as normal [12]. However, concentration differences for some exhaled VOCs could potentially be associated with an abnormal condition of the body [12], as the metabolic processes producing the VOCs are altered in a distinctive way by different diseases $[1,10]$. Disease-related concentration alternations conventionally concern a group of VOCs rather than a single compound [17]. Apart from this, the concentration of a single compound may vary due to more than one pathophysiological processes, thus being non-specific [20]. Consequently, diagnosis of complex, heterogeneous diseases is scarcely achieved by the recognition of one characteristic stand-alone VOC $[7,20]$. A mixture of exhaled VOCs, called VOC pattern or 'breathprint', consists the signature of a specific disease, correlating with the underlying pathophysiology; this pattern should therefore be recognized so as to achieve disease diagnosis $[7,17,20]$.

Thus, the analysis of the exhaled breath holds a great promise for non-invasive early disease diagnosis [1,21]. Several diseases are investigated via breath analysis [7] for potential diagnosis, the main types of which are presented on Table 1. Specifically in the case of infections by specific species or strains of bacteria, the combination of bacteria-derived VOCs [22] and VOCs produced by the host due to immune response to bacterial antigens as well as VOCs formed due to the host response to bacterial products/metabolites (and vice versa) is detected. Differentiation of the origin of those VOCs is not clinically important [23]. Recently, research interest focused on the diagnosis of SARS-CoV-2 viral infection via breath analysis with remarkable results [24-26], employing a diagnostic test, i.e., "BreFence Go 
COVID-19 Breath Test System" developed by Breathonix [27] and "TracieX Breathalyser" developed by Silver Factory Technology, which have been already provisionally approved by the HSA [28,29]. Apart from early diagnosis, screening of high-risk populations and assessment of therapy efficiency can be permitted using breath analysis, due to being an inexpensive [6] and rapid method, characterized by increased patient compliance [30].

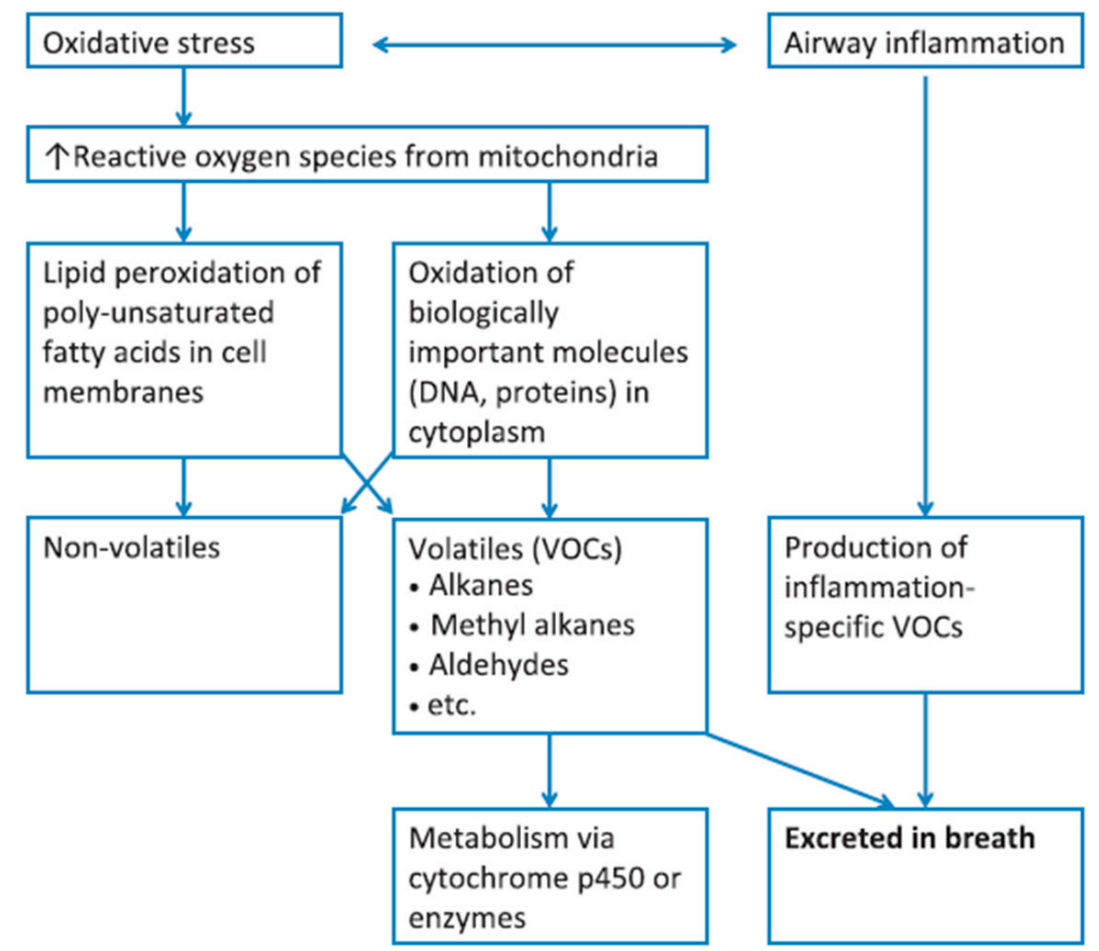

Figure 1. Diagram summarizing the correlation of VOCs present in the exhaled breath, with oxidative stress and inflammatory conditions; metabolic breakdown of larger molecules leads to the formation of exhaled VOCs. Reprinted with permission from ref. [17]. Copyright @ 2012 John Wiley \& Sons Ltd.

Table 1. Diseases investigated for diagnosis using breath analysis.

\begin{tabular}{ccc}
\hline Disease Type & Diseases & Ref. \\
\hline Respiratory & $\begin{array}{c}\text { Asthma, COPD, obstructive sleep apnea syndrome, } \\
\text { pulmonary arterial hypertension, cystic fibrosis }\end{array}$ & {$[19]$} \\
\hline Malignant & Lung, gastric, head and neck, breast, colon, prostate cancer & {$[15]$} \\
\hline Neurodegenerative & Alzheimer's disease, Parkinson's diseases, multiple sclerosis & {$[15]$} \\
\hline Metabolic & Diabetes, hyperglycemia & {$[12,31]$} \\
\hline Bacterial infections & $\begin{array}{c}\text { Upper respiratory tract infection, Mycobacterium tuberculosis, } \\
\text { Pseudomonas, Helicobacter pylori infection }\end{array}$ & {$[22,32]$} \\
\hline Viral infections & SARS-CoV-2 & {$[24-26]$} \\
\hline COPD: Chronic obstructive pulmonary disease.
\end{tabular}

For the analysis of exhaled VOCs and disease diagnosis, two different methods can be used; analytical techniques and sensors. Gas chromatography combined with mass spectrometry (GC-MS) comprises the gold-standard method for the analysis of VOCs patterns in exhaled breath $[1,17,33]$. Both quantitative analysis (characterized by high sensitivity in the ppb to ppt range [1]) and qualitative analysis (providing information concerning the potential metabolic disease pathways [33]) can be achieved [4,17]. For the detection of very low concentrations of VOCs, the pre-concentration of the breath sample is imperative. Pre-concentration techniques commonly combined with GC-MS include thermal desorption [31,33] (using sampling bags/sorbent tubes [33], mainly Tenax tubes [31]), headspace 
solid-phase microextraction (HS-SPME) [10,31] (using silica fibers, coated with polymeric nanofilm, mainly Carboxen (CAR)/Polydimethylsiloxane (PDMS) [31]) and the needle trap device $[31,34]$ (sorbent polymer-CAR, PDMS, and/or divinylbenzene (DVB)—packed in a needle [31]). Apart from GC-MS, selected ion flow tube-mass spectrometry (SIFT-MS), ion mobility spectrometry (IMS), proton transfer reaction-mass spectrometry (PTR-MS) [17,35], proton reaction transfer time-of-flight mass spectrometry (PRT-TOF-MS) [1], GC coupled with ion mobility spectrometry (GC-IMS), and flame ionization detector (GC-FID) [31] are also common techniques for breath analysis. However, spectrometry and spectroscopic methods exhibit important limitations [15], such as the use of bulky and expensive equipment by appropriately trained personnel, while the analysis is also time-consuming $[15,36]$, providing no real-time results [33]. In addition, pre-concentration methods, required before the analysis, could potentially lead to sample loss/contamination $[34,36]$. Thus, despite the advantages of those analytical techniques, their use in clinical practice for point-of-care [37] or screening [33] is limited.

During recent years, however, sensors and e-Noses, have exhibited the prospect of becoming strong diagnostic tools via breath analysis and are rising up to the existing clinical challenges [34,35,37]. Gas sensors comprise inexpensive and simple [12,34,37] easy-to-use devices that are small in size and thus portable [12,34]. In addition, short response time and direct acquisition of results, as well as short sensor recovery time [12], render gas sensors attractive for point-of-care and personalized screening, diagnosis, and disease follow-up [34]. Exhaled gas-target analysis using sensors can be achieved by two different approaches (Figure 2). In the first case, a targeted approach is applied, using a selective mechanism [7,14]. The target is recognized by a selective chemical sensor, designed to measure this single compound in a complex mixture, based on lock-key mechanism [7]. Such selective sensors have been developed for $\mathrm{NO}, \mathrm{NH}_{3}$, acetone, $\mathrm{H}_{2} \mathrm{O}_{2}[6,15], \mathrm{H}_{2} \mathrm{~S}$, and $\mathrm{CH}_{3} \mathrm{SH}$ [15]. On the other hand, the detection of a unique gas-target pattern, rather than a single exhaled compound, is also possible. Semi-selective/ cross-reactive sensors are artificially intelligent nano-arrays [15] mimicking natural sensing systems [38,39] and are also called "electronic noses" (e-Noses), "artificial olfactory systems" (gas analytes), or "electronic tongues" (liquid analytes). The array consists of distinctive sensors that respond to all/large part of the components of a complex mixture [38], at the same time, in a complementary way [39]. Due to their diversity each individual sensor of the array responds differently (yet not chemically selectively) to a given mixture. Statistical pattern-recognition algorithms and classification techniques are used for the establishment of analyte-specific response patterns, combining the responses of the sensor array elements [38]. It is worth noting that even analytical techniques, e.g., GC-MS, are progressively used for the analysis of total patterns of VOCs, instead of targeting a stand-alone biomarker [17].

Multivariate data analysis, a fundamental tool in breath analysis, improves the human perception of experimental data [16]. Response data obtained after sensor array exposure to a complex chemical mixture are processed by multivariate data analysis [40] in order to assess the discriminating ability of the sensor array [16,41], as well as for the elimination of potential confounding variables (i.e., environmental temperature and humidity) [40]. Multivariate data analysis is also useful for breath analysis using analytical techniques, permitting the identification of the most discriminant VOCs between the different groups studied [32]. Numerous multivariate analysis methods are used in e-Nose systems, including canonical discriminate analysis (CDA), partial least squares regression (PLS regression), discriminant function analysis (DFA), and principal component analysis (PCA) (Figure 3) [40]. PCA comprises the most commonly used method in e-Nose systems [40], while DFA is also frequently used. 


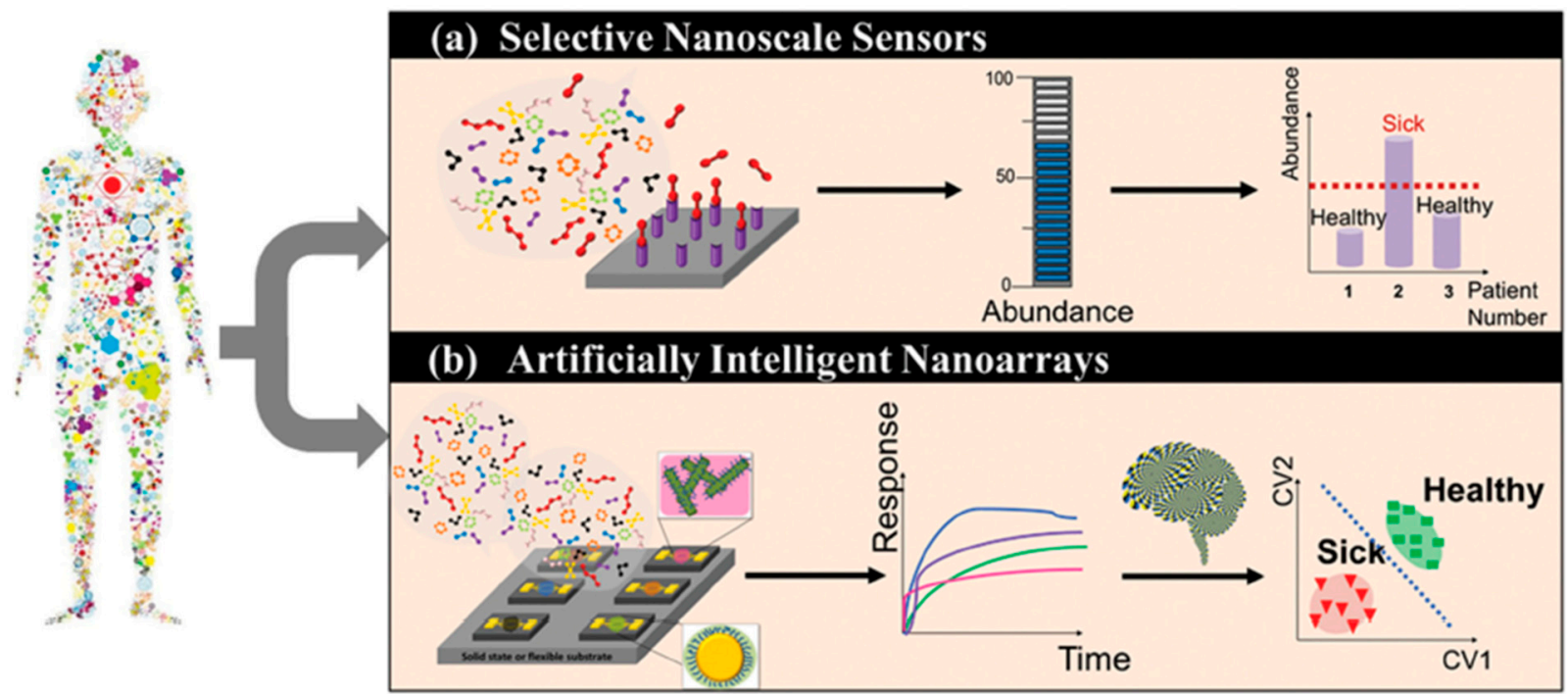

Figure 2. Schematic representation of the working principle of selective sensors and artificially intelligent cross-reactive sensor arrays. Selective sensors contain highly selective elements in order to detect a specific gas-analyte in the presence of a composite gas-mixture. Cross-reactive arrays feature sensors that are sensitive to the majority of the gases present in the gas-mixture. In any case, detecting analyte concentration above a critical value leads to the differentiation between sick and healthy subjects. The response of gas-sensing arrays can be then processed by employing artificial intelligence, machine-learning, and pattern recognition techniques. Reprinted with permission from Ref. [6] Copyright (c) 2015 Wiley-VCH Verlag GmbH \& Co. KGaA, Weinheim.
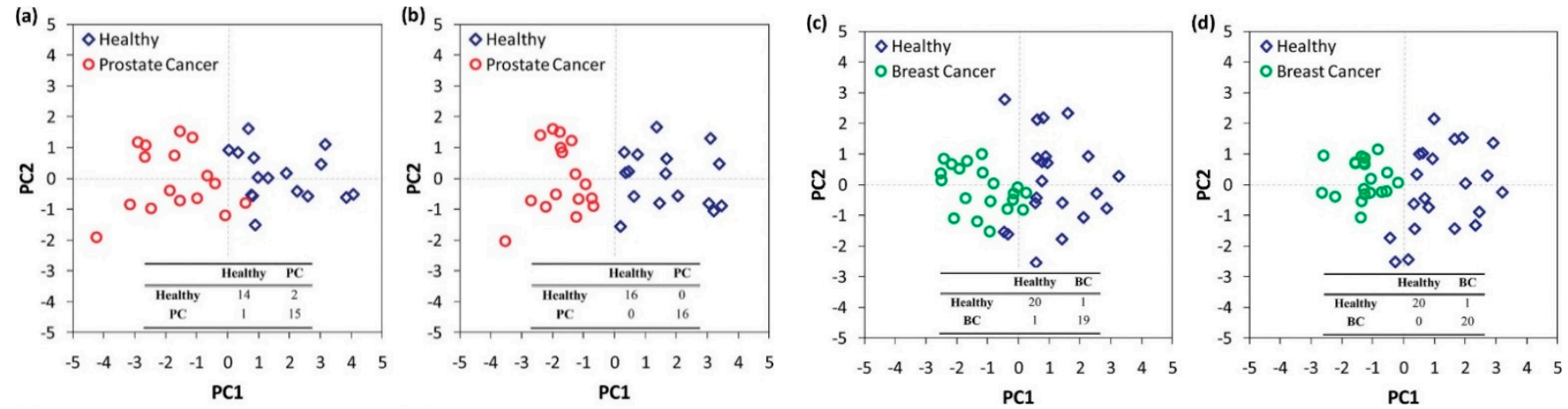

Figure 3. Statistical analysis of the response of a nanomaterial-based, cross-reactive chemiresistor for real-world samples of sick and healthy subjects. The use of PCA permits the differentiation of the groups. Notably, relative humidity compensation reduces the dispersion of different clusters thereby improving the discrimination between healthy and sick subjects. Representative 2D breath-analysis PCA plots for prostate cancer diagnosis: (a) without relative humidity compensation; (b) with relative humidity compensation. PCA plots for breast cancer diagnosis: (c) without relative humidity compensation; (d) with relative humidity compensation. Adapted with permission from Ref. [42] Copyright (C) 2012, American Chemical Society.

PCA is an unsupervised learning technique in which the multidimensional data space is reduced to its main components [16]. Linear combinations of original data (i.e., sensor values) capturing the maximum variance between all data points are acquired [16,42], leading to a reduced set of variables [41], called principal components (PCs). The differentiation of the PCs maintaining most of the original data information from the PCs with the minimum effects, which are excluded, is achieved by employing an appropriate algorithm [41]. PCs define new orthogonal axes for the representation of multidimensional data only in two or three dimensions [16,42]. Thus, a visualized statistical analysis is obtained, 
permitting discrimination of otherwise entangled data [41]. PC1 is characterized by the greatest response variance, while the magnitudes of variance are diminished from PC2 to PC3 and so on [16,42]. DFA is a linear supervised pattern recognition method, also used for multidimensional experimental data reduction [43]. DFA aims to separate known data groups to the best possible extent [44]. The determination of the discrimination classes is conducted prior to the analysis [43]. Input variables are linearly combined to achieve the maximum variance between classes and the minimum variance for each class [43,44]. DFA output is a set of canonical variables (CVs) that fulfill the above two requirements. The first $\mathrm{CV}$ is the most powerful discriminating dimension [43].

In order to evaluate sensor array suitability for disease diagnosis and to assess the ability to correlate VOCs with the appropriate disease, machine learning techniques [43] such as support vector machines (SVM), k-nearest neighbor (k-NN), and artificial neural networks (ANN) [40] are also needed. Machine learning techniques and artificial intelligence in general aim at enabling machines to mimic a specific behavior by designing and developing algorithms based on empirical data that are representative of the relation between the observed attributes [45]. The automated "learning" of complex patterns and intelligent decision-making based on current data comprise one of the principal research fields in machine learning [45] and are especially useful in the development of e-Noses for disease diagnosis. ANN is a commonly used machine learning technique, inspired by the human nervous system. The most discriminant sensing features are firstly determined and comprise the input data of the ANN. The input data are connected with the output (i.e., the classification of samples to specific disease) by the ANN, using a set of appropriate functions. The classification from the available inputs is improved by optimizing specific parameters, such as the number of neurons (calculation centers) that are responsible for the system calculations [43].

A number of requirements have to be fulfilled for the use of a sensor in breath analysis and therefore disease diagnosis. Reproducibility [6], high sensitivity, and good resolution [15], are three fundamental parameters. Low limit of detection (LOD) [13,15] (ppb [38]), wide range of response [38], and increased selectivity are also of great importance, in order to detect the exhaled VOCs in the presence of water vapor found in the humidified clinical samples [15]. The stable baseline in the absence of gas-target biomarkers [6], short response, and recovery times are imperative [13,15], while full recovery of the sensor after analyte removal is also essential. Alternatively, disposable sensors that are simple, cost-effective, and therefore suitable for mass-production can be used [15]. Last but not least, operation at RT is important [38].

In the context of this fast-growing field, novel nanomaterials and sensing devices are constantly being developed. The first part of this review aims to provide an up to date overview of the main classes of sensors investigated for the analysis of exhaled gas-analytes (i.e., VOCs, inorganic gases) for disease diagnosis. After highlighting the importance of nanomaterials, examples of applications of either selective or cross-reactive self-developed and commercial sensing devices are discussed. The second and final part of the paper discusses and highlights the capacity of breath analysis towards differential diagnosis, disease staging and phenotyping. There is currently a strong and urgent need for clinically applicable, portable and non-invasive diagnostic tools that can lead to the distinction of different diseases with similar symptoms; the current review paper evaluates the potential of sensing devices of varying technology, as well as analytical techniques, to serve as reliable breath analysis tools for differential diagnosis while it also discusses advantages and weaknesses that need to be addressed.

\section{The Role of Nanomaterials}

More recently, research interest has notably focused on the development of chemical sensors incorporating nanomaterials [6,46-48]. Despite their inability for qualitative analysis in complex samples and poor quantitative performance as well as humidity sensitivity and relatively short life, nanomaterial-based gas sensors possess major advantages that 
render them exceptionally promising [12]. The small dimensions of nanomaterials (typically 1-100 nm) increase their surface-to-volume ratio and the interaction sites [6,15]; those novel interfaces $[38,49]$ lead to high sensor sensitivity as well as small response/recovery times $[6,15]$. Higher specificity for a desired analyte is also achieved, by sensibly selecting the physical/chemical properties of the nanomaterials $[6,15]$. Those properties are attributed to the size, shape, and composition of nanomaterials [38] and can therefore be easily modified [6,15]. On the other hand, similar dimensions between nanomaterials and biomolecules render the former attractive for application in medical diagnostic devices [38]. Last but not least, the combination of nanomaterials with different properties (easily accomplished by large-scale manufacturing methods) permits a synergistic sensing ability for the device [6,15], along with simple, portable and energy-efficient operation [6]. As it will be discussed below, different nanomaterials have been used in several gas sensors for gas-analyte detection, as transduction elements [6].

\subsection{Metallic Nanoparticles (MNPs)}

Noble metals ( $\mathrm{Au}, \mathrm{Ag}, \mathrm{Pt}, \mathrm{Pd})$ possess exceptional chemical, physical and electronic properties [50]. They exhibit increased conductivity [13], mechanical robustness [50], oxidation resistance and, thus, chemical stability $[13,50]$. In the form of nanoparticles (NPs), additional novel properties-attributed to the increased surface area and the domination of quantum-mechanical properties [51] —are exhibited; optical (localized surface plasmon resonance (LSPR) phenomenon) [36] and electrical [51] are amongst the more interesting. The mechanical, optical, and electrical properties of MNPs are composition, size, periodicity and inter-particle distance dependent [51]. Notably, chemical environment-dependence of those properties renders MNPs promising for gas-sensing [36,50].

MNPs are extensively investigated in gas sensors for breath analysis applications and are usually combined with other nanomaterials (e.g., carbon nanotubes (CNTs), graphene, metal oxide semiconductors (MOS)), to form more effective sensing materials, with increased sensitivity and selectivity $[13,36]$. In addition, gas-sensor sensitivity is overall enhanced by the presence of NPs on the surface of other sensing nanomaterials, due to defect formation on which gases can preferably adsorb. Smaller NPs lead to greater surface area, increasing the number of defects and ultimately sensor sensitivity [52]. MNPs can be also functionalized with a variety of organic ligands hence forming thin films with tunable chemical selectivity (molecularly-capped NPs, MCNPs), characterized by controllable inter-particle distance and reproducible production [38].

\subsection{Metal Oxide Semiconductors (MOS)}

MOS are commonly used in different sensor types as sensing materials, for oxidizing or reducing gas detection. Transition MOS (e.g., $\mathrm{NiO}, \mathrm{Cr}_{2} \mathrm{O}_{3}$ ) are more efficient for gas sensing applications than the non-transition $\mathrm{MOS}\left(\mathrm{ZnO}, \mathrm{SnO}_{2}\right)$, due to more than one preferred oxidation states [7]. Increased affinity of MOS for negatively charged oxygen species (e.g., $\mathrm{O}_{2}{ }^{-}, \mathrm{O}^{-}$), in contrast to compound semiconductors (e.g., GaAs) [53], leads to the creation of surface-trapped charge density; in this way electron depletion layers are formed in n-type semiconductors while hole accumulation layers are formed in p-type semiconductors $[7,53]$. Gas interaction with the oxygen species alternates the surface charge density, resulting in a resistance change [7]. Specifically, oxidation of reducing gases by the adsorbed oxygen species leads to electron transfer towards the semiconductor surface. The adsorption of oxidizing gases, on the other hand, leads to the removal of electrons from the semiconductor surface [53].

N-type MOS, e.g., $\mathrm{SnO}_{2}, \mathrm{ZnO}, \mathrm{WO}_{3}, \mathrm{TiO}_{2}, \mathrm{MoO}_{3}, \mathrm{In}_{2} \mathrm{O}_{3}$, and $\mathrm{Fe}_{2} \mathrm{O}_{3}$, as well as p-type MOS, such as $\mathrm{Co}_{3} \mathrm{O}_{4}, \mathrm{CuO}, \mathrm{NiO}, \mathrm{Cr}_{2} \mathrm{O}_{3}$, and $\mathrm{Mn}_{3} \mathrm{O}_{4}$, have been used in gas sensors for disease diagnosis [53]. MOS sensitivity is affected by numerous parameters, such as morphology, porosity, particle size, film thickness and doping of MOS, decoration with noble metals, as well as operation temperature [54]. Consequently, various MOS based gas-sensors of different structures (villi-like [55], nanotubes (NTs) [56], Hemi tubes [57], hierarchical 
fibers [58], nano-sheets [59], or NWs [60]), usually combined with noble MNPs [56-59], 2D-materials [57], or MOS nanoparticles [59,60] have been investigated as diagnostic tools for a variety of diseases. Notably, in the case of semiconducting nanomaterials, CNTs, graphene-based nanomaterials, and MOS have exhibited the highest response towards acetone, thus holding great promise for diabetes diagnosis [53]. However, despite their enticing applications MOS possess important limitations including high temperature operation [13] (150-500 $\left.{ }^{\circ} \mathrm{C}\right)$ and confinement to single gas detection, due to lack of selectivity towards polar-nonpolar compounds [7].

\subsection{Carbon Nanotubes (CNTs)}

CNTs are investigated as gas sensing materials for their interesting electrical, mechanical, optical and thermal properties [13,61], as well as for their compatibility with other nanomaterials for enhanced performance [7]. CNTs are divided into single wall CNTs (SWCNTs) with a diameter in the range of 1-5 nm [50] and multi-wall CNTs (MWCNTs) with a diameter in the range of 5-100 nm [7] and an interlayer spacing of $3.4 \AA$ [50]. Concerning their electrical properties, which are most commonly exploited, SWCNTs act as metallic conductors or semiconductors (depending on the chiral angle between hexagons and tube axis), while MWCNTs behave as metallic conductors, with current density up to $10^{9} \mathrm{~A} / \mathrm{cm}^{2}$ [7].

CNTs-analyte interaction may include Van der Waals or donor-acceptor interactions. However, gas adsorption-provoked charge-transfer is the main sensing mechanism since SWCNTs specifically can function as p-type semiconductors $[7,13,50]$. Oxidizing gasadsorption, such as $\mathrm{NO}_{2}$, decreases sensing-layer resistance, due to electron withdrawing by the gas. In contrast, reducing analyte-adsorption, such as $\mathrm{NH}_{3}$, increases resistance [13] Remarkably, in those cases SWCNTs act as both the sensing element and the transducer [50].

Both categories of CNTs, however, are characterized by lack of chemical selectivity, high $\mathrm{H}_{2} \mathrm{O}$ affinity, low sensitivity for nonpolar compounds [38], and slow recovery [7]. For this reason, functionalization is imperative. Decoration with MNPs for enhanced selectivity is discussed in the literature [38]. More importantly, CNTs are commonly functionalized with analyte specific entities, covalently (esterification, amidation of carboxyl groups added to CNTs) or non-covalently (supramolecularly, via Van der Waals, and $\pi-\pi$ interactions) [50]. Modification of CNTs with non-polymeric organic layers [38] (e.g., polycyclic aromatic hydrocarbons [62]) or polymers [50] have been used for the development of effective cross-reactive gas sensors as diagnostic tools via breath analysis.

\subsection{Nanowires}

NWs have also been investigated for gas sensing systems with SiNWs being the most common while MOS-NWs are also used. SiNWs possess interesting optical and electrical properties and compatibility with the technologies currently used in microelectronics [38], acting as n-type or p-type semiconductors and with a maximum operation temperature of $150{ }^{\circ} \mathrm{C}$. Conductivity is modified depending on the nature of the gaseous analyte (oxidizing/reducing) and the type of SiNWs (n-/p-type), while the adsorption of charged oxygen species $\left(\mathrm{O}_{2}{ }^{-}\right.$at $\left.150^{\circ} \mathrm{C}\right)$ determines the NWs conductance properties [63]. VOC polarity is of great importance while the physical adsorption of polar molecules via Van der Waals/electrostatic interactions affects the surface potential [7,63].

Modification of SiNWs properties is feasible [7]; SiNWs doping determines sensor sensitivity by affecting the number of charge carriers of the NWs, similarly to MOS [63]. Chemical functionalization with appropriate molecular ligands $[43,64]$ on the other hand enhances sensor selectivity since non-polar vapor detection by pristine SiNWs is inefficient in contrast to polar VOCs [7]. Molecular modification also serves in the fabrication of cross-reactive arrays for the detection of potential VOC biomarkers $[38,43,65,66]$. 


\subsection{D-Materials}

2D-materials (e.g., graphene, $\mathrm{MoS}_{2}, \mathrm{MoSe}_{2}, \mathrm{WSe}_{2}$, and $\mathrm{NbSe}_{2}$ ) have been investigated for gas sensor development. Their main advantage is low-power sensor operation at RT, while they are characterized by unique electrical properties [13] and large surface-tovolume ratio $[13,67]$. The latter renders 2D materials excellent candidates for gas sensing applications [67]. Gas analyte adsorption at multiple active sites, on the edge and surface defects of the material, changes its electronic properties. Similarly to CNTs charge-transfer interactions comprise the basis of the gas sensing mechanism [13]. Gas adsorption changes the resistivity of the sensor, depending on the type of gas (reducing/oxidizing) and semiconducting 2D-material (p-/n-type) [67].

Transition metal dichalcogenides and graphene in particular are extensively used in gas sensing systems, facilitating disease diagnosis via breath analysis [7,53,67]. Transition metal dichalcogenides (TMDs) ( $\mathrm{MX}_{2}, \mathrm{M}$ : transition metal, $\mathrm{X}: \mathrm{S}, \mathrm{Se}$, or Te) such as $\mathrm{MoS}_{2}$, $\mathrm{MoSe}_{2}, \mathrm{Wse}_{2}, \mathrm{NbSe}_{2},[13] \mathrm{WS}_{2}, \mathrm{SnS}_{2}$, and $\mathrm{SnSe}_{2}$ [68], possess great structural and optical features [69] and tunable semiconducting properties [68] that render them promising materials for e-Noses. TMDs are able to create flexible structures and to operate at low temperatures; however, the increased response/recovery times and the inability to fully recover after analyte exposure are important limitations [68]. To cope with these issues, catalytic MNPs which also improve selectivity can be employed [68,69].

Graphene is characterized by exceptional electrical, thermal and mechanical properties, with low resistivity of $10^{-6} \Omega \cdot \mathrm{cm}$ at RT and large surface-area of $2630 \mathrm{~m}^{2} / \mathrm{g}$ (higher than CNTs, $1000 \mathrm{~m}^{2} / \mathrm{g}$ [53]) being the most promising 2D material for gas sensing. Graphene can also behave as a p-type semiconductor while chemical functionalization can be easily achieved $[7,68]$. Remarkably, graphene has been used for selective rather than cross-reactive applications. Graphene derivatives have also been used for gas sensing applications. Graphene oxide (GO) is characterized by a lower cost of production, however its application in electronic devices is hindered by reduced conductivity. Reduced graphene oxide (RGO) on the other hand exhibits tunable conductivity along with greater gas responses than graphene since the presence of oxygen functional groups enhances gas adsorption $[67,68]$. It is noteworthy that the use of RGO-based gas sensors needs further investigation as the underlying gas-oxygen groups interactions are yet to be explained [53].

2D materials are usually combined with various nanomaterials, for enhanced sensing [7]. The poor sensing performance of pristine 2D materials is primarily attributed to the weak interactions between the adsorbed molecules and the sensing layer [13]. Graphene and RGO combined with MOS [57,70] and MNPs [59] may serve in the development of gas sensing diagnostic tools.

\subsection{Hybrid Materials}

The combination of distinctive nanomaterials so as to form hybrid materials is extensively investigated for disease diagnosis via gas-sensing, as previously discussed. The combination of different nanomaterials improves sensing selectivity and sensitivity $[7,13,38]$ due to their synergic action, rendering hybrid materials particularly promising [49]. Combinations of CNTs with MNPs, 2D materials with MOS or MNPs as well as MNPs [41,71], MOS NPs, or CNTs with polymers are only some of the reported combinations [7]. Particularly polymers are commonly used for achieving chemical selectivity. Concerning (semi-)conducting polymers (e.g., polyaniline, polypyrrole, polythiophene), conductivity changes can be attributed to electrically-active analyte adsorption, due to redox interactions with either the backbone or the incorporated particles in the case of composites [50]. Nonconducting polymers are also used as sensing films, incorporating conductive NPs such as CNTs [50] or carbon black particles [72] for semi-selective analyte absorption; this leads to mass/conductivity changes of the polymeric nano-composites. Finally, molecularly imprinted polymers (MIPs) are a new class of sensing films in which artificial analyte-specific cavities have been created, for specific molecular recognition $[73,74]$. 


\section{Types of Nanomaterial-Based Sensors in Breath Analysis}

Different sensor types have exhibited promising diagnostic ability via breath analysis [7]. In the following part nanomaterial-based gas sensors are categorized depending on the transduction method. Examples of both selective and cross-reactive applications are presented, in an attempt to provide an up-to-date overview.

\subsection{Chemiresistors}

Chemiresistors are one of the most promising types of gas-sensors. They are characterized by simple configuration and working principle [13], increased reliability, decreased size and weight, while they are suitable for automatic packaging in wafer level thus permitting the mass-production of portable, on-chip sensor arrays [51]. Two pairs of electrodes are connected with an overlying sensing layer that is either semiconducting or metallic. Interdigitated electrodes (IDEs) are commonly used for enhanced sensing response [13]. Sensor-resistance changes upon gas exposure, while constant current/potential is applied between the two electrodes [13,51]. Quantitative analysis of the analytes is possible by measuring the change of resistance/current [13]. A schematic representation of typical chemiresistors is presented in Figure 4.

(A)

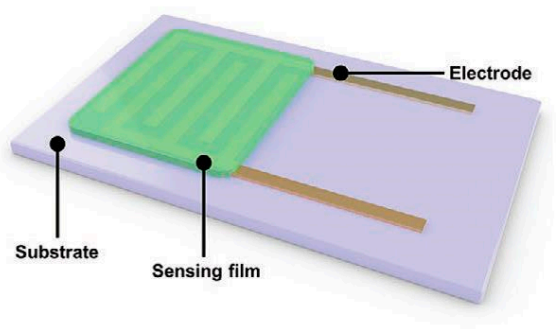

(B)

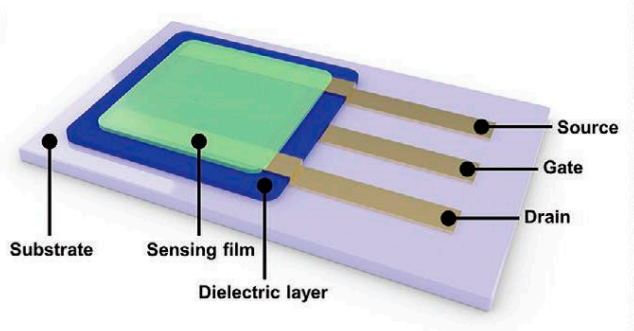

(C)

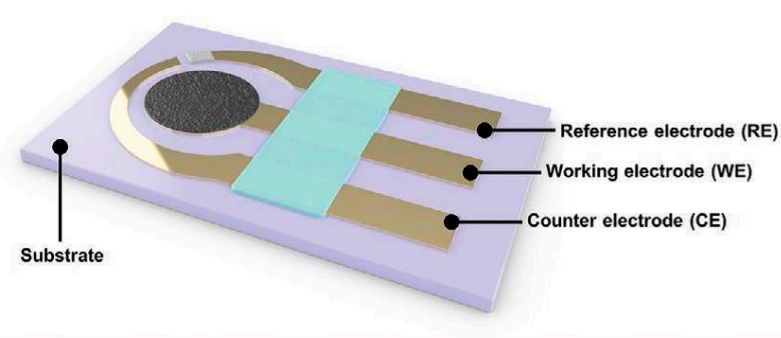

Figure 4. Schematic representation of (A) a chemiresistor under a constant bias and with an overlying metallic or semiconducting sensing layer which acts as the sensitive gas-sensing layer; (B) a fieldeffect transistor where the conductivity of the channel is sensitive to gas-analytes exposure; (C) an electrochemical sensor (potentiometric, amperometric, or conductometric) composed of a working (sensing) electrode on which the analyte reacts (redox reaction), a counter electrode (with respect to which electrical signal is measured) and a reference electrode of "reference" potential. Reprinted with permission from Ref. [13] Copyright @ 2021 Wiley-VCH GmbH.

\subsubsection{Selective Applications}

Concerning single analyte detection, nanomaterial-based sensing films such as MOS, CNTs and hybrid materials are commonly used. MOS-based chemiresistors, in particular, (e.g., $\mathrm{WO}_{3}$ [55], $\mathrm{SnO}_{2}$, or $\mathrm{Cu}_{2} \mathrm{O}$ [36]) are common in the detection of oxidizing/reducing gases [75]. Moon et al. [55] used a chemiresistive porous thin film composed of villi-like $\mathrm{WO}_{3}$ structures and achieved selective and sensitive detection of the asthma biomarker $\mathrm{NO}$ in presence of ethanol, acetone, $\mathrm{NH}_{3}$ and $\mathrm{CO}(80 \%$ relative humidity $(\mathrm{RH}))$ at $150-250{ }^{\circ} \mathrm{C}$, with a LOD of $88 \mathrm{ppt}$ which is far lower than exhaled NO. Selective arrays of MOS sensors have been also used; highly porous $\mathrm{Pt}, \mathrm{Si}, \mathrm{Pd}$, or Ti doped $\mathrm{SnO}_{2} \mathrm{NPs}$ have selectively detected low levels of formaldehyde ( $3 \mathrm{ppb}$ ) in $90 \% \mathrm{RH}$, as well as in synthetic breath 
samples containing acetone, ethanol and $\mathrm{NH}_{3}$, aiming at LC diagnosis [76]. Concerning CNT-based chemiresistors, $\mathrm{NO}_{2}$ selective detection has been reported, using a random or an aligned network of SWCNT, with the former achieving a lower LOD in the range of ppt [77]. Ethanol detection by vertically-aligned CNTs has also been achieved [78].

Among hybrid materials, MNPs-functionalized nanomaterials are commonly investigated. Highly porous thin-wall $\mathrm{SnO}_{2}$ fibers of hierarchical structure composed of $\mathrm{Pt}$ NPs-decorated $\mathrm{SnO}_{2} \mathrm{NTs}$, have been investigated for acetone and toluene detection as diabetes and LC biomarkers respectively; increased sensor responses for both analytes were permitted, due to MNPs-functionalization [58]. More recently, Pt-decorated $\mathrm{Zn}_{2} \mathrm{SnO}_{4}$ hollow octahedra were used for enhanced selective detection of acetone, in ppb levels, adequate for breath analysis applications and diabetes diagnosis [79]. In another application, $\mathrm{Au}$ NPs-decorated SWCNTs were used for selective detection of $\mathrm{H}_{2} \mathrm{~S}$ (halitosis biomarker) in the presence of $\mathrm{NH}_{3}$ and $\mathrm{NO}$, with a LOD of 3 ppb [80]. Au NPs functionalization permitted increased sensitivity for the desired analyte [80].

Other types of combinations of nanomaterials, including MOS, CNTs, or 2D materials, have been also studied. The respective halitosis and diabetes biomarkers $\mathrm{H}_{2} \mathrm{~S}$ and acetone for example, have been detected using $\mathrm{rGO}$ nanosheets-functionalized $\mathrm{SnO}_{2} \mathrm{NFs}$ with increased selectivity and sensitivity [81] as well as using $\mathrm{GO}-\mathrm{WO}_{3}$ and thin-layer graphite- $\mathrm{WO}_{3}$ Hemi tubes in the presence of $\mathrm{CO}, \mathrm{NH}_{3}, \mathrm{NO}$, ethanol, and pentane; high responsiveness and sensitivity and a lower LOD for $\mathrm{H}_{2} \mathrm{~S}$ were achieved in the latter study [57]. The combination of graphene derivatives with conducting polymers was also reported; porous and mechanically improved films composed of GO and polypyrene for instance, have been used for rapid toluene detection [82]. In the field of CNTs-based hybrid materials, SWCNTs were recently combined with porphyrin-based NFs to form a nano-composite of $1 \mathrm{D}$ nano-architecture that permitted the selective detection of $\mathrm{H}_{2} \mathrm{O}_{2}$, in the simultaneous presence of various gases and VOCs. The enhanced sensitivity, reproducibility, and responsiveness, rendered the sensing film promising for cancer, traumatic brain injury, chronic obstructive pulmonary disease (COPD), and asthma diagnosis [83]. The combination of CNTs with conducting polymers has been reported as well, especially for selective detection of ammonia. Such sensors employ carboxylated-MWCNTs-polypyrrole [84] or polyaniline-MWCNTs nano-composites featuring increased sensitivity in the presence of $\mathrm{H}_{2} \mathrm{~S}, \mathrm{NO}_{2}$ acetone, ethanol, and isoprene [85].

\subsubsection{Cross-Reactive Applications}

Apart from selective applications, chemiresistors have also been extensively investigated for the development of sensor arrays and cross-reactive devices. MOS have been used as components of such sensing systems. Arrays of commercial MOS sensors (TGS), have been used by Binson et al. [86,87] and Marzorati et al. [88] for the differentiation of LC [86-88] and COPD patients from HC, with high accuracies (Table 2). Interestingly, LC diagnosis using an array of TGS sensors has been reported in the literature; notably, 85 realworld breath samples of LC patients have been used, aiming not only at the discrimination between LC and HC but also at before- and after-surgery patients [89]. The aforementioned system could potentially comprise a promising diagnostic and prognostic tool after LC resection surgery, since it was shown that HC and operated patients' breath-prints converged over time Table 2 [89]. It is noteworthy that commercial MOS-based chemiresistive e-Noses have been developed and studied for breath analysis and diagnosis. "Common Invent e-Nose", for instance, has been used for asthma phenotyping and exacerbation prediction [90], while "SpiroNose" [91] and "AeoNose ${ }^{\circledR}$ " [92] have been used for asthma, COPD and LC patients and COPD, LC patients and HC differentiation, respectively. "DiagNose", composed of 12 different doped MOS-sensors, comprises another promising example since it managed to differentiate 10 individuals through their breath samples [93]. Self-developed MOS-chemiresistors, in the form of pellets, based on a mixture of $\mathrm{SnO}_{2}$ and $\mathrm{ZnO}$ nano-powders (9:1) have been effectively used for asthma diagnosis; however, a small number of subjects was included [94]. The combination of various materials for 
sensor-array development was also reported; an on-chip array of parallel $\mathrm{ZnO}, \mathrm{Pd}$, and polypyrrole single NWs for instance has been used for BC biomarkers, permitting increased responsiveness and sensitivity as well as total discrimination of the volatile biomarkers (Table 2) [95].

However, hybrid materials comprise the most extensively used materials for chemiresistive sensor arrays. Noble metal-functionalized MOS have been broadly studied. Halitosis diagnosis using an array of pristine and Pt NPs-decorated $\mathrm{WO}_{3}$ macroporous NFs comprises a representative example (Table 2); increased responses towards $\mathrm{H}_{2} \mathrm{~S}$ were achieved by the $0.042 \mathrm{wt} \% \mathrm{Pt} \mathrm{NPs}_{\mathrm{W}} \mathrm{WO}_{3} \mathrm{NFs}$ [96]. PCA successfully discriminated $\mathrm{H}_{2} \mathrm{~S}$, acetone, toluene, and methyl mercaptan vapors, while real-breath samples of healthy controls (HC) and simulated halitosis breath were effectively classified [96]. Similarly, an array of pristine $\mathrm{Au}$ NPs $(1 / 3 \mathrm{~nm})$ decorated with vertically ordered hematite NTs, has been studied for acetone detection; the desired analyte led to far greater responses, while the studied vapors (acetone, ethanol, $\mathrm{CO}, \mathrm{H}_{2}, \mathrm{NH}_{3}$, toluene, and benzene) were differentiated using PCA [97]. The theoretical acetone LOD of 304 ppt renders Au NPs- $\mathrm{Fe}_{2} \mathrm{O}_{3} \mathrm{NTs}$-based sensor particularly promising for diabetes diagnosis. Cross-sensitivity towards NO and toluene using pristine $\mathrm{WO}_{3}$ NTs (with increased selectivity for NO) and catalyst (Pt and Pd) decorated $\mathrm{WO}_{3} \mathrm{NTs}$ (more selective for toluene) has been also achieved, holding great promise for asthma and LC diagnosis via breath analysis [56]. The development of halitosis and diabetes diagnostic sensors has been attempted by the same group using cross-sensitive, pristine, thin-walled, and Pt NPs-decorated $\mathrm{WO}_{3}$ Hemi tubes, with increased sensitivity towards $\mathrm{H}_{2} \mathrm{~S}$ and acetone respectively [98]. More recently, a $2 \times 4$ array of sensors based on pristine $\mathrm{Pt}, \mathrm{Pd}$, or $\mathrm{Au} \mathrm{NPs}$ decorated $\mathrm{In}_{2} \mathrm{O}_{3}$ and $\mathrm{WO}_{3}$ nano-rods (NRs), was investigated for the detection of acetone, $\mathrm{NO}$, and $\mathrm{H}_{2} \mathrm{~S}$ as diabetes, asthma, and halitosis biomarkers. This achieved low LOD and visual discrimination between the three analytes using polar plots (Figure 5); however, further statistical analysis is required [99]. The same group has also developed a $3 \times 3$ sensor array based on $\mathrm{WO}_{3}, \mathrm{SnO}_{2}$, or $\mathrm{In}_{2} \mathrm{O}_{3}$-based thin films, $\mathrm{Au}$ NPs-decorated thin films and Au NP-decorated villi-like structures; the array efficiently discriminated ppt levels of $\mathrm{H}_{2} \mathrm{~S}$ and ppb levels of $\mathrm{NH}_{3}$ and $\mathrm{NO}$ in $80 \%$ of $\mathrm{RH}$, being potentially applicable to halitosis, renal disorder, and asthma diagnosis, respectively [100]. The notable sensor sensitivity was attributed to the increased surface-to-volume ratio created by the Au NPs and the highly porous villi-like structure [100].

Apart from MNPs-decorated MOS, other promising types of hybrid materials have been studied; MOS for instance have been combined with graphene derivatives. An e-Nose based on graphene-doped $\mathrm{TiO}_{2}, \mathrm{NFs}$, and nano-ribbons was recently developed for ethanol, acetone, $\mathrm{CO}$ and $\mathrm{NO}$ detection, common biomarkers for Staphylococcus aureus infection, diabetes, asthma, COPD, and cystic fibrosis (CF), respectively [101]. Breath-simulating samples of different VOCs-concentrations (respective to $\mathrm{HC}$ and patients of a selected disease) have also been studied, leading to effective discrimination of VOC concentrations. In another attempt for the development of an e-Nose, molecularly functionalized rGO layers with different amine-ligands, have been used for the discrimination of exhaled cancer biomarkers ethanol, 2-ethylhexanol, nonanal, and ethyl benzene [102]. The molecular ligands, serving as the organic sensing film, alter the adsorption capacity and conductivity of the rGO. Concerning CNTs-based cross-reactive sensor arrays, calixarene functionalization has permitted the detection of aromatic compounds (toluene, benzene, ethyl benzene, and xylenes) with high sensitivity [103]. Decoration with $\mathrm{Au}(1.5 \mathrm{~nm}), \mathrm{Pd}(0.2 \mathrm{~nm})$, and $\mathrm{Cr}$ $(1.0 \mathrm{~nm})$ has been used along with a pristine sensor element to effectively distinguish $\mathrm{NH}_{3}$, ethanol, $\mathrm{CO}$, and $\mathrm{CO}_{2}$ using PCA [104]. Remarkably, in a more recent study, an array of nylon fibers wrapped with SWCNTs, MWCNTs, and ZnO QDs-SWCNTs has been developed as a flexible wearable sensor to be incorporated in face masks. Detection and effective discrimination of $\mathrm{NH}_{3}$, ethanol, and formaldehyde [105], and common disease biomarkers (ethanol [101], formaldehyde [106], $\mathrm{NH}_{3}$ [7]) was achieved [7]. SWCNTs-functionalization with semiconducting organic polymers, monomers or oligomers is also reported, especially for real-world sample experiments, discriminating successfully COPD patients from 
HC as well as selected analytes; however, larger clinical trials remain to be conducted (Table 2) [107].

(a)

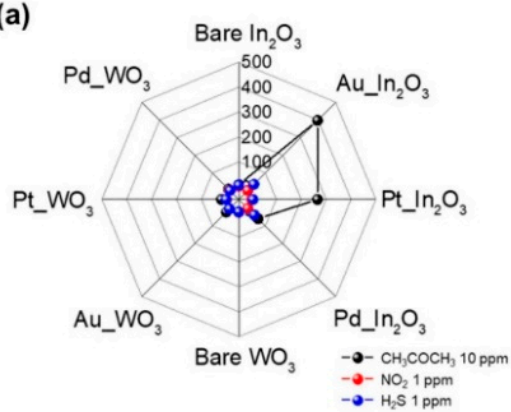

(b)

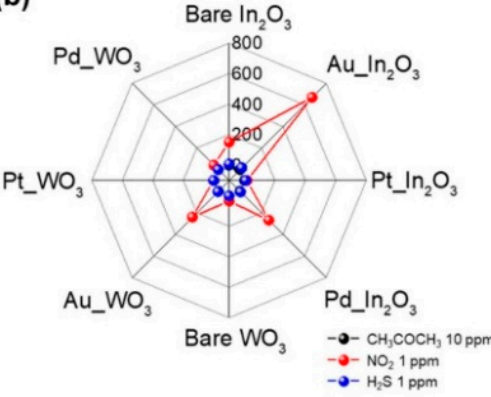

(c)

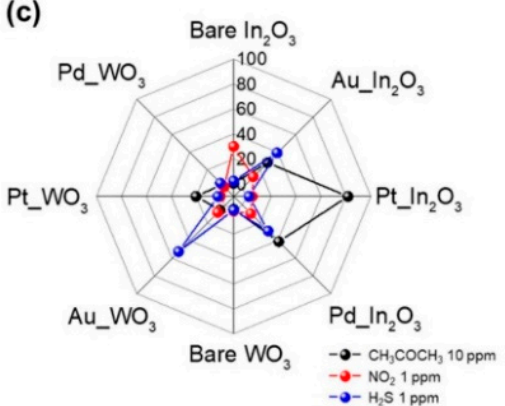

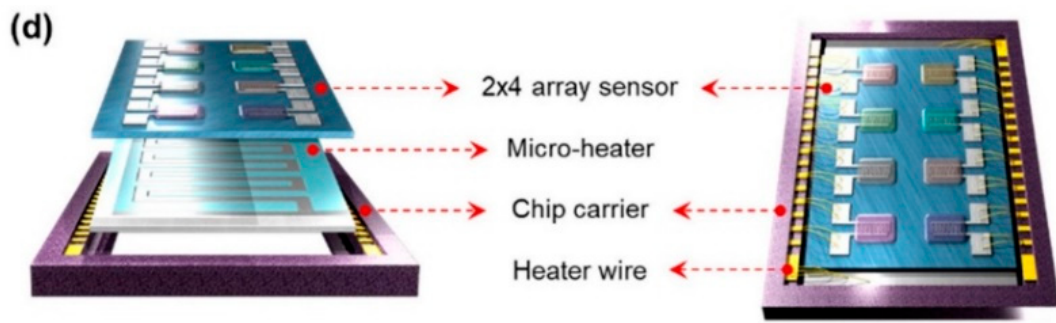

Figure 5. Polar plot of a $2 \times 4$ sensor array response for (a) $10 \mathrm{ppm}$ of acetone; (b) $1 \mathrm{ppm}$ of $\mathrm{NO}_{2}$ and (c) 1 ppm of $\mathrm{H}_{2} \mathrm{~S}$ and operation temperatures 300,150 , and $250{ }^{\circ} \mathrm{C}$; (d) schematic representation of the parts of the sensor $(2 \times 4$ sensor array, back heater, chip carrier, and Au wires). The array shows good recovery and repeatability as well as high performance at sub ppb level, facilitating discrimination between the three biomarkers. Adapted with permission from Ref. [99]. Copyright ( 2021 by the authors. Licensee MDPI, Basel, Switzerland.

The most common types of hybrid sensing layers are those composed of a conductive inorganic material, surrounded by an organic functional film where the gaseous analytes are adsorbed therefore changing the conductivity of the device [15]. CNTs, MNPs (e.g., Au) [15], or carbon black $[15,108]$ can be used as the conductive part while non-conducting molecular ligands (MCNPs) [51,108,109] or polymers [110] as the organic one. Such sensors offer the prospect of cross-reactive sensor-array development since the organic part is selected based on the chemical and physical properties of the VOCs [16]. Upon sensor exposure to gaseous biomarkers, the latter diffuse into the organic layer interacting with its functional groups [15]. Those interactions cause swelling of the organic matrix [15], posing a stress to the underlying NPs layer [41], thus increasing the inter-particle distance of the conducting NPs and the measured resistance [6]. In addition, the relative dielectric constants of the VOCs and the organic layer, all of which affect the permittivity of the organic matrix, change the measured resistance [6].

Molecularly-functionalized conducting nanomaterials have been extensively investigated for the cross-reactive detection of VOCs by Haick et al. Molecularly-capped Au NPs and molecularly-coated random SWCNTs networks have been successfully developed for exhaled VOCs-biomarkers detection using real-world samples, usually in combination with chromatographic analysis of breath samples. Irritable bowel syndrome disease (IBS) [111], ovarian [44], colon, lung, gastric [16,112], breast and prostate cancer [16,42], chronic kidney diseases (CKD) [113], multiple sclerosis [62], Alzheimer's and Parkinson's diseases [15,114] have been effectively diagnosed with such chemiresistors. In the case of multiple sclerosis e.g., PAH-coated SWCNTs sensor-arrays have exhibited sensitivity, specificity and accuracy percentages comparable to those of invasive and expensive techniques such as MRI and cerebrospinal fluid electrophoresis [62]. VOCs targeted for the diseases mentioned above are presented on Table 2. In a remarkable application of a MCNPs/SWCNTs-based chemire- 
sistor array (20 sensors), 17 different diseases (Table 2) were successfully discriminated, with $86 \%$ accuracy, based on the detection of a pattern of only 13 VOC whose concentration differed significantly between HC and/or different diseases [115]. More recently, a MCNPs-based chemiresistor was developed for COVID-19 detection, with remarkable diagnostic accuracy over healthy and non-COVID infected subjects [24]. Despite the fact that targeted VOCs were not listed in this study, the most notable VOCs for COVID-19 are probably methylpent-2-enal, 2,4-octadiene, 1-chloroheptane, and nonanal (10-250 ppb) [25]. Interestingly, a tailor-made nanoscale artificial nose (NA-NOSE), based on this type of sensing films, has been developed by Haick et al. [116]. NA-NOSE is composed of seven cross-reactive MCNPs-layers with different ligands, from which 6 are composed of spherical Au NPs and 1 of cubic Pt NPs. NA-NOSE seems to be promising for breath analysis, as it has been effectively used, e.g., for BC diagnosis [116]. Molecularly-modified $\mathrm{Au}$, Pt, and $\mathrm{Cu}$ NPs have been used for the development of a 6-sensors array, to effectively diagnose the infectious disease human cutaneous Leishmaniasis with a $98.2 \%$ accuracy, after identifying 9 potential biomarkers using GC/quadruple-TOF [109]. A representative example of MCNPs-based chemiresistors is presented in Figure 6.

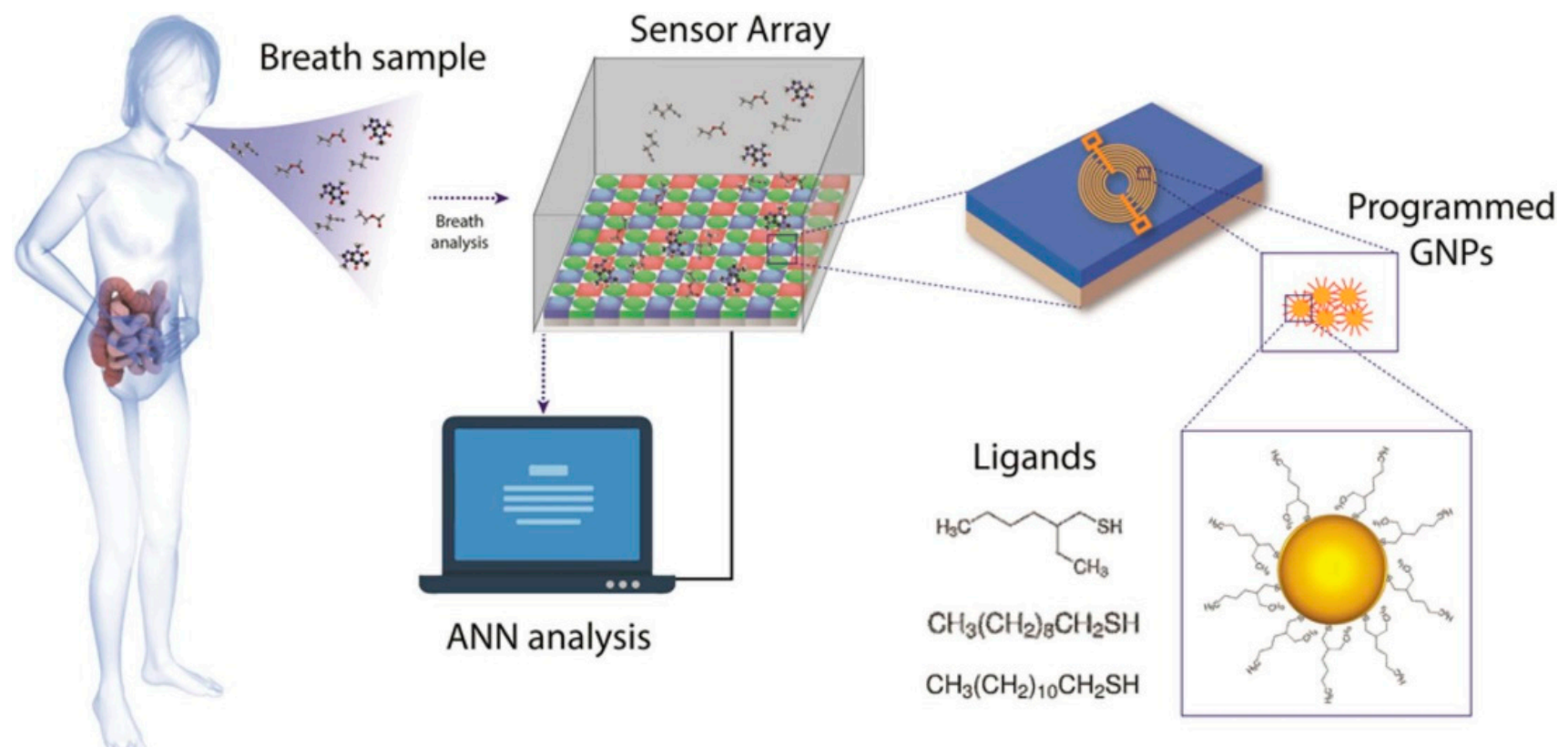

Figure 6. Schematic representation of a MCNPs-based chemiresistor, programmed according to the VOCs identified as inflammatory bowel and irritable bowel syndrome disease biomarkers. The sensor was exposed to simulated, HC and patient breath samples. Time-dependent and reversible shift in the sensor's resistance is associated with the MCNPs-VOCs interactions. The variability of molecular ligands leads to varying sensing responses; pattern recognition methods such as ANN, permit the development of effective diagnostic classifiers. Reprinted with permission from ref. [111]. Copyright (C) 2016 WILEY-VCH Verlag GmbH \& Co. KgaA, Weinheim.

The size, composition, inter-particle distance, and periodicity of the NPs as well as the aggregate thermal stability of such sensors are essential yet easily controlled parameters [51]. However, one major drawback is humidity cross-sensitivity which is a major component of exhaled breath. Thus, sensor reliability and reproducibility for real samples analysis is of major concern. Humidity compensation is proposed as an effective solution, enhancing the diagnostic ability of the sensor [42].

Remarkably, hybrid composites based on polymers seem particularly promising as well. One of the most commonly studied commercial e-Noses i.e., Cyranose 320, consists of 32 chemiresistors based on composites of carbon black conducting particles and different polymeric films $[117,118]$. Cyranose 320 has been effectively used amongst others, for the 
discrimination of asthma [119], COPD [120], BC [121], or pneumonia [122] patients from HC. Among self-developed sensor arrays, CNTs combined with 5 different polymers have permitted the effective differentiation of 9 VOCs as LC potential biomarkers using PCA analysis (Table 2) [110]. Notably, the combinations of MNPs with polymers, apart from molecular organic ligands, comprise a promising type of coating materials for chemical cross-selectivity to be achieved. Chemically unmodified MNPs (Pt [48,123] or Au [71] of mean diameter 4-5 nm [41,48,123]), coated with different polymeric films and with affinity for different compounds, have been investigated along with pattern recognition methods for pesticide detection [41] and more recently pesticide discrimination (chlorpyrifos, bupirimate, and humidity) [124]. The sensing film is deposited on Au IDEs [41] while the device can be fabricated on rigid (e.g., oxidized Si wafer) [41,48] or flexible (e.g., polyimide) [123] substrate as can be seen in Figure 7. Such sensors could be particularly promising for disease diagnosis applications, as semi-selective sensors of exhaled VOCs.
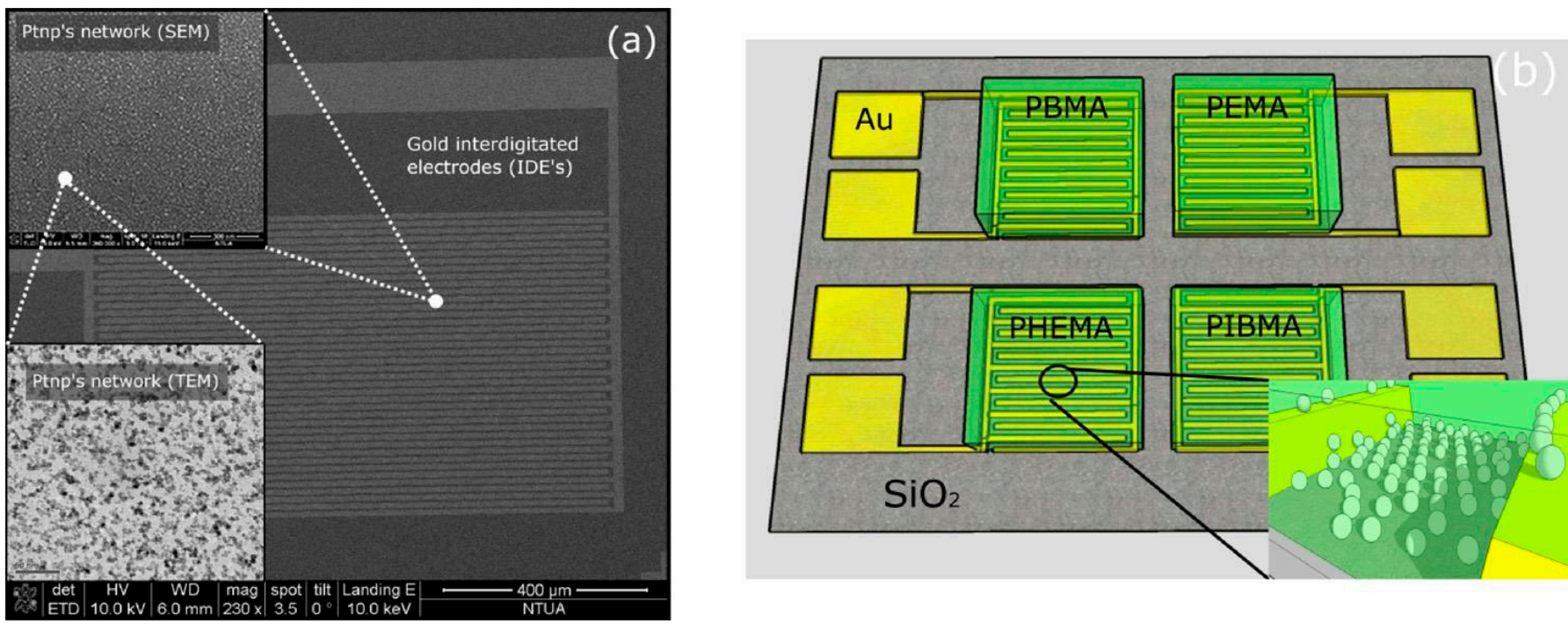

Figure 7. (a) SEM image of an array of polymer-coated PtNPs-based chemiresistors, developed for pesticide detection, TEM image of PtNPs layer, with surface coverage $46 \%$ and mean nanoparticle diameter of $5 \mathrm{~nm}$; (b) schematic representation of the sensor array. Different polymer susceptibility towards water/pesticide vapors leads to a gas-sensing array that is capable of identifying each of the gas-analytes. Reprinted with permission from Ref. [41] Copyright (C) 2018 Elsevier Ltd. 
Table 2. Sensor arrays for exhaled VOCs detection as biomarkers of several diseases, in real-world or synthetic samples, using conventional materials and/ or nanomaterials.

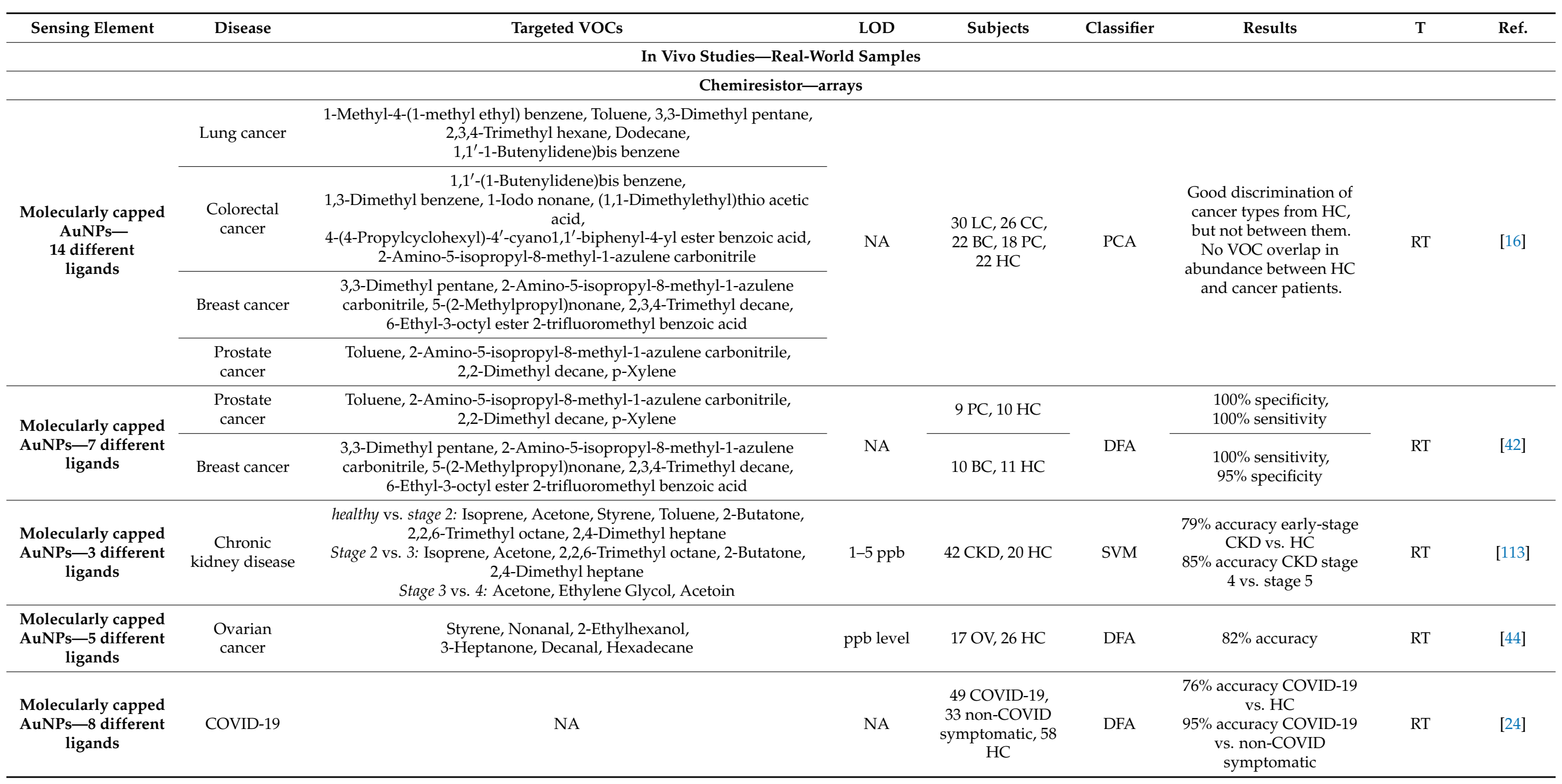


Table 2. Cont.

\begin{tabular}{|c|c|c|c|c|c|c|c|c|}
\hline Sensing Element & Disease & Targeted VOCs & LOD & Subjects & Classifier & Results & $\mathbf{T}$ & Ref. \\
\hline $\begin{array}{l}\text { PAH-coated random } \\
\text { SWCNTs network- } \\
4 \text { different } \\
\text { PAHs }\end{array}$ & $\begin{array}{l}\text { Multiple } \\
\text { sclerosis }\end{array}$ & Hexanal, 5-Methyl-undecane & NA & $37 \mathrm{MS}, 18 \mathrm{HC}$ & DFA & $80.4 \%$ accuracy & RT & [62] \\
\hline $\begin{array}{l}\text { Molecularly caped } \\
\text { AuNPs/CDs-coated } \\
\text { random SWCNTs } \\
\text { network- } \\
20 \text { different sensing } \\
\text { films }\end{array}$ & $\begin{array}{l}\text { Alzheimer's } \\
\text { and } \\
\text { Parkinson's } \\
\text { disease }\end{array}$ & $24 \mathrm{VOCs}$ & $1-5 \mathrm{ppb}$ & $\begin{array}{l}15 \mathrm{AD}, 30 \mathrm{PD} \\
12 \mathrm{HC}\end{array}$ & DFA & $\begin{array}{l}85 \% \text { accuracy } A D \text { vs. } \mathrm{HC} \\
78 \% \text { accuracy } P D \text { vs. } \mathrm{HC} \\
84 \% \text { accuracy } A D \text { vs. PD }\end{array}$ & RT & [114] \\
\hline $\begin{array}{l}\text { Molecularly caped } \\
\text { AuNPs/PAH-coated } \\
\text { random SWCNTs } \\
\text { network- } \\
20 \text { different sensing } \\
\text { films }\end{array}$ & $\begin{array}{l}17 \text { diseases } \\
\text { (LC, CC, HNC, } \\
\text { OC, BLC, PC, } \\
\text { KC, GC, CD, } \\
\text { UC, IBS, IPD, } \\
\text { MS, PDISM, } \\
\text { PH, PET, } \\
\text { CKD) }\end{array}$ & $\begin{array}{l}\text { 2-Ethylhexanol, 3-Methylhexane, } \\
\text { 5-Ethyl-3-methyloctane, Acetone, Ethanol, Ethyl acetate, Ethyl benzene, } \\
\text { Isononane, Isoprene, Nonanal, Styrene, Toluene, Undecane }\end{array}$ & $10 \mathrm{ppb}$ & $\begin{array}{l}813 \text { any of } \\
17 \text { diseases, } \\
591 \text { HC }\end{array}$ & $\begin{array}{l}\text { DFA, } \\
\text { HCA }\end{array}$ & $86 \%$ average accuracy & RT & [115] \\
\hline $\begin{array}{l}\text { Ligand capped Au, } \\
\text { Pt, and } \\
\text { CuNPs-6 different } \\
\text { sensing films }\end{array}$ & $\begin{array}{l}\text { Human } \\
\text { cutaneous } \\
\text { leishmaniasis }\end{array}$ & $\begin{array}{l}\text { 2,2,4-trimethyl pentane, 4-methyl-2-ethyl-1-pentanol, methyl vinyl } \\
\text { ketone, nonane, 2,3,5-trimethyl hexane, } \\
\text { hydroxy-2,4,6-trimethyl-5-(3-methyl-2 butenyl)cyclohexyl) methyl } \\
\text { acetate, 3-ethyl-3-methyl heptane, octane, } \\
\text { 2-methyl-6-methylene-octa-1,7-dien-3-ol }\end{array}$ & NA & $28 \mathrm{HCL}, 32 \mathrm{HC}$ & PCA, DFA & $\begin{array}{l}98.2 \% \text { accuracy, } 96.4 \% \\
\text { sensitivity, } 100 \% \\
\text { specificity }\end{array}$ & RT & [109] \\
\hline $\begin{array}{l}\text { pristine, COOH-, } \\
\text { Hex-4T- } \\
\text { Hex/DNA/oligomers, } \\
\text { PTCDA/TAPC/TCTA } \\
\text { monomers or PANI- } \\
\text { functionalized } \\
\text { SWCNTs }\end{array}$ & COPD & $\begin{array}{c}\mathrm{NH}_{3}, \mathrm{NO}_{2}, \mathrm{H}_{2} \mathrm{~S} \text {, benzene, 2-propanol, acetone, ethanol, sodium } \\
\text { hypochlorite, water }\end{array}$ & sub-ppb & $12 \mathrm{COPD}, 9 \mathrm{HC}$ & PCA & $\begin{array}{l}\text { Acetone, ethanol and } \\
\text { 2-propanol selective } \\
\text { PANI-, TAPC- and } \\
\text { COOH-CNTs, } \\
\text { respectively. } \\
\mathrm{NO}_{2} \text { relevant driver of } \\
\text { real-samples } \\
\text { classification. } \\
\text { Larger clinical trials } \\
\text { needed. }\end{array}$ & RT & [107] \\
\hline $\begin{array}{c}\text { Pristine } \mathrm{WO}_{3} \\
0.008 \mathrm{wt} \% \\
\text { and } 0.042 \mathrm{wt} \% \\
\text { Pt-WO } \\
\text { macroporous NFs }\end{array}$ & Halitosis & $\begin{array}{l}\mathrm{H}_{2} \mathrm{~S} \text { and Methyl mercaptan } \\
\text { (in presence of Toluene and Acetone) }\end{array}$ & sub-ppm & $\begin{array}{l}4 \text { simulated } \\
\text { halitosis breath } \\
\text { samples (1 ppm), } \\
\quad 4 \mathrm{HC}\end{array}$ & PCA & Successful classification & $350^{\circ} \mathrm{C}$ & [96] \\
\hline
\end{tabular}


Table 2. Cont.

\begin{tabular}{|c|c|c|c|c|c|c|c|c|}
\hline Sensing Element & Disease & Targeted VOCs & LOD & Subjects & Classifier & Results & $T$ & Ref. \\
\hline $\begin{array}{l}7 \text { different } \\
\text { commercial MOS }\end{array}$ & Lung cancer & $\begin{array}{l}\text { Ethyl benzene, 4-Methyl octane, } \\
\text { Undecane, 2,3,4-trimethyl hexane }\end{array}$ & $\begin{array}{l}\text { Down to a } \\
\text { few ppb }\end{array}$ & $\begin{array}{c}37 \text { NSCLC } \\
(81.1 \% \text { I, II), } \\
48 \mathrm{HC}\end{array}$ & PCA & $\begin{array}{l}75 \% \text { accuracy } \\
\text { Promising prognostic } \\
\text { tool after LC resection } \\
\text { surgery }\end{array}$ & $300^{\circ} \mathrm{C}$ & [89] \\
\hline $\begin{array}{c}5 \text { different } \\
\text { commercial MOS }\end{array}$ & $\begin{array}{l}\text { Lung cancer, } \\
\text { COPD }\end{array}$ & NA & NA & $\begin{array}{l}32 \mathrm{LC}, 38 \mathrm{COPD} \\
72 \mathrm{HC}\end{array}$ & $\begin{array}{l}\text { PCA, } \\
\text { SVM, } \\
\text { k-nearest } \\
\text { neighbors }\end{array}$ & $\begin{array}{c}\text { LC vs. HC: } 91.3 \% \\
\text { accuracy, } 84.4 \% \\
\text { sensitivity and } 94.4 \% \\
\text { specificity } \\
\text { COPD vs. HC: } 90.9 \% \\
\text { accuracy, } 81.6 \% \\
\text { sensitivity and } 95.8 \% \\
\text { specificity }\end{array}$ & NA & [87] \\
\hline \multicolumn{9}{|c|}{ Field Effect Transistor (FET)—arrays } \\
\hline $\begin{array}{c}\text { Molecularly } \\
\text { modified SiNWs }\end{array}$ & Gastric cancer & $\begin{array}{l}\text { 2-Propenenitril, Furfural, } \\
\text { 6-Methyl-5-heptene-2-one }\end{array}$ & $\begin{array}{l}\text { Down to a } \\
\text { few ppb }\end{array}$ & $30 \mathrm{GC}, 77 \mathrm{HC}$ & DFA & $>85 \%$ accuracy & RT & [125] \\
\hline \multirow[b]{2}{*}{$\begin{array}{l}\text { Molecularly } \\
\text { modified SiNWs }\end{array}$} & Gastric cancer & $\begin{array}{l}\text { 2-Propenenitril, Furfural, } \\
\text { 6-Methyl-5-heptene-2-one }\end{array}$ & \multirow{2}{*}{$\begin{array}{l}\text { Down to a } \\
\text { few ppb }\end{array}$} & \multirow{2}{*}{$\begin{array}{c}149 \text { LC, } 40 \text { GC, } \\
56 \text { Asthma/COPD, } \\
129 \text { HC }\end{array}$} & \multirow[b]{2}{*}{$\begin{array}{l}\text { DFA, } \\
\text { ANN }\end{array}$} & \multirow[b]{2}{*}{$>80 \%$ accuracy } & \multirow[b]{2}{*}{ RT } & \multirow[b]{2}{*}{ [43] } \\
\hline & Lung cancer & $\begin{array}{c}\text { Heptane, Decane, 2-Methyl pentane, } \\
\text { 2-Ethyl-1-hexanol, Propanal, Pentanal, Acetone }\end{array}$ & & & & & & \\
\hline \multicolumn{9}{|c|}{ Electrochemical sensor } \\
\hline \multirow[b]{2}{*}{$\begin{array}{c}\text { Commercial NO, } \\
\text { CO sensors, carbon } \\
\text { electrode with } \\
\text { linear-aldehyde } \\
\text { selective porous } \\
\text { poly } \\
\text { tetrafluoroethylene } \\
\text { membrane }\end{array}$} & Diabetes & & & $\begin{array}{l}15 \text { diabetic, } \\
14 \mathrm{HC}\end{array}$ & & \multirow{2}{*}{$\begin{array}{l}\text { LC vs. HC, diabetic vs. HC } \\
\text { Cross-sensitivity for } \\
\text { aldehyde sensor: } \\
\text { Moderate for high level } \\
\text { of ethanol and } \\
\text { isopropanol/Weak for } \\
\mathrm{H}_{2} \mathrm{~S}, \mathrm{NO} \text {, methanol, } \\
\text { 3-heptanone/None for } \\
\mathrm{NO}_{2} \text {, propofol, isoprene, } \\
\text { or acetone }\end{array}$} & \multirow[b]{2}{*}{ RT } & \multirow[b]{2}{*}{ [126] } \\
\hline & Lung cancer & $\begin{array}{c}\text { NO, CO, Formaldehyde, Acrolein, Propanal, Crotonaldehyde, Butanal, } \\
\text { Pentanal, Hexanal, Heptanal, Octanal, Nonanal, Decanal, } \\
\text { Acetaldehyde }\end{array}$ & Low $\mathrm{ppb}$ & $\begin{array}{l}3 \mathrm{LC}, 3 \text { smokers, } \\
\quad 3 \mathrm{HC}\end{array}$ & & & & \\
\hline
\end{tabular}


Table 2. Cont.

\begin{tabular}{|c|c|c|c|c|c|c|c|c|}
\hline Sensing Element & Disease & Targeted VOCs & LOD & Subjects & Classifier & Results & $\mathbf{T}$ & Ref. \\
\hline \multicolumn{9}{|c|}{ Optical-Colorimetric sensor arrays } \\
\hline $\begin{array}{l}24 \text { chemically } \\
\text { reactive colorants }\end{array}$ & Lung cancer & NA & Low ppm & $92 \mathrm{LC}, 137 \mathrm{HC}$ & LPM & $\begin{array}{c}\text { Accuracy } 81.1 \% \text { LC vs. } \\
\text { HC, } \\
\text { 82.5-89\% one histology } \\
\text { vs. HC, } \\
\text { 86.4\% ADC vs. SCC }\end{array}$ & RT & [127] \\
\hline \multicolumn{9}{|c|}{ Optical sensors } \\
\hline \multirow{3}{*}{$\begin{array}{l}\text { PMTFP-coated } \\
\text { optical fiber }\end{array}$} & $\begin{array}{c}\text { Vit. E } \\
\text { deficiency }\end{array}$ & Ethane & \multirow{3}{*}{$\mathrm{pmol} / \mathrm{L}$} & \multirow{3}{*}{$20 \mathrm{HC}$} & \multirow{3}{*}{ NA } & \multirow{3}{*}{ NA } & \multirow{3}{*}{ RT } & \multirow{3}{*}{ [128] } \\
\hline & $\begin{array}{c}\text { Liver diseases, } \\
\text { Schizophrenia, } \\
\text { Breast cancer, } \\
\text { Rheumatoid } \\
\text { Arthritis }\end{array}$ & Pentane & & & & & & \\
\hline & Lung cancer & Heptane, Octane, Decane, Benzene, Toluene, Styrene & & & & & & \\
\hline \multicolumn{9}{|c|}{$\begin{array}{l}\text { Piezoelectric (SAW) sensor arrays } \\
\end{array}$} \\
\hline $\begin{array}{c}\text { GC-column/ } \\
\text { Polyisobutylene- } \\
\text { coated SAW, } \\
\text { non-coated SAW } \\
\text { sensors } \\
\end{array}$ & Lung cancer & $\begin{array}{c}\text { Styrene, Decane, Isoprene, Benzene, Undecane, 1-Hexene, Hexanal, } \\
\text { Propyl benzene, Heptanal, 1,2,4-Trimethyl benzene, Methyl } \\
\text { cyclopentane }\end{array}$ & $500 \mathrm{ppb}$ & $\begin{array}{l}20 \mathrm{LC}, 15 \mathrm{HC} \text {, } \\
7 \text { chronic } \\
\text { bronchitis }\end{array}$ & ANN & $\begin{array}{c}80 \% \text { sensitivity and } \\
\text { specificity }\end{array}$ & RT & {$[6,129]$} \\
\hline \multicolumn{9}{|c|}{ Piezoelectric $(\mathrm{QCM})$ sensor arrays } \\
\hline $\begin{array}{c}7 \text { different } \\
\text { metalloporphyrins }\end{array}$ & COPD & NA & NA & $\begin{array}{l}5 \text { COPD per } \\
\text { GOLD stage (20), } \\
5 \text { HC }\end{array}$ & PLS-DA & $\begin{array}{c}\text { Fair repeatability of } \\
\text { measurements within } \\
\text { HC and hypoxemic } \\
\text { COPD patients (stage 4) } \\
\text { Potential COPD severity } \\
\text { assessment }\end{array}$ & $\mathrm{RT}$ & [130] \\
\hline $\begin{array}{c}8 \text { different } \\
\text { metalloporphyrins }\end{array}$ & Asthma & NA & NA & $\begin{array}{c}27 \text { asthma, } 24 \\
\text { HC }\end{array}$ & PCA, FNN & $87.5 \%$ accuracy & RT & [131] \\
\hline $\begin{array}{c}8 \text { different } \\
\text { metalloporphyrins }\end{array}$ & Lung cancer & NA & NA & $20 \mathrm{LC}, 10 \mathrm{HC}$ & PLS-DA & $\begin{array}{l}85 \% \text { accuracy LC vs. HC } \\
75 \% \text { accuracy ADC vs. } \\
\text { SCC }\end{array}$ & $\mathrm{RT}$ & [132] \\
\hline
\end{tabular}


Table 2. Cont.

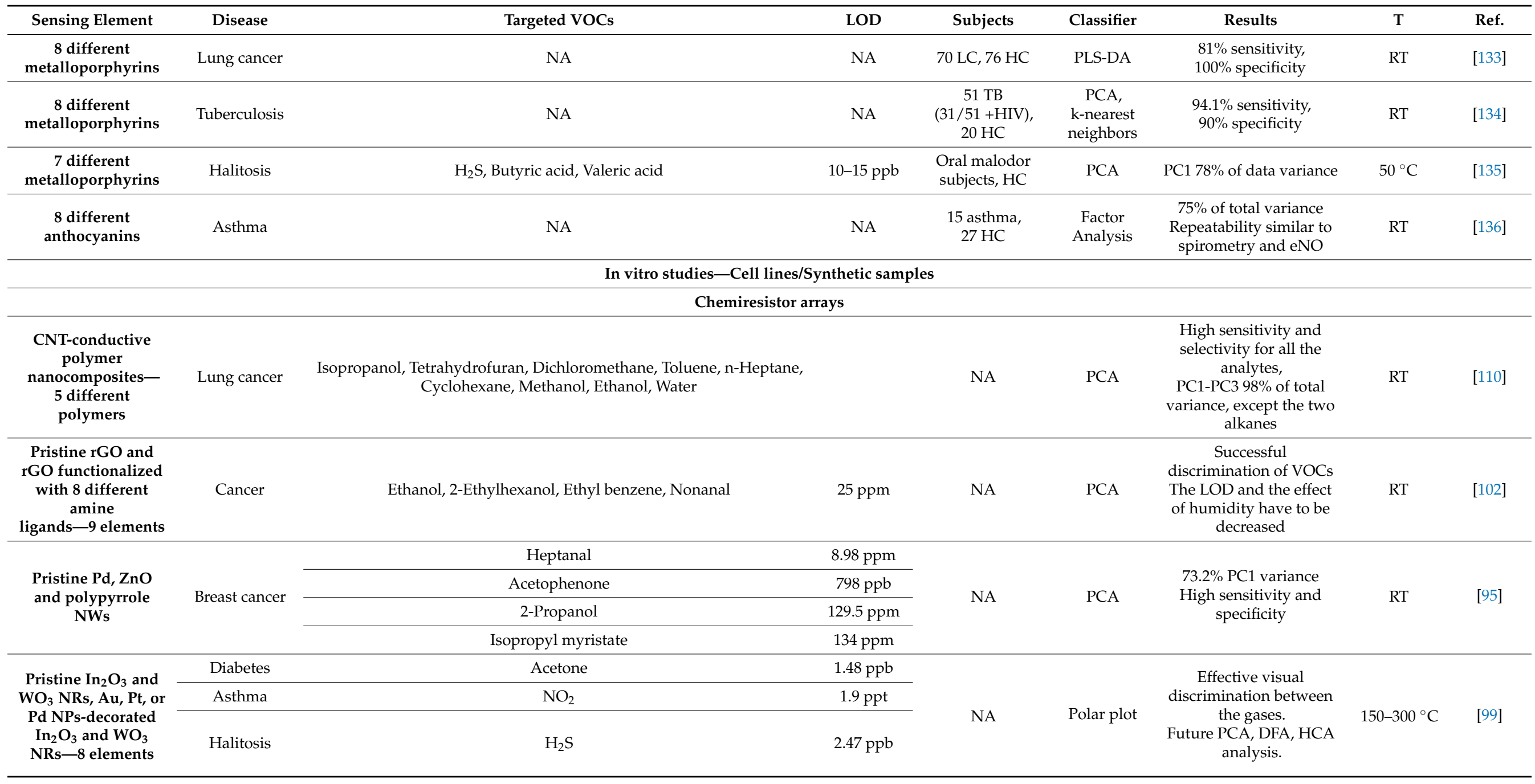


Table 2. Cont.

\begin{tabular}{|c|c|c|c|c|c|c|c|c|}
\hline Sensing Element & Disease & Targeted VOCs & LOD & Subjects & Classifier & Results & $\mathbf{T}$ & Ref. \\
\hline \multirow{2}{*}{$\begin{array}{c}\mathrm{WO}_{3} \mathrm{NTs} \\
\mathrm{Pt} \mathrm{NPs}-\mathrm{WO}_{3} \mathrm{NTs} \\
\text { Pd NPs-WO }\end{array}$} & Asthma & NO & $50 \mathrm{ppb}$ & \multirow[b]{2}{*}{ NA } & \multirow[b]{2}{*}{ NA } & \multirow[b]{2}{*}{ NA } & $350^{\circ} \mathrm{C}$ & \multirow[b]{2}{*}{ [56] } \\
\hline & Lung cancer & Toluene & $100 \mathrm{ppb}$ & & & & $400^{\circ} \mathrm{C}$ & \\
\hline \multirow{2}{*}{$\begin{array}{c}\text { Pristine, } 0.1 \% \mathrm{wt} \\
\text { GO- and } 0.1 \mathrm{wt} \% \\
\text { thin layered } \\
\text { graphite } \mathrm{WO}_{3} \mathrm{Hemi} \\
\text { tubes }\end{array}$} & Diabetes & Acetone & \multirow[b]{2}{*}{$1 \mathrm{ppm}$} & \multirow[b]{2}{*}{ NA } & \multirow[b]{2}{*}{ NA } & \multirow[b]{2}{*}{ NA } & \multirow[b]{2}{*}{$350^{\circ} \mathrm{C}$} & \multirow[b]{2}{*}{ [57] } \\
\hline & Halitosis & $\mathrm{H}_{2} \mathrm{~S}$ & & & & & & \\
\hline \multicolumn{9}{|c|}{ Electrochemical sensor } \\
\hline \multirow{2}{*}{$\begin{array}{l}\text { MWCNTs/Au-Ag } \\
\text { NPs/GCE }\end{array}$} & \multirow{2}{*}{ Gastric cancer } & 3-Octanone & $0.3 \mathrm{ppb}$ & \multirow{2}{*}{$\begin{array}{l}\text { MGC-803 GC } \\
\text { and GES-1 } \\
\text { gastric mucosa } \\
\text { cell lines }\end{array}$} & \multirow{2}{*}{ NA } & \multirow{2}{*}{$\begin{array}{c}\text { Easy cell line } \\
\text { discrimination, high } \\
\text { sensitivity, good VOCs } \\
\text { selectivity in presence of } \\
\mathrm{CO}_{2} \text {, acetone and ethanol }\end{array}$} & \multirow{2}{*}{ RT } & \multirow{2}{*}{ [137] } \\
\hline & & Butanone & $0.5 \mathrm{ppb}$ & & & & & \\
\hline SiNWs-rGO & $\begin{array}{l}\text { Infectious } \\
\text { diseases }\end{array}$ & $\begin{array}{l}\text { Cyclohexane, Formaldehyde in presence of Methanol, Ethanol, } \\
\text { Acetonitrile, Acetaldehyde and humidity }\end{array}$ & $1 \mathrm{ppm}$ & NA & & $\begin{array}{l}\text { Novel electrode platform } \\
\text { with increased sensitivity, } \\
\text { selectivity and } \\
\text { repeatability }\end{array}$ & & [106] \\
\hline \multicolumn{9}{|c|}{ Piezoelectric (SAW) sensor arrays } \\
\hline $\begin{array}{c}\text { SH-Calix4arene, } \\
\text { AuNRs, AgNCs, } \\
\text { Calix4arene- } \\
\text { AuNRs, } \\
\text { Calix4arene-AgNCs }\end{array}$ & Lung cancer & Chloroform, Toluene, Isoprene, Acetone, n-Hexane, Ethanol & $\begin{array}{l}1.52-12.34 \mathrm{ppm} \\
\text { for } \mathrm{CHCl}_{3} \\
1.54-2.64 \mathrm{ppm} \\
\text { for toluene }\end{array}$ & NA & NA & $\begin{array}{c}\text { Sensitivity } \uparrow \text { for all VOCs } \\
\text { Chloroform, toluene: } 6-8 \\
\text { times higher sensitivity } \\
\text { than individual } \\
\text { responses } \\
\text { Selectivity } \uparrow: \\
\text { modified AuNRs for } \\
\text { CHCl } \\
\text { modified } \mathrm{AgNCs}^{-} \text {for } \\
\text { Toluene }\end{array}$ & RT & [49] \\
\hline $\begin{array}{l}\text { Pristine or AuNPs- } \\
\text { functionalized } \\
\text { zeolitic-imidazole- } \\
\text { framework } \\
\text { nanocrystals (ZIF-8, } \\
\text { ZIF-67) }\end{array}$ & Diabetes & Acetone, Ammonia, Ethanol & $\begin{array}{c}\text { acetone } \\
1.1-3.6 \mathrm{ppm}, \\
\text { ethanol } \\
0.5-3 \mathrm{ppm} \\
\mathrm{NH}_{3} \\
1.6-3.2 \mathrm{ppm}\end{array}$ & NA & PCA & $\begin{array}{l}\text { Effective discrimination } \\
\text { of diabetes biomarkers }\end{array}$ & RT & [138] \\
\hline
\end{tabular}


Table 2. Cont.

\begin{tabular}{|c|c|c|c|c|c|c|c|c|}
\hline Sensing Element & Disease & Targeted VOCs & LOD & Subjects & Classifier & Results & $\mathbf{T}$ & Ref. \\
\hline \multicolumn{9}{|c|}{ Piezoelectric (QCM) sensor arrays } \\
\hline \multirow{2}{*}{$\begin{array}{c}\mathrm{TiO}_{2}-\mathrm{MWCNTS} \\
\text { and Cobalt (II) } \\
\text { phthalocyanine- } \\
\text { silica on } \mathrm{Au} \\
\text { layers } \\
\end{array}$} & Diabetes & Acetone & $4.33 \mathrm{ppm}$ & & & & & \\
\hline & Asthma & $\mathrm{NO}$ & $5.75 \mathrm{ppb}$ & NA & NA & High sensitivity & RT & [139] \\
\hline \multicolumn{9}{|c|}{ Optical-Colorimetric arrays } \\
\hline $\begin{array}{l}36 \text { chemically } \\
\text { responsive dyes } \\
\text { (porphyrin } \\
\text { derivatives, } \\
\text { NaFluo) }\end{array}$ & Lung cancer & p-Xylene, Styrene, Isoprene, Hexanal & $50 \mathrm{ppb}$ & NA & $\begin{array}{c}\text { HCA, } \\
\text { PCA, } \\
\text { BPNN }\end{array}$ & $\begin{array}{l}100 \% \text { accuracy of kind } \\
\text { and concentration } \\
\text { discrimination, } \\
\text { promising for } \\
\text { real-sample experiments }\end{array}$ & RT & [140] \\
\hline $\begin{array}{l}\text { AuNRs-modified } \\
\text { metalloporphyrins } \\
\text { and } \mathrm{pH} \text { responsive } \\
\text { dyes-36 spots }\end{array}$ & Lung cancer & $\begin{array}{c}\text { Decane, Undecane, Hexanal, Heptanal, 1,2,4-Trimethylbenzene, } \\
\text { Benzene }\end{array}$ & $<1 \mathrm{ppm}$ & NA & $\begin{array}{l}\text { PCA, } \\
\text { HCA }\end{array}$ & $\begin{array}{c}64.2 \% \text { accuracy of } \\
\text { structurally similar } \\
\text { VOCs, } 93 \% \\
\text { photoprotection of } \\
\text { metalloporphyrins, } \uparrow \\
\text { repeatability and } \\
\text { long-term stability }\end{array}$ & RT & [141] \\
\hline
\end{tabular}

AD: Alzheimer's Disease, ADC: Adenocarcinoma, ANN: Artificial Neural Network, BC: Breast cancer, BLC: Bladder cancer, BPNN: Back-Propagation Neural Network, BUN: Blood urea nitrogen, CC: Colorectal cancer, CD: Crohn's Disease, CDs: Cyclodextrin derivatives, CKD: Chronic Kidney Disease, COPD: Chronic Obstructive Pulmonary Disease, DFA: Discriminant Function Analysis, FNN: Feet-forward Neural Network, GC: Gastric cancer, GCE: Glass Carbon Electrode, GOLD: Global Initiative for Obstructive Lung Disease, HC: Healthy control, HCA: Hierarchical Cluster Analysis, HNC: Head and Neck cancer, IBS: Irritable bowel syndrome, IPD: Idiopathic Parkinson's disease, KC: Kidney cancer, LC: Lung cancer, LOD: Limit of Detection, LPM: Logistic prediction model, MLP: Multi-layer Perceptron, MS: Multiple Sclerosis, MWCNTs: Multi-wall carbon nanotubes, NA: Not Applicable, NaFluo: sodium fluorescein, NCs: Nanocubes, NFs: Nanofibers, NPs: Nanoparticles, NRs: Nanorods, NSCLC: Non-small Cell Lung Carcinoma, OC: Ovarian cancer, PAH: Polycyclic aromatic hydrocarbons, PC: Prostate cancer, PCA: Principal Component Analysis, PD: Parkinson's Disease, PDISM: Atypical Parkinsonism, PET: Pre-eclampsia toxemia, PH: Pulmonary Hypertension, PLS-DA: Partial Least Square Discriminant Analysis, PMTFP: Polymethyl(3,3,3-trifluoropropyl)siloxane, RT: Room temperature, SCC: Squamous cell carcinoma, SiNWs: Silicon nanowires, SVM: Support Vector Machine, T: Temperature, TB: Tuberculosis, TFB: Poly(9,9-dioctylfluorenyl-2,7-diyl)-co-(4,4'-(N-(4-s-butylphenyl)diphenylamine), UC: Ulcerative Colitis. 


\subsection{Field-Effect Transistors (FET)}

Field-effect transistors are voltage-controlled [6] devices consisting of two electrodes (the source and the drain electrode), a semiconducting channel, an insulating gate, and a conducting gate electrode [13]. The current flows between the source terminal and the drain terminal through the semiconducting channel by applying a source-drain potential. Voltage applied between the source terminal and the gate terminal controls the current flow between source and drain as well as the conductivity of the conduction channel. For a constant source-gate voltage, exposure to gaseous analytes can affect the conductivity of the conduction channel $[6,13]$. Notably, the main difference between chemiresistors and FET sensors is the ability of the latter to provide not only current variations but also threshold voltage changes upon analyte exposure [142].

Depending on the gaseous analyte-type and the carrier type of the channel material (holes/electrons), the charge carrier concentration of the semiconducting channel material can be changed upon device exposure. In the case of an n-channel FET, oxidizing gas exposure reduces the majority carriers of the channel region thus decreasing the current flow. In the case of exposure to a reducing gas or of a FET with a p-type channel exposed to an oxidizing gas, the current flowing through the channel increases. Measurement of current variations permits the detection as well as the quantification of analytes after appropriate calibration [13]. The applied gate voltage permits control of sensitivity, providing it is set so as to permit the maximum conductance variation [142].

A chemical FET can also possess a gas-selective coating/a series of coatings, between the transistor gate and the analyte. Different chemical modification of the gate allows reaction with different chemical species hence permitting their differentiation $[43,143]$. For ion sensitive FETs charged species at the sensing interface of the gate, change the polarization of the underlying semiconductor/dielectric interface. Electron conductance through the semiconducting channel is sensitive to gate polarization and the chemical modification of the gate can either attract or repel the semiconductor-charge carriers. Thus, by measuring the source-drain current, the polarization of the sensitive interface is determined [51].

Channel conductivity is also affected by the gas-analyte polarity. Adsorption (molecular gating) of polar molecules on the outer surface of the conducting channel is considered to provoke changes in the electric field. Molecular binding of non-polar molecules can potentially change the density of charged surface states of the functionalized semiconductor surface, due to analyte induced conformational alternations, or can affect the dielectric medium close to the semiconductor surface $[6,144]$.

\subsubsection{Selective Applications}

Selective FET sensors are commonly developed using nanomaterials for the formation of the conductive channel and used mainly for the detection of simple gases. CNTs (specifically SWCNTs acting as p-semiconductors) have been extensively investigated for CNTFET gas sensors, possessing p-transistor characteristics $[7,50]$. Pristine CNTs have been used for the detection of the oxidizing gases $\mathrm{NO}$ [145] and $\mathrm{NO}_{2}$ [145-147] and the reducing gas $\mathrm{NH}_{3}[145,147]$, as well as for ethanol and benzene detection [147]. Remarkably, the CNTFET sensor developed by Chang et al. exhibited the ability to discriminate the gaseous analytes due to distinguishable temporal response of conductance to gate voltage pulse [147].

2D material-based FETs have been also developed using TMDs or graphene derivatives. $\mathrm{WS}_{2}$ n-type semiconducting multilayer nano-flakes have been used for ethanol and $\mathrm{NH}_{3}$ detection under illumination [148], while $\mathrm{MoS}_{2}$ multilayer-based FETs permit NO [149], $\mathrm{NH}_{3}$, and $\mathrm{NO}_{2}$ detection [150]. rGO-based FETs have also permitted ethanol [151], $\mathrm{NH}_{3}$ and $\mathrm{NO}_{2}$ detection achieving low ppb LOD [152], while $\mathrm{NH}_{3}$ detection was also reported using $\mathrm{NO}_{2}$-dopped graphene [153].

Hybrid materials have also been used in selective FET sensors. 2D graphene/ $\mathrm{MoS}_{2}$ heterostructures used in flexible and potentially wearable p-type devices, in which graphene 
replaces the metallic electrodes, have been reported for $\mathrm{NO}_{2}$ and $\mathrm{NH}_{3}$ detection albeit with lower sensitivity [154]. Combinations of CNTs with other materials for sensing performance improvement are also reported in the literature. Polypyrrole-SWCNTs have been used for $\mathrm{NH}_{3}$ detection, achieving increased sensitivity and reduced response/recovery times by controlling film thickness, SWCNTs concentration and annealing temperature [155]. Selective $\mathrm{H}_{2} \mathrm{~S}$ detection has been also attempted using Au-decorated SWCNTs, leading to high Au NPs size-dependent sensitivity and lower LOD ( $\leq 100 \mathrm{ppb}$ ) in comparison to carboxylated SWCNTs (Figure 8) [156]. A lower LOD of $10 \mathrm{ppb}$ and a theoretical LOD of 500 ppt for selective $\mathrm{H}_{2} \mathrm{~S}$ detection have been recently achieved using a FET based on $\mathrm{Au}$ NPs-functionalized ZnO NWs [157]. Au NPs of 1, 3, 5, 7, and $10 \mathrm{~nm}$ were tested, with those of $7 \mathrm{~nm}$ leading to the maximum interaction between the analyte and the sensing film.
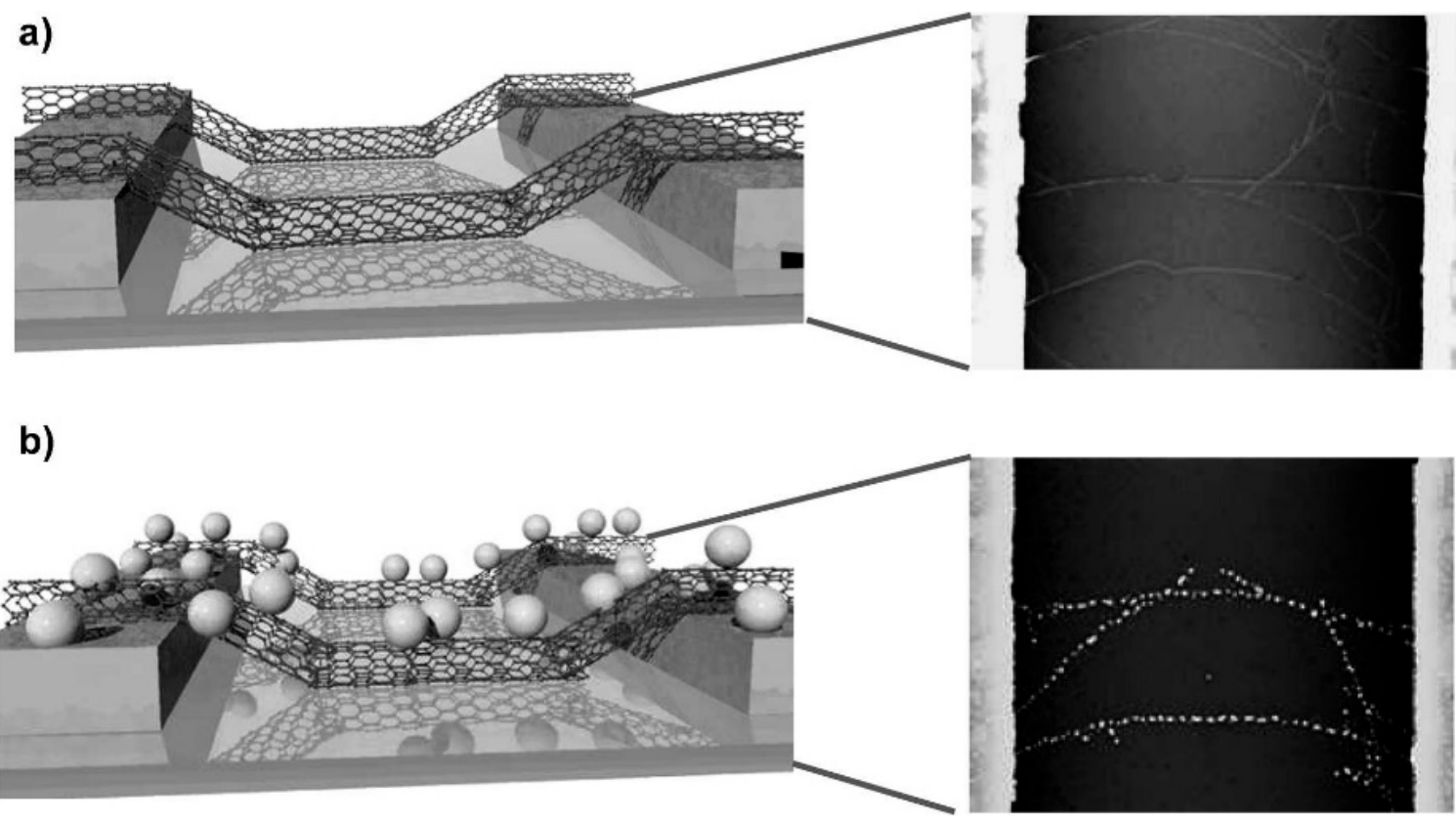

Figure 8. Schematic representation and SEM images of (a) carboxylated SWCNTs aligned between Au electrodes and (b) Au-decorated SWCNTs, as sensing films for gas sensors (FETs), for enhanced $\mathrm{H}_{2} \mathrm{~S}$ sensing. Au NPs play a crucial role in device-performance by modulating the mobility after the gaseous-molecule interactions. Reprinted with permission from Ref. [156]. Copyright (c) 2011 Wiley-VCH Verlag GmbH \& Co. KgaA, Weinheim.

\subsubsection{Cross-Reactive Applications}

NWs-FET sensors comprise the most common type used for cross-reactive VOCs detection, including FETs based on SiNWs and MOS NWs (e.g., $\left.\mathrm{SnO}_{2}, \mathrm{ZnO}, \mathrm{In}_{2} \mathrm{O}_{3}\right)[63,142]$. Using pristine SiNWs, it is possible to discriminate VOCs with high dielectric constants, such as methanol, ethanol, and 2-propanol, with 96\% accuracy and acetone, ethanol, and water with $100 \%$ accuracy by using pattern recognition methods [158]. As far as MOS NWs are concerned, n-type MOS have been mainly studied. $\mathrm{SnO}_{2} \mathrm{NWs}$, e.g., have been used for acetone, ethanol, and methyl ethyl ketone discrimination; the modulation of gate voltage as well as of the operating temperature, permitted the adjustment of sensor response and selectivity [159]. p-Type MOS NWs are also reported to achieve effective VOC detection, such as $\mathrm{CuO} \mathrm{NWs}$ for $\mathrm{NO}_{2}$ and ethanol detection, with ethanol response being reversible by temperature increase due to oxidation towards $\mathrm{CO}_{2}$ and water and electron transfer from water to $\mathrm{CuO}$ NWs [160]. Complementary-MOS based sensors have also been investigated, along with pattern recognition methods, especially for acetone, acetic acid ethanol, propanol, butanol, and hexanol discrimination [161]. In this case, only one sensor can be used, rather than a sensor array, as selectivity can be achieved by alternating the drain-source and gate potential and without any further modification [161]. 
Concerning FET arrays based on different nanomaterials, n-type semiconducting $\operatorname{In}_{2} \mathrm{O}_{3}$, $\mathrm{SnO}_{2}$, and $\mathrm{ZnO}$ NWs combined with a SWCNT-FET have successfully discriminated $\mathrm{H}_{2}$, $\mathrm{NO}_{2}$, and ethanol [162]. The 4-sensor array was tested at both $200{ }^{\circ} \mathrm{C}$ and $\mathrm{RT}$ as well as at different analyte concentrations, while SWCNT-FET incorporation improved ethanol and $\mathrm{H}_{2}$ overlapping [162].

Remarkably, molecular functionalization has been reported, specifically by Haick et al., for particularly promising FET applications. Molecularly functionalized random CNTs networks have been used for nonpolar and polar VOCs detection. As representative LC biomarkers, decane and 1,2,4-trimethylbenzene have been effectively detected by molecularly modified CNTs [163]. Notably, it has been observed that CNT-functionalization determined the semiconducting character of the material, with tricosane-CNTs leading to a p-type and pentadecane/dioctyl phthalate-CNTs to a n-type behavior thus affecting signal responses [163]. More interestingly, molecularly functionalized SiNWs have been used for cross-reactive FET sensors, targeting exhaled volatile organic biomarkers not only in synthetic but also in real-world breath samples; the discrimination of GCa patients from HC [125] as well as the discrimination of patients with asthma/COPD, LC and GCa (Figure 9) with remarkably increased accuracies [43] (Table 2) are noteworthy examples. As is to be expected, chemical functionalization of different nanomaterials allows for the detection of both polar and non-polar VOCs.

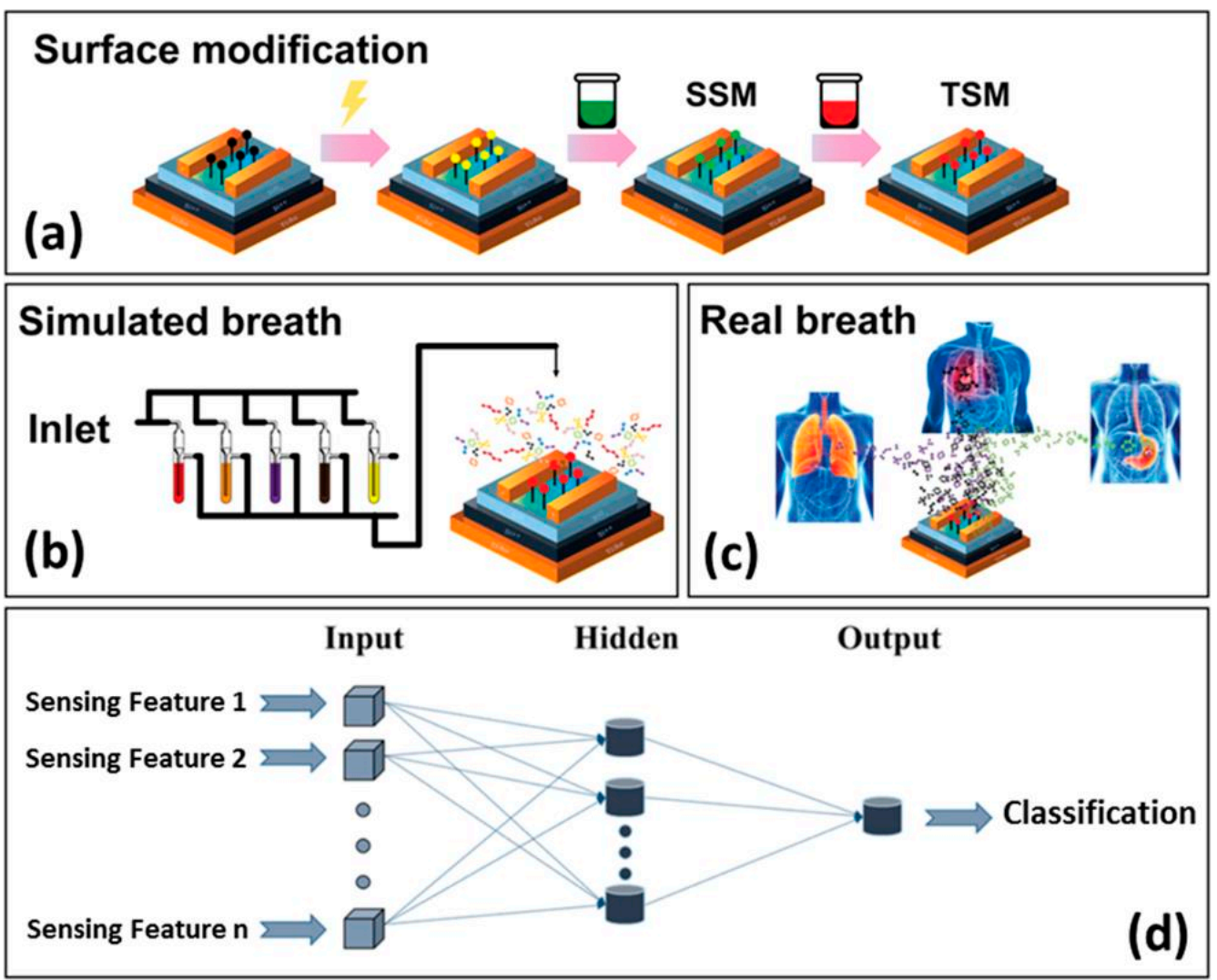

Figure 9. Schematic of (a) surface modification of SiNW FET sensors (SSM, single-step modification; TSM, two-step modification); (b) sensor exposure to synthetic samples of potential organic volatile biomarkers for each disease (asthma/COPD, LC, GCa); (c) sensor exposure to real breath samples of patients with selected diseases, in comparison to $\mathrm{HC}$; (d) ANN analysis representation. Increased accuracies were obtained for the discrimination of GCa vs. LC and LC vs. asthma and COPD patients. Reprinted with permission from Ref. [43]. Copyright @ 2016, American Chemical Society. 


\subsection{Electrochemical Sensors}

Electrochemical sensors are divided into potentiometric (voltage measurement), amperometric (electric current measurement), or conductometric (conductivity or resistivity measurement) [143]. Analyte detection occurs on appropriate electrodes on which a chemical reaction (oxidation or reduction) takes place. Electrochemical sensors typically consist of a sensing (working) electrode and a counter electrode separated by a thin layer of electrolyte [51]. The sensing electrode, on which the reaction occurs, is characterized by high surface-to-volume ratio (for signal enhancement) and is composed of catalytic materials, e.g., platinum, palladium, or carbon-coated metals [143] that are specific for the desired analyte [51]. Analyte-electrode reaction generates a sufficient electrical signal [51] measured with respect to the counter electrode [143]. A schematic representation of electrochemical sensors is presented in Figure 4.

\subsubsection{Selective Applications}

Electrochemical sensors have mainly been used for the selective detection of gas biomarkers. To this end, conventional, polymeric and hybrid materials have been studied. For example, Prussian Blue electro catalyst-modified carbon electrodes on wearable, paper-based sensor have been developed for $\mathrm{H}_{2} \mathrm{O}_{2}$ detection which serves as a lung-disease biomarker [164]. As an example of polymeric sensing films, cylindrical nano-porous semiconducting polymers have permitted $\mathrm{NH}_{3}$ detection in ppb levels via a redox reaction [165]. Hybrid materials, especially the ones containing 2D nanosheets, are also reported in the literature for sensing oxygen-compounds. Nonanal detection for instance has been achieved using $\mathrm{SnO}_{2}$ nanosheets decorated with $\mathrm{SnO}_{2} \mathrm{NPs}$ as well as noble metal catalysts $(\mathrm{Pt}, \mathrm{Au}$, and Pd), aiming at LC early-diagnosis [59]. Solid proton-conducting electrolyte based on sulfonic acid co-functionalized cellulose NFs and GO nanosheets, has been developed for ethanol detection via oxidation with a LOD of 25 ppm [166]. More recently, Au NPsdecorated $\mathrm{MoS}_{2}$ nano-flakes were used for oxygen-based VOCs detection such as the diabetes biomarker acetone, with sensor responsiveness and selectivity being increased due to electron-donation from Au NPs to $\mathrm{MoS}_{2}$ (Figure 10) [69].

(a)

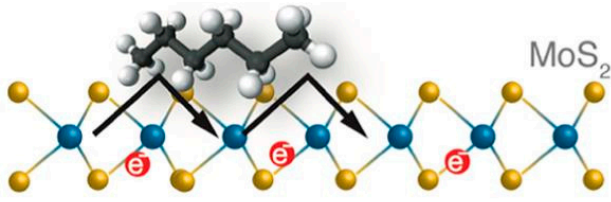

(c)

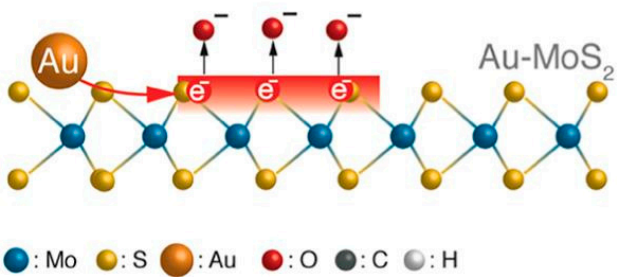

(b)

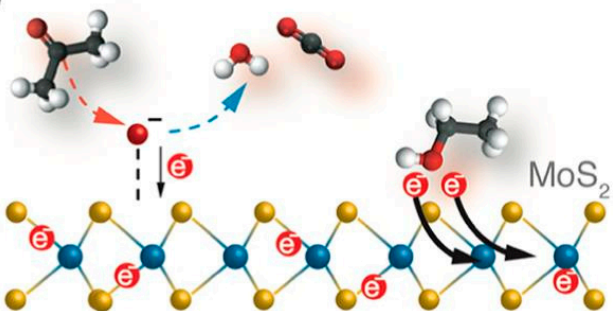

(d)

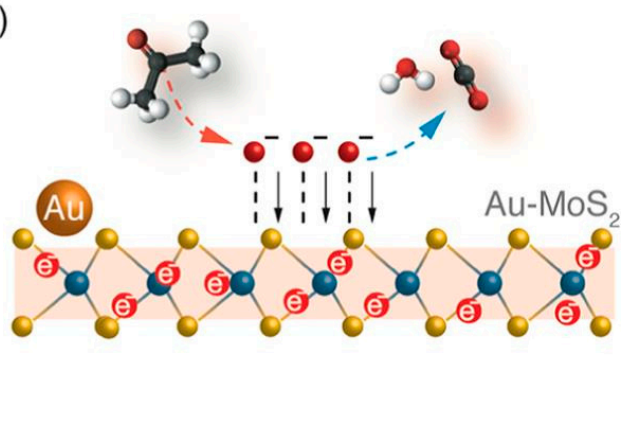

Figure 10. Schematic representation of Au-MoS 2 nano-flakes sensing mechanisms for (a) hydrocarbons using $\mathrm{MoS}_{2}$, leading to small signal alternation, due to dipole scattering through the electron clouds of $-\mathrm{CH}_{2}-$ groups; (b) oxygen-based VOCs using $\mathrm{MoS}_{2}$, which donate electrons to the $\mathrm{MoS}_{2}$ 
(left) and are oxidized by the adsorbed oxygen species, releasing electrons to $\mathrm{MoS}_{2}$ (right); (c) oxygenbased VOCs using $\mathrm{MoS}_{2}$ decorated with Au NPs, which increase electron concentration and, thus, oxygen species adsorption on $\mathrm{MoS}_{2}$; (d) returning more electrons upon interaction with the oxygenbased VOCs. Reprinted with permission from ref. [69]. Copyright (c) 2019, American Chemical Society.

\subsubsection{Cross-Reactive Applications}

Cross-reactive electrochemical sensors have been reported for the in vivo/in vitro diagnosis of diabetes, LC [126], and GCa [137] (Table 2); such devices are based on polymers [126] and nanomaterials (e.g., MNPs, CNTs, SiNWs, graphene derivatives, and their combinations). Remarkably, ultrahigh sensitivity for two GCa biomarkers (Table 2) has been achieved using an Au-Ag NPs-MW CNTs glass carbon electrode, due to the high surface area of both MWCNTs (Au-Ag NPs adsorption enhancement) and Au-Ag NPs (electron-transfer acceleration) [137]. The synergistic catalytic activity of the bimetallic NPs on the other hand enhances the selectivity [137]. Notably, electrochemical sensors are conventionally constrained in detecting electrically inert simple aromatic compounds and hydrocarbons, since the target analyte should be electrochemically active [51]. More recently, the detection of cyclohexane along with formaldehyde, has been reported using a Si NW-rGO sensing film, due to cyclohexane-oxidation catalysis by rGO [106].

\subsection{Piezoelectric Sensors}

In general, piezoelectric materials produce voltage due to the application of mechanical stress and vice versa [51]. Piezoelectric sensors, by definition sensitive to mechanical stress [15], are often used as mass-sensitive sensors [36]. Acoustic wave devices are used in piezoelectric sensors, also called mass, gravimetric, or microbalance sensors. An oscillating circuit is used for the generation of acoustic waves, allowing the piezoelectric crystal to resonate [143]. The most important categories of piezoelectric gas sensors are QCM and SAW [40].

\subsubsection{Quartz Crystal Microbalance (QCM) Sensors}

QCM sensors possess quartz crystal resonators functionalized with different appropriate sensing elements (e.g., metalloporphyrins [131,132], sensitive polymers, MOS, or nanomaterials) $[6,40,167]$. The acoustic wave propagates through the bulk of the crystal perpendicularly or parallel to the surface [51]. When a gas is absorbed on the sensitive surface of a crystal the mass changes thus alternating the resonance frequency [167]. Typically, mass increase results in a decrease in the oscillation frequency of the resonator [143] which comprises the measured physical quantity [167]. The sensing mechanism of QCM sensors is presented in Figure 11a.

\section{Selective Applications}

QCM sensors are used extensively for selective gas detection and are potentially applicable in breath analysis. As an example of MOS based sensors, $\mathrm{ZnO}$ has been used in the form of NWs [168] and vertically-aligned $\mathrm{NRs}$ [169] for $\mathrm{NH}_{3}$ detection, permitting the development of reproducible and stable systems in both cases as well as increased selectivity against liquefied petroleum gas, $\mathrm{N}_{2} \mathrm{O}, \mathrm{CO}, \mathrm{NO}_{2}$, and $\mathrm{CO}_{2}$ in the case of NRs. Notably, VOC detection with MOS-QCM sensors is feasible at RT (despite the use of MOS) since it is directly connected to mass-changes [169]. Concerning polymeric materials, for the selective detection of $\mathrm{NH}_{3}$ [170] and formaldehyde [171], the use of polymeric NFs based on poly(acrylic acid)/poly(vinyl alcohol) (PAA/PVA) [170] and polyethylenimine (PEI)/PVA [171], respectively, has been demonstrated. More recently, a Si NPs-containing methacrylic acid-based MIP-composite was reported to selectively detect hexanal as LC biomarker in the presence of trimethyl amine, $\mathrm{NH}_{3}$, ethanol, acetone, acetic acid, and diethyl ether, due to analyte binding through hydrogen bonding formation [74]. 


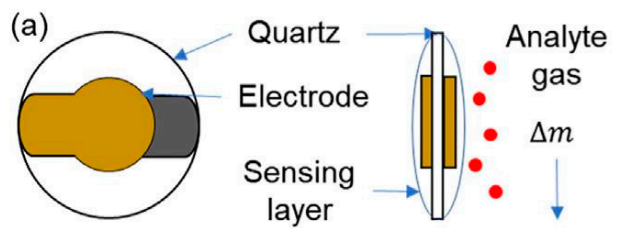

$\Delta f$

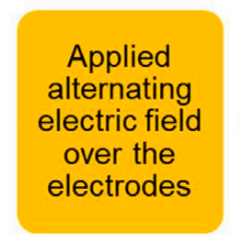

Output IDT
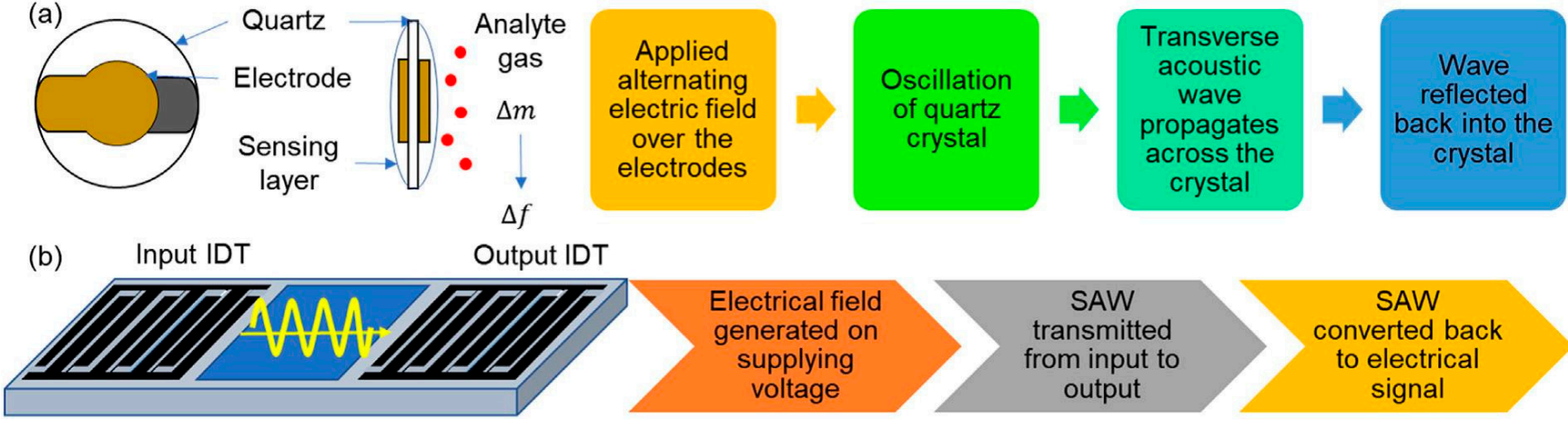

Wave

reflected

crystal
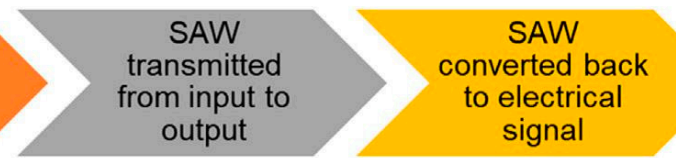

Figure 11. Representation of the sensing mechanism of gas detection using (a) a QCM sensor where the oscillation of the quartz crystal substrate and propagation of the transverse acoustic wave through the quartz substrate are caused by the alternating electric field applied over the electrodes; sensing layer-gas interactions change the mass on the substrate and hence wave amplitude and velocity, leading to a resonance frequency shift $(\Delta \mathrm{m} \rightarrow \Delta \mathrm{f})$; (b) SAW sensors where a surface wave confined within one acoustic wavelength of the surface of the piezoelectric material is induced by an input RF-voltage applied across the interdigitated transmitter (IDT); mechanical energy is transformed back into radio frequency as an output when the SAW reaches the receiving IDTs. Analyte adsorption on the piezoelectric coating induces mass variations and ultimately a shift in frequency. Reprinted with permission from Ref. [172]. Copyright (c) 2021 Elsevier B.V. All rights reserved.

\section{Cross-Reactive Applications}

In the case of cross-reactive applications, hybrid and especially polymeric/macrocyclic sensing materials are investigated. $\mathrm{TiO}_{2} \mathrm{NPs}$-decorated MWCNTs and cobalt (II) phthalocyanine-silica, for instance, have been used as sensing elements in a 3 sensor array, along with a bare QCM sensor; this was used for simultaneous acetone and NO detection, which serve as diabetes and asthma biomarkers, respectively [139]. No response was observed by the bare sensor, while analyte adsorption to the modified sensors is attributed to analyte-sensing film coordination interactions [139]. Concerning polymeric materials, eight different polythiophene derivatives have achieved acetic acid, toluene, acetone, $p$-xylene, ethanol, 1-octanol, acetonitrile, and water discrimination based on VOC-polarity, with a LOD at ppm levels; prediction of toluene concentration in mixtures with ethanol was also permitted [173]. Macrocyclic calixarene derivatives, on the other hand, have achieved the detection of 16 VOCs belonging to ketones, alcohols, aromatic, and chlorinated compounds, at ppm levels, due to "host-guest" interactions. Sensor sensitivity and selectivity were dependent on the number and functional groups of calixarene derivatives [174]. In a more recent application, 13 different MIPs of different compositions and cavity structures were studied for the detection of hexanal, nonanal, and benzaldehyde, which are common cancer biomarkers. A combination of five sensors led to the most effective VOC discrimination while sensor-array performance was found to be dependent on both molecular imprinting and matrix effect [73].

Metalloporphyrin-based cross-reactive QCM sensors seem as the most promising QCM sensors (Table 2) while they have been also applied in real breath samples [130-134]. Such eNoses have been examined for asthma [131], COPD [130], halitosis [135], LC [132,133,175], and tuberculosis [134] diagnosis with particularly promising diagnostic performance. Similarly, an anthocyanin-based QCM sensor called BIONOTE e-Nose has been developed by Santonico et al. [176] and was recently studied for use in asthma diagnosis of children, with the aim to assess the within and between-day repeatability of obtained measurements; values similar to those of conventional methods were achieved (Table 2) [136].

\subsubsection{Surface Acoustic Wave (SAW) Sensors}

In $S A W$ gas sensors, the acoustic wave propagates only parallel to the surface of the piezoelectric crystal, penetrating about one acoustic wavelength in depth into the 
crystal. Motion at the surface is both parallel and perpendicular to it [51]. Crystal surface is modified with a chemically selective layer $[40,143]$. Exposure to the analyte affects the propagation waves [143], as the mass (acoustic field of the SAW) and/or the electrical conductivity (electric field of the SAW, associated with the acoustic field) of the chemical interface change [15]. As a result, a change is induced in the propagation frequency of the SAW, which can then be measured $[6,40]$. The sensing mechanism is presented in Figure 11b.

\section{Selective Applications}

Similar to QCM sensors, MOS including $\mathrm{ZnO}, \mathrm{SiO}_{2}, \mathrm{TiO}_{2}, \mathrm{Co}_{3} \mathrm{O}_{4}, \mathrm{WO}_{3}$, and other combinations [7,172], have been used extensively for SAW devices and most commonly for selective VOCs detection. For example, ethanol detection has been achieved at $300{ }^{\circ} \mathrm{C}$ using a YX $\mathrm{LiTaO}_{3}$-based SAW sensor modified with a $\mathrm{ZnO}$ intermediate layer of $1.2 \mu \mathrm{m}$ and a $\mathrm{WO}_{3}$ sensing layer of $150 \mathrm{~nm}$ [177]; porous $\mathrm{ZnO}-\mathrm{SiO}_{2}$ bilayer nano-films have been used for $\mathrm{NH}_{3}$ detection, with $\mathrm{SAW}$ sensor sensitivity being better than the one for single layers and was found to be dependent on bi-film conductivity [178]. In the latter system, it was observed that increased $\mathrm{ZnO}$ film thickness led to larger absolute sensor response but also to larger response and recovery times; the study concluded that $60 \mathrm{~nm}$ of $\mathrm{ZnO}$ led to the best sensing performance for $30 \mathrm{ppm} \mathrm{NH}_{3}$ [178]. The detection of $\mathrm{NH}_{3}$ concentration levels that were lower than $1 \mathrm{ppm}$ has been also attempted by the same group in the presence of $\mathrm{H}_{2}, \mathrm{CO}, \mathrm{CH}_{4}, \mathrm{H}_{2} \mathrm{~S}$, and ethanol using $\mathrm{SiO}_{2}-\mathrm{TiO}_{2}$ films of $200 \mathrm{~nm}$ that exhibited remarkable system selectivity, stability, and reproducibility [179].

\section{Cross-Reactive Applications}

Non-functionalized $[180,181]$ or polymer-functionalized [129] SAW sensors have been combined with GC columns as detectors for the development of point-of-care diagnostic systems. BC [180], tuberculosis [181], and LC [129] patients for instance, have been effectively diagnosed with an accuracy of $79 \%, 84 \%$, and $80 \%$, respectively. SAW sensors have been also used for the detection of both polar and non-polar VOCs, after their modification with appropriate sensing films [6].

Cross-reactive SAW sensor arrays with polymeric coatings of different composition [182] or thickness [183] have been reported; SAW sensors coated with eight different polymeric films have achieved effective discrimination of chloroform, octane and xylene vapors as shown by radar plots, maintaining their performance for a period of 3 years [182]. Interestingly, 3 polyisobutylene films of varying thickness, namely 10, 50, and $100 \mathrm{~nm}$, allowed for chloroform, chlorobenzene, o-dichlorobenzene, heptane, toluene, hexane, and octane discrimination by analyzing the transient responses characterized by analyte-specific kinetics variability (different stages of equilibrium attainment, for different coating thickness) [183]. Such polymer-based SAW sensors hold a great promise for potential breath analysis applications. CNTs, SWCNTs, and MWCNTs dispersed in ethanol or toluene for example, have been separately tested for ethanol, toluene, and ethyl acetate sensing at RT with a LOD of 1 ppm [184]. The main advantage of CNTs-modified sensors is the enhanced SAW sensor-sensitivity, due to the ability to sense variations not only in mass but also in conductivity [172].

Among hybrid materials, MWCNTs combined with other materials have achieved selectivity enhancement. Polyepichlorohydrin and polyurethane were combined with different MWCNTs percentages so as to develop a 4 sensor-array, for toluene and octane detection and differentiation using polar plots [185]. Notably, no response was observed for gases such as $\mathrm{H}_{2}, \mathrm{NH}_{3}, \mathrm{NO}_{2}$, and $\mathrm{CO}$, while toluene adsorption was far more enhanced than that of octane [185]. In a different publication, MWCNTS combined with nano-sized $\mathrm{CeO}_{2}$ (100:1) have formed a semiconducting composite that achieved acetone and ethanol detection, with higher sensitivity for the former [186]. As far as MNPs-based hybrid materials are concerned, thiol containing calix4arene-modified AuNRs and Ag nano-cubes (NCs) have been recently used in a 5-sensors array, for the detection of polar and non-polar 


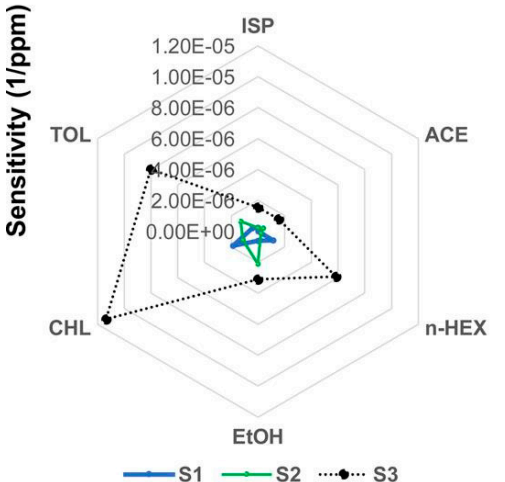

(a)

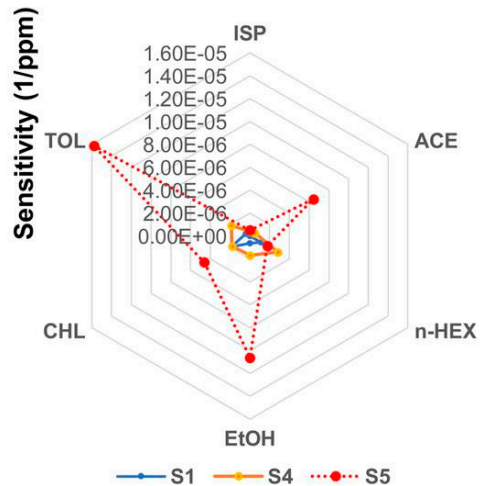

(b)
VOCs in low ppm levels including the LC biomarker toluene. Chloroform and toluene were detected with greater sensitivity by the modified AuNRs and AgNCs respectively when compared to unmodified NPs (Table 2) [49]. Calix4arene modification enhanced sensitivity largely due to the increase of the surface interaction area as well as the arrangement of the macrocyclic ligands (Figure 12) [49]. The use of pristine or Au NPs-functionalized zeolitic-imidazole-framework nanocrystals (ZIF-8 and ZIF-67) in a 4-sensor array for the discrimination of diabetes biomarkers (acetone, ethanol, and $\mathrm{NH}_{3}$ ) via PCA is another example of promising hybrid materials [138] (Table 2). Molecularly functionalized MOSNPs are also reported in the literature. Using a layer of amino-terminated iron oxide NPs for instance, the VOCs butanol, isopropanol, toluene, and xylene were detected with a low LOD of $1,12,3$, and 0.5 ppm, respectively, thus developing an effective sensing device of lower cost in comparison to MNPs-based sensing systems [187]. Iron oxide NPs have been also used in combination with polymers. $\mathrm{Fe}_{3} \mathrm{O}_{4}$ NPs of varying diameter $(7$, 13 , or $50 \mathrm{~nm}$ for $0.4 \mathrm{mg}$ NPs $/ \mathrm{mL}$ polymer solution) and concentration $(0.2,0.4$, or $0.8 \mathrm{mg}$ NPs/mL polymer solution for NPs $50 \mathrm{~nm}$ ), have been embedded in PEI [52]; by employing a PEI functionalized sensor, a 6-sensor array was developed for the effective detection of methanol, ethanol, and toluene while sensitivity increased for smaller NP-diameters and greater concentrations. Remarkably, NPs of $7 \mathrm{~nm}$ permitted ethanol detection with a LOD five times better (65 ppm) than solely PEI-based sensors [52].

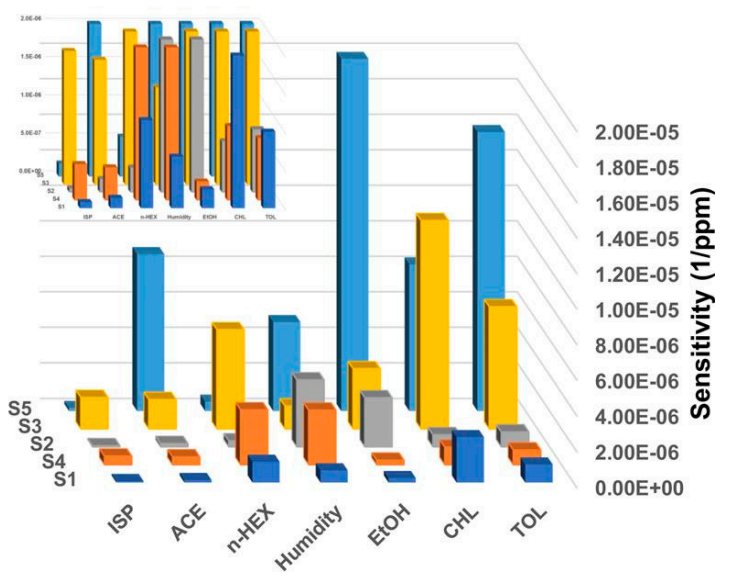

(c)

Figure 12. Sensitivity values (1/ppm) of (a) sensors S1-S3, (b) sensor S1-S4-S5 in radar plots, and (c) sensors S1-S5 in bar chart representation, for six different analytes under $0 \% \mathrm{RH}$ provided with responses to $100 \%$ humidity. Inserted graph: magnified view of data below $2 \times 10^{9} 1 / \mathrm{ppm}$. (S1: SH-Calix[4]arene, S2: AuNRs, S3: Calix[4]arene modified AuNRs, S4: AgNCs, and S5: Calix[4]arene modified AgNCs, ISP: isoprene, ACE: acetone, n-HEX: n-hexane, EtOH: ethanol, CHL: chloroform, TOL: toluene). Calix[4]arene modification (S3, S5) increased the sensitivity, under $0 \%$ of humidity, especially for TOL and CHL, while thiol terminated calix[4]arene (S1) exhibited increased response towards CHL. S5 exhibited the highest responsiveness towards TOL among other VOCs and the highest among all sensors ( $\pi-\pi$ interactions leading to host-guest complexes). Reprinted with permission from Ref. [49]. Copyright (C) 2021 Elsevier B.V. All rights reserved.

In comparison to QCM sensors, SAW based sensors are generally characterized by higher sensitivity, while the potential for surface modification is expanded. On the other hand, it is worth noting that in both cases apart from the sensing film composition that determines sensor selectivity, the high surface area of the nanostructures comprises the fundamental factor that enhances sensitivity due to the creation of more adsorption sites (defects) $[52,169,171,187]$. In general, piezoelectric gas sensors, investigated primarily in synthetic samples, are characterized by increased sensitivity, small response time and low-powered operation. However, the low signal-to-noise ratio and the requirement for 
complex electronic circuits, may render this sensor type less enticing for the development of efficient e-Noses [40].

\subsection{Optical Sensors}

Optical gas sensors detect analytes by measurable changes of absorption, luminescence, scattering, reflectivity, refractive index or optical path length [40], due to the interaction of the radiation with the desired gas or a selective layer [143]. In the first two cases, light intensity or wavelength are measured [40].

\subsubsection{Optical Fiber-Based Sensors}

Optical fibers possessing a chemical reagent (e.g., chemical dye) or a sorbent phase (e.g., polymeric film) as a reactive layer, are commonly used as transduction elements in optical gas sensors [6,143] specifically for VOC detection [6]. Upon vapor exposure optical or structural changes of the reactive layer alter the effective index and hence the light transmission properties of the fiber [6].

\section{Selective Applications}

Nanomaterial-based hybrid materials have been used for optical fiber modification as sensing films for selective VOC detection. The use of graphene, commonly combined with MNPs [188,189], is a representative example. Pt NPs-GO [188] and Ag NPs-GO [189] functionalized optical fibers have been developed for efficient and selective $\mathrm{NH}_{3}$ detection $\left(\mathrm{NH}_{3}\right.$ is a common biomarker for renal and liver diseases as well as Helicobacter pylori infection) [7]. Remarkably, Pt NP-functionalization increased the sensitivity in comparison to pristine GO [188], while the concentration of Ag NPs was inversely correlated with sensitivity [189]; this reveals the benefits as well as the vulnerability of the synergistic effects of hybrid materials. An additional hybrid material, namely a thin film of poly (allylamine hydrochloride) and Si NPs infused with tetrakis (4-sulfophenyl) porphine, has been used as an optical-fiber coating for the selective detection of methanol in the presence of water and other alcoholic vapors [190].

Cross-Reactive Applications

Standalone polymers or polymers combined with nanomaterials have also been used for cross-reactive applications. PMMA-based fibers functionalized with nano-crystalline bismuth oxide-clad have effectively detected $\mathrm{NH}_{3}$, ethanol, methanol, and acetone, exhibiting increased selectivity towards methanol [191]. Detection of hydrocarbons and aromatic compounds in real breath samples of $\mathrm{HC}$, as potential biomarkers of various diseases, has been also achieved using a polymethyl (3,3,3-trifluoropropyl) siloxane-coated optical fiber; the device features a low LOD posing as an attractive alternative for disease diagnosis (Table 2) [128]. Despite their extensive investigation in the field of breath analysis for VOCs detection, such devices are scarcely studied for real sample experiments [6].

\subsubsection{Colorimetric}

Colorimetric sensors, usually classified as a sub-group of optical sensors [51], are based on environmentally dependent color changes [15]. Chemo-responsive indicators are able to chemically react and change color in a distinctive way upon exposure to different gas analytes [6], thus permitting analyte identification. In this case the response upon analyte exposure is based not on the physical properties but the chemical reactivity of the indicators [15].

\section{Selective and Cross-Reactive Applications}

Selective applications of colorimetric sensors have scarcely been studied. Lead acetate $\left(\mathrm{PbAc}_{2}\right)$ NPs anchored to polyacrylonitrile nanofibers (NFs) comprise an example of a selective sensing element that was investigated for $\mathrm{H}_{2} \mathrm{~S}$ colorimetric detection (a halitosis biomarker); a LOD of $400 \mathrm{ppb}$ which is far lower than the $5 \mathrm{ppm}$ of $\mathrm{PbAc}_{2}$ paper tests, 
was achieved [192]. Concerning cross-reactive sensing systems, there are five possible categories to which an indicator may belong to: $\mathrm{pH}$ responsive, (Brønsted acidity/basicity); metal-ion-containing dyes (Lewis basicity-electron pair donation); redox-responsive metal salts; nucleophilic indicators (responsive to electrophilic analytes) and dyes with large permanent dipoles (e.g., solvatochromic dyes) [6]. Such optical sensors have been extensively investigated in the field of breath analysis, mainly for LC diagnosis, using both synthetic [140,193] and real-world breath samples [127,194]. Hou et al. have achieved to discriminate at first 4 (Table 2) [140] and then 20 [193] LC-related VOCs in two separate studies, with accuracies of $100 \%$ and $90 \%$, respectively. Mazzone et al. achieved to differentiate LC patients and HC, with moderate accuracies [127,194], as well as LC patients of different histologies with a higher accuracy percentage (Table 2) [127]. The main challenges for such sensors include low sensitivity, high LOD and low response times. Furthermore, their irreversible operation renders them disposable (single-use tests), which is something that should also be considered [7,167]. Nanomaterials may be also used for enhanced sensing properties. For the detection of VOCs that have been identified as LC biomarkers the use of AuNRs-modified metalloporphyrins has proven to protect the device from photo-degradation, to provide good repeatability, increased long-term sensor stability, and increased shelf-life (Figure 13) [141].
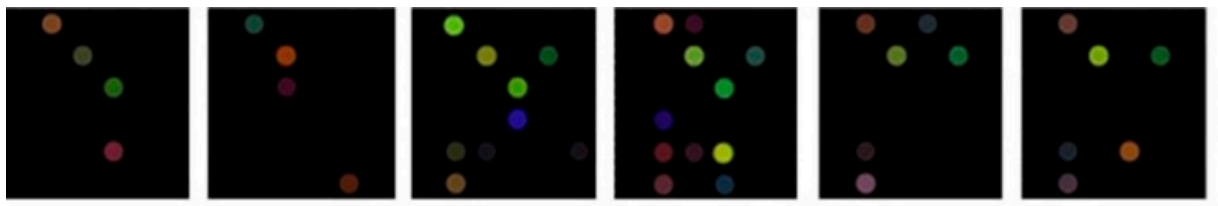

$0.75 \mathrm{~mL}$
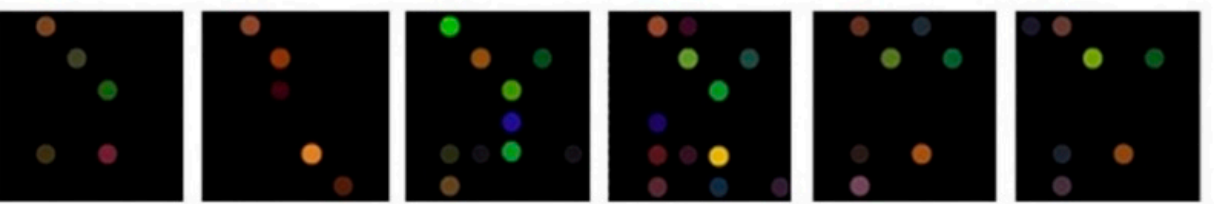

$1.5 \mathrm{~mL}$

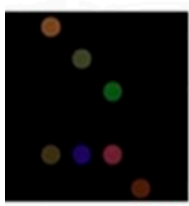

Decane

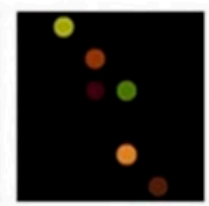

Undecane

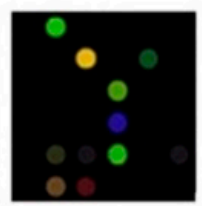

Heptanal

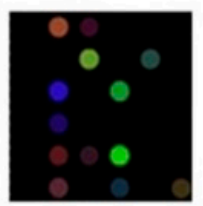

Hexanal

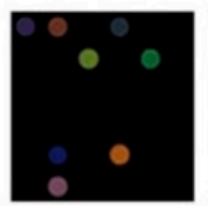

Benzene

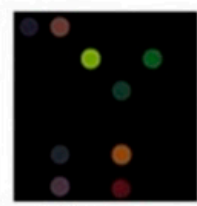

$1,2,4-$

Figure 13. Detailed color difference maps of six VOCs at three volumes $(0.75,1.5$, and $3 \mathrm{~mL}$ saturated vapor, respectively) saturated analyte vapor at $20^{\circ} \mathrm{C}$, acquired by metalloporphyrin-AuNRs and dyesbased optical chemical sensor. Metalloporphyrin-AuNRs exhibited increased responsiveness and high sensitivity and selectivity due to their high affinity. Reprinted with permission from Ref. [141] Copyright (C) 2014 Elsevier B.V. All rights reserved.

\subsubsection{Localized Surface Plasmon Resonance (LSPR)-Based Sensors}

Non-conventional chromophores, such as MNPs, have also been investigated in optical sensors, due to their interesting optical properties. LSPR-based gas sensors $[36,40]$ are based on the dependence of the LSPR properties of the MNPs from the refractive index of the dielectric environment surrounding the NP [36] (i.e., coating, surrounding medium, supportive substrate [51]). Refractive index alternation of the medium changes the wavelength of the incident light [36]. The main advantage of MNPs is the fact that the extinction coefficients are several orders of magnitude higher than those of conventional dyes, in the visible spectrum, allowing higher sensitivity and lower LOD for the desired analyte [51].

Optical detection of VOCs using various compositions and shapes of MNPs has also been reported. Au NPs, Ag NPs, and Au NSs have been used for chlorobenzene, $m$-xylene, 
pentanol, toluene, and octane cross-reactive detection [195]. Hybrid materials containing MNPs have been used as well. Selective polymer-coated Au nano-islands, for instance, have effectively detected a-pinene [196].

\subsubsection{Surface Enhanced Raman Spectroscopy (SERS)-Based Sensors}

Another, more widespread optical-sensor application of the LSPR phenomenon in MNPs, is SERS [36], i.e., the enhancement of Raman signals due to the LSPR phenomenon. SERS is a vibrational spectroscopic technique [197] that permits single-molecule detection, and has been investigated among others for VOCs detection [198].

Functionalization of different MNPs with appropriate molecules has been reported for selective VOC-detection. Aldehydes detection as LC biomarkers by SERS-based sensors seems promising [198,199]. Dendritic Ag nanocrystals coated with the aldehyde-selective probe molecule $p$-aminothiophenol have achieved low LOD (ppb range) in the presence of confounding LC biomarkers (hydrocarbons, alcohols, ketones, esters, nitrogen, and aromatic compounds) [199]. NO, on the other hand, serves as a biomarker for asthma [36], hypertension, arteriosclerosis, diabetes, and rheumatoid arthritis [197]. NO detection has been achieved by $o$-Phenylenediamine-modified Au NPs which have permitted selective chemical reaction between functionality moieties and NO, leading to nano-probe SERS variations; such sensors have achieved a LOD of $1.7 \times 10^{-7} \mathrm{M}$, in the presence of $\mathrm{H}_{2} \mathrm{~S}$ and CO [197].

As it can be observed, SERS-based sensors have been developed for the selective detection of a specific VOC or classes of VOCs, rather than cross-reactive detection. Notably, optical sensors are not preferred for e-Nose development, mainly due to their size and complex signal conditioning [40].

On Table 2 representative examples for different types of sensor-arrays, using conventional materials or nanomaterials, are presented. Sensitivity, selectivity, and discriminant accuracy of the sensor arrays highlight their promising application as diagnostic tools. It can be observed that the incorporation of nanomaterials in the sensing element ameliorates the sensing performance (i.e., sensitivity, LOD), while appropriate modification permits the desired cross-selectivity. Sufficient LOD, similar to the usual concentration of VOCs in exhaled breath, is achieved by all sensor types. As far as MOS-based sensors are concerned, it is apparent that their main drawback towards all the other categories is their increased operating temperature. Chemiresistors are probably the most investigated gas sensor type for cross-reactive systems, incorporating different (nano)materials, being particularly attractive for the diagnosis of a wide range of diseases. Remarkably, most of the branded e-Noses that have been developed and tested for breath analysis applications, presented on Table 3, appertain to chemiresistors (AeoNose, Cyranose 320, SpiroNose, DiagNose, and Common Invent e-Nose). Another noteworthy information presented on Table 2 is the variety of data analysis and machine learning techniques used among the different studies, in the absence of which the discrimination of subjects would be infeasible. 
Table 3. Branded e-Noses and type of technology used in each case.

\begin{tabular}{cccc}
\hline Electronic Nose & Technology Used & Ref. & \\
\hline Cyranose $\mathbf{3 2 0}$ & 32 carbon black-polymer composite chemiresistors & MOS sensors & {$[117,118]$} \\
\hline Common Invent e-Nose & 5 sensor arrays, each composed of 4 MOS sensors & Micro hotplate MOS & {$[17]$} \\
\hline SpiroNose & 12 doped MOS & {$[92,117]$} \\
\hline AeoNose & 8 QCM sensors using anthocyanin-coated gold electrodes & {$[93,118]$} \\
\hline DiagNose & QCM covered with metalloporphyrins & {$[117,136]$} \\
\hline BIONOTE e-Nose & Field asymmetric ion mobility spectrometry & {$[117]$} \\
\hline Tor Vergata e-Nose & [117] & \\
\hline Owlstone Lonestar e-Nose & e-Nose: Electronic Nose, MOS: Metal Oxide Semiconductor, QCM: Quartz Crystal Microbalance.
\end{tabular}

\section{Differential Diagnosis and Disease Phenotyping and Staging in Breath Analysis}

As can be observed on Table 2, exhaled VOCs have been used not only for disease diagnosis in comparison to healthy subjects, but also for the discrimination between different diseases (e.g., GCa, LC, and asthma/COPD [43]) or even for the discrimination between the different stages of a particular disease (e.g., CKD stages [113]). One of the main prerequisites for the development of clinically applicable diagnostic tests is the effective discrimination between different diseases with similar symptoms and biochemical pathways [200]. The uncertainty in the differentiation of patients with distinct diseases comprises one of the main drawbacks for studies distinguishing a specific disease from HC [201]. Thus, the use of breath analysis for differential diagnosis as well as disease staging or phenotyping, using either analytical techniques or sensing devices (Table 4), attracted significant research interest over the last years. As expected, the contribution of nanomaterials is of great importance with many recent publications of nanomaterial-based sensors focusing on disease differentiation, staging or phenotyping, rather than the simple discrimination between patients and HC (Table 4).

\subsection{The Case for Lung Diseases}

Chronic and acute lung diseases such as asthma, COPD, idiopathic pulmonary fibrosis (IPF), LC, mesothelioma, and sarcoidosis have been connected with similar metabolic alternations [202]. Especially asthma and COPD are also characterized by similar symptoms [37] with COPD being commonly underdiagnosed or diagnosed at late stages [203]. Concerning LC, no symptoms are expressed in early stages [204] while disease manifestation is limited to non-specific symptoms [204] including cough, short breath, chest pain, and weight loss [37]. Disease phenotyping, on the other hand, is mandatory in some cases. Asthma subtypes such as eosinophilic, neutrophilic, mixed granulocytic, and paucigranulocytic asthma [205] are characterized by similar symptoms while different treatment is required [206]. Similarly, immunosuppressive, antifibrotic, or a combination of medications may be needed for fibrotic interstitial lung diseases (ILDs), depending on the respective phenotype (inflammatory, more fibrotic, or combination) [207]. Thus, reliable phenotyping is needed for appropriate medication to be administered [33,206,207]. LC is subdivided into different categories with different clinical characteristics as well. Small cell LC (SCLC), with 20-25\% percentage of occurrence [208], is characterized by increased metabolic and proliferation rates compared to other cancer cells [209], while non-small cell LC (NSCLC) accounts for $70-75 \%$ of LC cases and is subdivided into the smoking-related $[37,210]$ squamous cell carcinoma (SCC) [208] and the non-squamous cell carcinomas [37] including adenocarcinomas (ADC) (minor smoking correlation) and large-cell carcinoma (LCC) [208]. Consequently, the accurate discrimination of different lung diseases and subtypes of a lung disease, especially using breath analysis of exhaled VOCs, is of particular importance. 
The use of GC-MS has rendered lung disease differentiation, phenotyping and staging feasible in many cases. To be more specific, LC discrimination from patients with other lung diseases has been investigated in several studies. In an attempt to discriminate between NSCLC, COPD, and HC patients, by taking smoking habits into account, 4 VOCs were identified (in varying concentrations) for NSCLC and COPD [211]. In another study, Wang et al. attempted to discriminate LC from COPD, asthma, pneumonia, pulmonary embolism and benign lung tumor patients; however, the selected 10 VOCs could not discriminate accurately between the two groups, implying their potential confounding role during LC-biomarkers determination [18]. Koureas et al. have also attempted to discriminate LC from other respiratory diseases, using 19 distinctive VOCs, based on the underlying disease mechanisms (targeted method); only the discrimination of LC patients from HC, using ethylbenzene, toluene, styrene, 2- and 1-propanol was achieved [200]. However, in a more recent study of the same group, the discrimination of LC patients from patients suffering from sarcoidosis, hypersensitivity pneumonitis, interstitial lung diseases or pulmonary infections was achieved with an increased accuracy of $75.3 \%$; the 29 VOCs were selected following a hypothesis-generating non-targeted strategy [212]. In a different study, LC was accurately distinguished from pulmonary non-malignant diseases (PNMD; COPD, pulmonary tuberculosis, asthma) using 10 VOCs while 5 were selected as characteristic of LC in contrast to both PNMD and HC [213].

The differentiation of LC patients from patients with benign pulmonary nodules (BPN) has also been extensively reported. Apart from a study by Wang et al. [18] which included patients with benign lung tumors, $\mathrm{Fu}$ et al. have investigated the respective discriminant ability of carbonyl VOCs [214-216]. Four carbonyl VOCs, captured by a silicon micro-reactor, were found to present increased concentration in LC patients when compared to BPN patients and HC [214]. In a subsequent study, the same group achieved the differentiation of both early and III, IV stage LC patients from BPN patients, with high sensitivity (83\%) and particularly increased specificity $(74 \%)$ in comparison to positron emission tomography (90\% and 39\%, respectively) [215], while 6 carbonyl VOCs have permitted a classification accuracy of $89 \%$ of LC vs. BPN patients [216]. More recently, Chen et al. identified 19 VOCs able to distinguish not only LC and BPN patients (this with an accuracy of $80.9 \%$ ) but also early-stage LC patients from BPN (with an accuracy of $75.6 \%$ ), being remarkably promising for early LC diagnosis [204].

LC histology and staging characterization using analytical methods is another important target of this research field. It was reported that 1-butanol, 3-hydroxy-2-butanone [9], as well as 4-hydroxyhexenal [214], can differentiate SCC from ADC patients with the former being decreased for SCC in contrast to the other 2 VOCs. Similarly, SCLC and NSCLC can be potentially distinguished from 4-hydroxynonenal and $\mathrm{C}_{5} \mathrm{H}_{10} \mathrm{O}$ [214]. Hexanal has also been found in higher concentrations for SCLC patients compared to NSCLC, potentially due to increased metabolic rates [209]; Chen et al. have achieved NSCLC and SCLC differentiation, with an accuracy of $93.9 \%$, using a pattern of 20 VOCs [204]. Concerning LC staging (I, II, III, or IV), a pattern of 19 VOCs was used to distinguish between early (I, II) and advanced LC stages (III, IV) with 82.7\% accuracy [204] while Fu et al. demonstrated that exhaled 2-butanone concentration is significantly different between stages I and II-IV [214].

Apart from LC, other lung diseases are also studied for accurate diagnosis. A series of studies have focused on asthma phenotyping using GC-MS. Brinkman et al. identified 3 VOCs significantly correlated with sputum eosinophils [90], while Ibrahim et al. identified VOC-patterns differentiating eosinophilic from non-eosinophilic (6 VOCs) and neutrophilic from non-neutrophilic (7 VOCs) [217]. Recently, Schleich et al. identified 4 VOCs discriminating eosinophilic from neutrophilic, eosinophilic from paucigranulocytic and neutrophilic from paucigranulocytic asthma, with accuracy similar to blood eosinophils and FeNO tests [205]. In a more recent study, the same group used two-dimensional GChigh resolution-time-of-flight-MS, selected ion flow tube mass spectrometry (SIFT-MS), 10 VOCs, and 9 ion channels so as to achieve asthma phenotyping with an accuracy of 75\% [206]. COPD phenotyping and staging has also been attempted using analytical tech- 
niques. Fens et al. identified 8 eosinophils- and 17 neutrophils-related VOCs, with only one VOC overlapping between the two subgroups. More VOCs were related with cell counts for Global Initiative for Obstructive Lung Disease (GOLD) stage II, in comparison to GOLD stage I [120]. In another study, 11 COPD patients with $>1 \%$ and 6 with $>2 \%$ eosinophil count were discriminated from non-eosinophilics $(<1 \%$ and $<2 \%$ eosinophil count, respectively) with accuracies of $79 \%$ and $92 \%$ [218]. Exacerbation prediction of both asthmatic children [219-221] and adults [90,217] as well as COPD patients [222] also comprises a subject of study.

Following the promising applications of analytical methods that highlight the potential capabilities of breath analysis in phenotyping, staging and differential diagnosis of lung diseases, sensing devices have also been used in respective applications with remarkable results. Research interest has focused on LC discrimination from other lung diseases such as COPD and asthma. Cyranose 320 has achieved separation of NSCLC from COPD (GOLD stage I-III) with an accuracy of $85 \%$, in an article by Dragonieri et al. [223]. Tirzite et al. have used this e-Nose to effectively discriminate, not only LC patients from COPD, asthma, pneumonia, pulmonary embolism, benign lung tumor patients, and $\mathrm{HC}$, with $87.3 \%$ accuracy, but also between LC patients, COPD patients, LC patients suffering also from COPD and $\mathrm{HC}$ with 77.4\% accuracy, and totally correct classification of the 79 LC/COPD patients [224]. In a more recent study, the same group discriminated LC from patients with non-malignant lung diseases as well as bronchiectasis, tuberculosis, and HC by taking into account smoking habits. An overall sensitivity and specificity of $95.8 \%$ and $92.3 \%$ for smokers and $96.2 \%$ and $90.6 \%$ for non-smokers, respectively, was observed using Cyranose 320 [225]. More recently, the same e-Nose was used by Rodriguez et al. for the discrimination of COPD from $\mathrm{LC}$ and $\mathrm{BC}$, achieving an overall correct classification of $91.35 \%$ while LC correct classification in relation to COPD was equal to $96.47 \%$ [201]. Interestingly, the contribution of the 32 sensors in the discrimination was also assessed [201]. Tor Vergata e-Nose has been used effectively for discriminating LC patients from COPD, Interstitial lung disease, Pleurisy and Bronchitis patients, with a sensitivity of $89.3 \%$ for LC patients [175]. In a particularly promising study, SpiroNose discriminated LC, asthma, COPD, and HC, with the respective accuracy values presented in Figure 14a (68-88\%) [91]. The applicability of breath sampling and analysis was tested as the collection of asthma breath samples at two different sites led to similar results (Figure 14b) [91]. In the field of non-commercial and self-developed sensors, Tan et al. have attempted to develop a cross-reactive alkane-based chemiresistor combining carbon powder and tetracosane, achieving not only high affinity for alkanes and low sensitivity for polar VOCs (water, ethanol, ethanal) but also effective differentiation of $12 \mathrm{LC}$ patient from $13 \mathrm{HC}$ and $12 \mathrm{COPD}$ patients [108]. In a more recent study, researchers attempted to differentiate LC, COPD, and asthma patients from $\mathrm{HC}$, using an array of 8 sensors of 4 different types (MOS, electrochemical, hot wire, and catalytic type). The array achieved accuracy between 76.9-84.75\%, using different machine learning methods [226]. Accuracy values were greater for LC and COPD prediction; however, the maximum accuracy value of $84.75 \%$ was attained using kernel principal component analysis-extreme gradient boosting (KPCA-XGBoost), which indicates excellent discriminatory capability for LC and COPD patients [226]. Similarly, using an array of 11 sensors of 4 different types (namely MOS, electrochemical, hot wire, and catalytic type), Liu et al. differentiated non-smoking LC and COPD patients, with the best discriminatory accuracy $(96 \%)$ being achieved using the same machine learning technique [227]. The discrimination of LC from asthma and COPD patients was also achieved by Haick's group with particularly high classification accuracies [43]. Early-stage LC discrimination from BPN has been reported by Haick et al. using an array of 40 chemiresistors based on MCNPs (Au NPs) and molecularlycoated SWCNTs, achieving an accuracy of $87 \%$. Considering that the required treatment may change in the occurrence of genetic alternations, the differentiation of patients with and without epidermal growth factor receptor (EGFR) mutation was also attempted with an accuracy of $83 \%$ [228]. 
Table 4. Sensing devices used for differential diagnosis, staging, and phenotyping of different categories of diseases.

\begin{tabular}{|c|c|c|c|c|c|}
\hline Sensor & Diseases/Phenotypes/Stages & Subjects & Classifier & Results & Ref. \\
\hline \multicolumn{6}{|c|}{ Differential diagnosis } \\
\hline \multirow{11}{*}{ Cyranose 320} & NSCLC vs. COPD (GOLD stage I-III) & 10 NSCLC, 10 COPD & PCA, CDA & $85 \%$ acc. & [223] \\
\hline & $\begin{array}{l}\text { LC vs. non-cancer (COPD, asthma, } \\
\text { pneumonia, pulmonary embolism, BPN) }\end{array}$ & $165 \mathrm{LC}, 91$ non-cancer & \multirow[t]{2}{*}{ SVM } & $87.3 \%$ acc. & \multirow[t]{2}{*}{ [224] } \\
\hline & LC vs. COPD vs. LC/COPD vs. HC & $63 \mathrm{LC}, 15 \mathrm{COPD}, 79$ both, $78 \mathrm{HC}$ & & $77.4 \%$ acc., $100 \%$ accurate LC/COPD classification & \\
\hline & $\begin{array}{l}\text { LC vs. non-cancer (COPD, asthma, } \\
\text { pneumonia, pulmonary embolism, } \\
\text { bronchiectasis, BPN, TB) }\end{array}$ & 252 LC, 223 non-cancer & LRA & $\begin{array}{l}\text { Sens.: } 95.8 \% \text { (S), } 96.2 \% \text { (NS) } \\
\text { Spec.: } 92.3 \% \text { (S) } 90.6 \% \text { (NS) }\end{array}$ & [225] \\
\hline & Asthma vs. COPD & 20 asthma, 30 COPD & PCA, CDA & $\begin{array}{c}96 \% \text { acc. } \\
\text { Within/between day repeatability, reproducibility of } \\
\text { e-Noses }\end{array}$ & [229] \\
\hline & \multirow{2}{*}{$\begin{array}{l}\text { Fixed and classic asthma vs. COPD } \\
\text { (GOLD stages II-III) }\end{array}$} & 21 fixed asthma, 40 COPD & \multirow{2}{*}{ PCA, CDA } & $88 \%$ acc., $85 \%$ sens., $90 \%$ spec. & \multirow{2}{*}[230]{} \\
\hline & & 39 classic asthma, 40 COPD & & $83 \%$ acc., $91 \%$ sens., $90 \%$ spec. & \\
\hline & IPF vs. COPD & $32 \mathrm{IPF}, 33 \mathrm{COPD}$ & PCA, CDA & $\begin{array}{l}80 \% \text { cross-validated acc., Wider patient cohorts and } \\
\text { inclusion of more comorbidities needed }\end{array}$ & [231] \\
\hline & COPD vs. LC vs. BC & $50 \mathrm{COPD}, 30 \mathrm{LC}, 50 \mathrm{BC}$ & PCA, CDA, CAP & $\begin{array}{c}\text { Correct classification values: } \\
\text { LC vs. COPD } 96.47 \% \text {, LC vs. BC } 93.05 \% \text {, BC vs. COPD } \\
100 \% \text {, COPD vs. LC vs. BC } 91.35 \%\end{array}$ & [201] \\
\hline & Bronchial vs. Laryngeal SCC (advanced) & 10 bronchial, 10 laryngeal & JMP Pro & $10 \%$ misclassification, $100 \%$ sens., $80 \%$ spec. & [232] \\
\hline & AD vs. PD vs. HC & $18 \mathrm{AD}, 16 \mathrm{PD}, 19 \mathrm{HC}$ & LDA & $76.2 \%$ sens., $45.8 \%$ spec., $p<0.0001$ & [233] \\
\hline \multirow{6}{*}{ AeoNose } & \multirow{3}{*}{$\begin{array}{l}\text { ILDs (COP, CTD) vs. COPD, } \\
\text { ILDs subgroups (COP, HP, IPF, } \\
\text { sarcoidosis, uILD, asbestosis, NSIP, } \\
\text { RB-ILD, DIP) }\end{array}$} & $28 \mathrm{COP}, 23 \mathrm{COPD}$ & \multirow{3}{*}{ Athena program, $t$-test } & AUC $0.77,75 \%$ sens., $71 \%$ spec. & \multirow{3}{*}[234]{} \\
\hline & & 25 CTD-ILD, 23 COPD & & AUC $0.85,88 \%$ sens., $71 \%$ spec. & \\
\hline & & 174 ILDs, 23 COPD & & $\begin{array}{l}\text { Less accurate discrimination of ILDs subgroups (e.g., } \\
\text { AUC IPF vs. CTD-ILD 0.86, COP vs. CTD-ILD 0.82) }\end{array}$ & \\
\hline & Asthma vs. CF & 20 asthma (moderate-severe), $13 \mathrm{CF}$ & ANN & AUC $0.90,89 \%$ sens., $77 \%$ spec. & [235] \\
\hline & HNSCC vs. LC & 52 HNSCC, 32 LC & ANN & $\begin{array}{l}\text { Acc., sens., spec.: } 93 \%, 96 \%, 88 \% \text { (best fit), } \\
85 \%, 85 \%, 84 \% \text { (cross-validation) }\end{array}$ & [236] \\
\hline & Cancer types & 100 HNSCC, 40 bladder, 28 colon cancer & ANN & $\begin{array}{c}\text { Acc., sens., spec.: } 81 \%, 79 \%, 81 \% \text { HNSCC vs. colon } \\
\text { cancer, } \\
84 \%, 80 \%, 86 \% \text { HNSCC vs. bladder cancer, } \\
84 \%, 88 \%, 79 \% \text { Colon vs. bladder cancer }\end{array}$ & [237] \\
\hline
\end{tabular}


Table 4. Cont.

\begin{tabular}{|c|c|c|c|c|c|}
\hline Sensor & Diseases/Phenotypes/Stages & Subjects & Classifier & Results & Ref. \\
\hline & LC vs. COPD vs. asthma vs. HC & 31 LC, 31 COPD, 37 asthma, 45 HC & PCA & Cross-validation values $78-88 \%$, repeatability $\uparrow$ & [91] \\
\hline SpiroNose & ILD subgroups: & $\begin{array}{c}141 \text { sarcoidosis, } 85 \text { IPF, } 33 \text { CTD-ILD, } 25 \\
\text { HP, } 11 \text { IPAF, } 10 \text { NSIP }\end{array}$ & PLS-DA & $\begin{array}{c}\text { Acc., sens., spec.: } \\
\text { 77\%, } 75 \% \text {, } 84 \% \text { IPF vs. HP, } 94 \%, 98 \%, 85 \% \text { IPF vs. } \\
\text { CTD-ILD, } 92 \%, 92 \%, 90 \% \text { IPF vs. NSIP, } 89 \%, 87 \%, 100 \% \\
\text { IPF vs. IPAF, 75\%, } 100 \% \text {, } 67 \% \text { CTD-ILD vs. IPAF, } 98 \% \text {, } \\
\text { 90\%, } 100 \% \text { CTC-ILD vs. NSIP, } 90 \%, 94 \%, 72 \% \text { HP vs. } \\
\text { sarcoidosis, } 91 \% \text {, } 92 \% \text {, } 88 \% \text { (training), } 91 \%, 95 \%, 79 \% \\
\text { (validation) IPF vs. non-IPF }\end{array}$ & [207] \\
\hline $\begin{array}{l}\text { Chemiresistor- } \\
\text { based } \\
\text { alkane sensor }\end{array}$ & $\begin{array}{l}\text { LC vs. HC } \\
\text { LC vs. COPD }\end{array}$ & $12 \mathrm{LC}, 12 \mathrm{COPD}, 13 \mathrm{HC}$ & MANOVA & $\begin{array}{l}\text { LC: } 83.3 \% \text { sens., } 88 \% \text { spec. } \\
\text { Sensor acc no smoke-dependence }\end{array}$ & [108] \\
\hline \multirow{2}{*}{$\begin{array}{l}\text { MOS, } \\
\text { electrochemical, hot } \\
\text { wire, and catalytic }\end{array}$} & LC vs. COPD & $48 \mathrm{LC}, 52 \mathrm{COPD}$ & 8 different & $\begin{array}{c}\text { 76.9-84.75\% acc., } 75-81.36 \% \text { sens., } 78.79-88.14 \text { spec. } \\
\text { Highest acc. With KPCA-XGBoost }\end{array}$ & [226] \\
\hline & LC vs. COPD & $33 \mathrm{LC}, 28 \mathrm{COPD}$ & $\begin{array}{c}\text { PCA-SVM, KPCA-SVM, } \\
\text { PCA-XGBoost, KPCA-XGBoost }\end{array}$ & $\begin{array}{c}\text { 82.52-96\% acc., } 78.33-95 \% \text { sens., } 85-96.67 \% \text { spec. } \\
\text { Highest acc. With KPCA-XGBoost }\end{array}$ & [227] \\
\hline \multirow{6}{*}{$\begin{array}{l}\text { Organically-coated } \\
\text { AuNPs and } \\
\text { SWCNTs based } \\
\text { chemiresistor }\end{array}$} & LC (I/II) vs. BPN & $16 \mathrm{LC}, 30 \mathrm{BPN}$ & DFA & $\begin{array}{l}87 \% \text { acc., } 75 \% \text { sens., } 93 \% \text { spec. } \\
\text { Low LC sample } \rightarrow \text { careful interpretation }\end{array}$ & [228] \\
\hline & $\mathrm{BC}$ vs. benign & $30 \mathrm{HC}, 15 \mathrm{BBT}, 13 \mathrm{DCIS}, 96 \mathrm{BC}$ & DFA & $\begin{array}{c}\text { Acc., sens., spec.: } 88.3 \%, 90.6 \%, 83.3 \% \text { BC vs. BBT } / H C, \\
71.2-82 \%, 62.6-80 \%, 75.7-82.3 \% \text { BC vs. BBT, } \\
81.4-84.4 \%, 83-83.3 \%, 81-92 \% \text { BC vs. DCIS }\end{array}$ & [238] \\
\hline & Gca vs. OLGIM groups (0-IV) & $\begin{array}{l}99 \text { Gca, } 155 \text { OLGIM 0, } 136 \text { OLGIM I-II, } \\
34 \text { OLGIM III-IV, } 53 \text { PUD }\end{array}$ & DFA & $\begin{array}{c}\text { Acc., sens., spec.: } \\
92 \%, 73 \%, 98 \% \text { Gca vs. } 0-I V, 84 \%, 90 \%, 80 \% \text { Gca vs. } 0, \\
87 \%, 97 \%, 84 \% \text { Gca vs. } 0-I I, 90 \%, 93 \%, 80 \% \text { Gca vs. III-IV, } \\
85 \%, 93 \%, 80 \% \text { Gca vs. I-IV, } 87 \%, 87 \%, 87 \% \text { Gca vs. PUD }\end{array}$ & [239] \\
\hline & Gca vs. benign gastric conditions & \multirow{2}{*}{$\begin{array}{l}37 \mathrm{Gca}, 32 \text { ulcers, } 61 \text { less severe } \\
\text { conditions }\end{array}$} & \multirow[t]{2}{*}{ DFA } & \multirow{2}{*}{$\begin{array}{l}89 \% \text { sens., } 90 \% \text { spec. } \\
84 \% \text { sens., } 87 \% \text { spec. }\end{array}$} & \multirow[t]{2}{*}[240]{} \\
\hline & ulcer vs. less severe & & & & \\
\hline & $\begin{array}{c}\text { AD vs. PD } \\
\text { AD vs. PD vs. HC }\end{array}$ & $15 \mathrm{AD}, 30 \mathrm{PD}, 12 \mathrm{HC}$ & DFA & $\begin{array}{c}\text { AD vs. PD: } 84 \% \text { acc., } 80 \% \text { sens., } 87 \% \text { spec. } \\
\text { Feasible overall discrimination, with large PD/HC } \\
\text { overlap }\end{array}$ & [114] \\
\hline NA-NOSE & $\begin{array}{l}\mathrm{BC} \text {, benign breast conditions, normal } \\
\text { mammographs }\end{array}$ & $\begin{array}{l}11 \mathrm{BC}, 14 \text { benign, } 7 \text { normal } \\
\text { mammographs }\end{array}$ & $\begin{array}{l}\text { PCA/ANOVA/Student's } t \text {-test, } \\
\text { SVM }\end{array}$ & $\begin{array}{l}94 \% \text { sens., } 80 \% \text { spec. for benign vs. BC and negative } \\
\text { mammography, Similar results with both methods }\end{array}$ & [116] \\
\hline \multirow{2}{*}{$\begin{array}{l}\text { MCNPs-based } \\
\text { chemiresistor- } \\
6 \text { sensors-array }\end{array}$} & IBD vs. IBS & \multirow{2}{*}{71 IBD (35 UC, 36 CD), 26 IBS } & \multirow{2}{*}{ ANN } & $\begin{array}{l}81 / 88 \% \text { acc., } 92 / 73 \% \text { sens., } 53 / 100 \% \text { spec. } \\
\text { (real/artificial) }\end{array}$ & \multirow{2}{*}{ [111] } \\
\hline & CD vs. UC & & & $\begin{array}{l}\text { 75/96\% acc., } 75 / 100 \% \text { sens., } 47 / 93 \% \text { spec. } \\
\text { (real/artificial) }\end{array}$ & \\
\hline
\end{tabular}


Table 4. Cont.

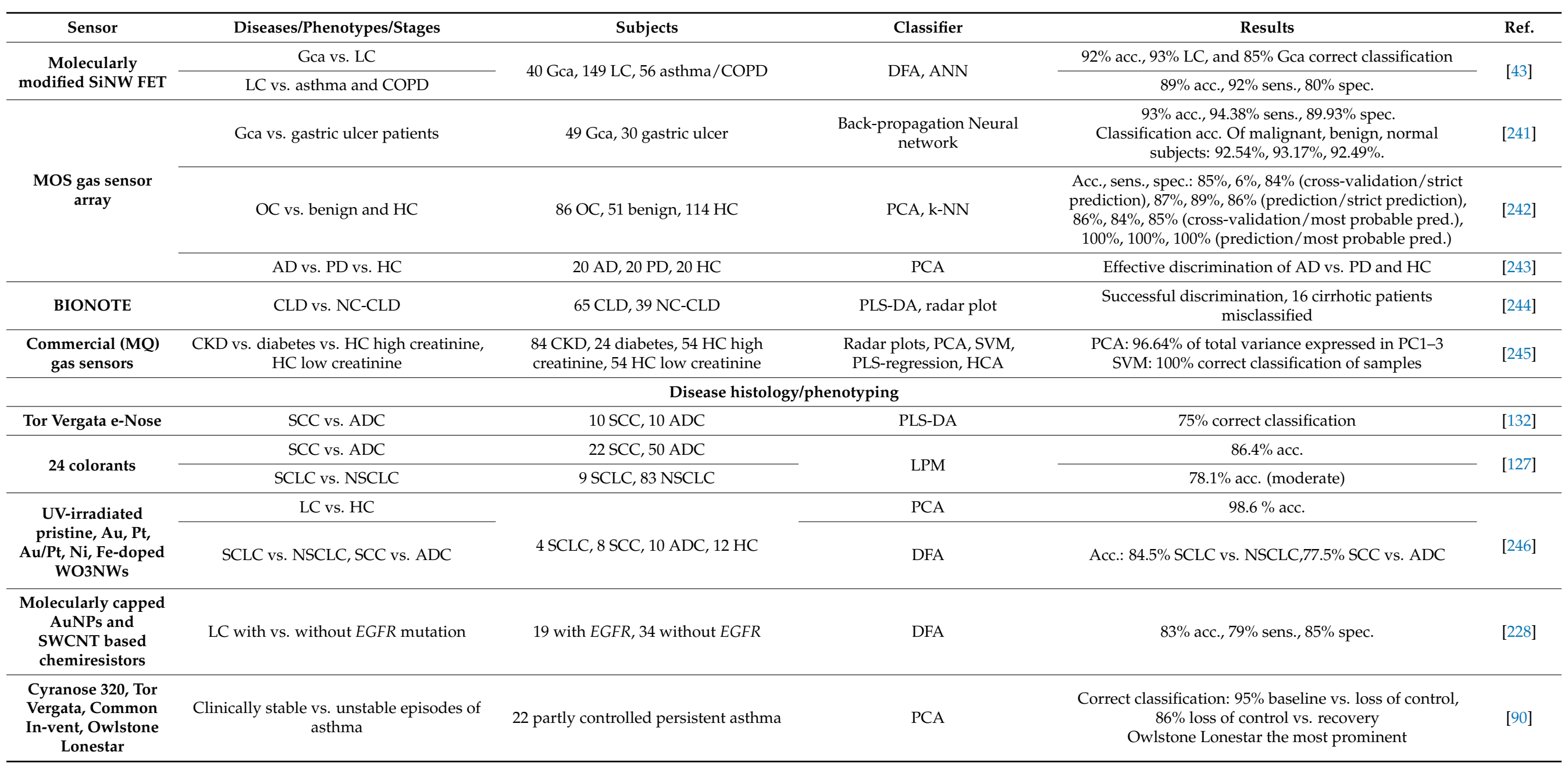


Table 4. Cont.

\begin{tabular}{|c|c|c|c|c|c|}
\hline Sensor & Diseases/Phenotypes/Stages & Subjects & Classifier & Results & Ref. \\
\hline \multirow{3}{*}{ Cyranose 320} & Asthma inflammatory phenotypes & 24 EOS., 10 NEUTR., 18 PAUC. & PCA & $\begin{array}{c}\text { Acc., sens., spec.: } 73 \%, 60 \%, 79 \% \text { EOS. vs. NEUTR., } 74 \% \text {, } \\
55 \%, 87 \% \text { EOS vs. PAUC., } 89 \%, 94 \%, 80 \% \text { NEUTR. vs. } \\
\text { PAUC. }\end{array}$ & [247] \\
\hline & Uncontrolled asthma-like symptoms & $\begin{array}{c}\text { Training set: } 65 \text { cluster } 1,22 \text { cluster 2, } 34 \\
\text { cluster } 3\end{array}$ & $\begin{array}{l}\text { one-way ANOVA, } \\
\text { Kruskal-Wallis }\end{array}$ & $\begin{array}{l}\text { Significant differences concerning chest tightness during } \\
\text { exercise, dyspnea and gender }\end{array}$ & [248] \\
\hline & $\begin{array}{l}\text { HC and controlled vs. partly controlled } \\
\text { and uncontrolled asthma }\end{array}$ & $\begin{array}{l}10 \mathrm{HC}, 9 \text { controlled, } 7 \text { partly, } 12 \\
\text { uncontrolled }\end{array}$ & PCA, radar plot & $\begin{array}{c}\text { Good predictive ability } \\
\text { Cross-validated AUC 0.80, 79\% sens., } 84 \% \text { spec. }\end{array}$ & [119] \\
\hline $\begin{array}{c}\text { Organically-coated } \\
\text { AuNPs and } \\
\text { SWCNT-based } \\
\text { chemiresistor }\end{array}$ & BC subtypes & $\begin{array}{l}12 \text { LuminalA, } 42 \text { LuminalB, } 12 \text { Triple } \\
\text { Negative, } 16 \text { HER2+, } 14 \text { HER2 } \\
\text { equivocal }\end{array}$ & DFA & 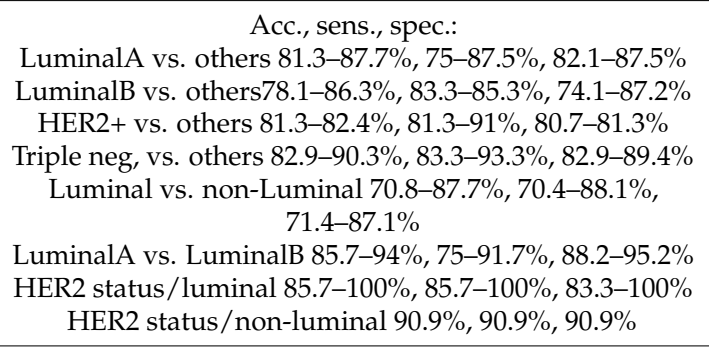 & [238] \\
\hline \multicolumn{6}{|c|}{ Disease staging } \\
\hline Tor Vergata e-Nose & LC Stage I vs. II/III/IV & 40 stage I, 18 stage II, 6 III/IV & PLS-DA & $\begin{array}{c}\text { Sens.: stage I } 90 \% \text { vs. stage II-IV 57\% (+ metabolic } \\
\text { diseases), } \\
\text { stage I } 96 \% \text { vs. stage II-IV 60\% (LC only) }\end{array}$ & [133] \\
\hline 24 colorants & LC Stage I/II vs. LC stage III/IV & 41 SCLC, 42 NSCLC & LPM & $79.3 \%$ acc. (moderate) & [127] \\
\hline $\begin{array}{l}11 \text { sensor-array } \\
\text { (MOS, } \\
\text { electrochemical, hot } \\
\text { wire and catalytic) }\end{array}$ & LC Stage III vs. IV & 44 stage II, 46 stage IV & $\begin{array}{c}\text { PCA-SVM, KPCA-SVM, } \\
\text { PCA-XGBoost, KPCA-XGBoost }\end{array}$ & $70.42-82.42 \%$ acc., $45-81 \%$ sens., $79-95.5 \%$ spec. & [227] \\
\hline \multirow{2}{*}{$\begin{array}{l}\text { Organically-coated } \\
\text { AuNPs and } \\
\text { SWCNTs based } \\
\text { chemiresistor }\end{array}$} & OLGIM stages & $\begin{array}{l}155 \text { OLGIM 0, } 136 \text { OLGIM I-II, } 34 \\
\text { OLGIM III-IV, } 7 \text { Dysplasia }\end{array}$ & DFA & $\begin{array}{l}\text { Acc., sens., spec.: } 0 \text {-II vs. III-IV and dysplasia } 61 \%, 83 \% \text {, } \\
60 \%, 0 \text { vs. I-II } 43 \%, 45 \%, 41 \% \text {, } 0 \text { vs. III-IV } 66 \%, 90 \%, 61 \% \text {, } \\
0 \text { vs. I-IV } 50 \%, 50 \%, 50 \% \text {, I-II vs. III-IV } 64 \%, 80 \%, 60 \%\end{array}$ & [239] \\
\hline & GCa I-II vs. III-IV & 17 GCa I-II, 18 GCa III-IV & DFA & $89 \%$ sens., $94 \%$ spec. & [240] \\
\hline \multirow{2}{*}{$\begin{array}{c}\text { Molecularly } \\
\text { modified SiNW FET }\end{array}$} & LC staging (I-II vs. III-IV) & \multirow{2}{*}{34 early stage, 110 advanced stage } & \multirow{2}{*}{ DFA, ANN } & $81 \%$ acc., $34.5 \%$ sens., $95 \%$ spec. & \multirow{2}{*}[43]{} \\
\hline & GCa staging (I-II vs. III-IV) & & & $\begin{array}{c}\text { 86.5\% correct classification, } 84.6 \text { early stage, } 87.5 \\
\text { advanced }\end{array}$ & \\
\hline
\end{tabular}


Table 4. Cont.

Sensor

Cyranose 320

BIONOTE
Diseases/Phenotypes/Stages
Subjects

bronchial: 10 in situ, 10 advanced

NA
Classifier

JMP Pro

Results

Ref

PLS-DA

Liver cirrhosis (A, B, C Child-Pugh)

Acc.: accuracy, AD: Alzheimer's disease, ADC: adenocarcinoma, AUC: Area Under the Receiver Operating Characteristic Curve, ANN: artificial neural network, BC: breast cancer, BBT Breast benign tumor, BPN: benign pulmonary nodules, CAP: canonical analysis of principal coordinates, CD: Crohn's Disease, CDA: canonical discriminant analysis, CF: cystic fibrosis, CLD: chronic liver disease, COP: cryptogenic organizing pneumonia, COPD: chronic obstructive pulmonary disease, CTD-ILD: connective-tissue diseases-associated ILD, DCIS: ductal carcinoma in situ, DFA: Discriminant Function Analysis, DIP: desquamative interstitial pneumonia, EOS: eosinophilic, GCa: gastric cancer, GOLD: Global Initiative for Obstructive Lung Disease, HBC: hexabenzocoronene, HCA: Hierarchical Cluster Analysis, HER2: Human epidermal growth factor receptor 2, HNSCC: Head and neck squamous cell carcinoma, HP: hypersensitivity pneumonitis, IBD: Inflammatory Bowel Diseases, IBS: Irritable Bowel Syndrome, ILDs: interstitial lung diseases, IPAF: interstitial pneumonia with autoimmune features, IPF: idiopathic pulmonary fibrosis, k-NN: k-Nearest Neighbors, KPCA-XGBoost: kernel principal component analysis—extreme gradient boosting, LC: Lung cancer, LRA: logistic regression analysis, LPM: Logistic prediction model, MANOVA: multivariate analysis of variance, MOS: metal oxide semiconductor, NC-CLD: non-cirrhotic CLD, NEUTR: neutrophilic, NS: non-smokers, NSCLC: non-small cell lung cancer, NSIP: non-specific interstitial pneumonia, NWs: nanowires, OC: ovarian cancer, OLGIM: operative link on gastric intestinal metaplasia, PAH: Polycyclic aromatic hydrocarbons, PAUC: paucigranulocytic, PCA: Principal Component Analysis, PD: Parkinson's disease, PLS-DA: Partial Least Square Discriminant Analysis, PUD: peptic ulcer disease, RB-ILD: respiratory bronchiolitis-associated ILD, S: smokers, SCC: squamous cell carcinoma, SCLC: small cell lung cancer, Sens.: sensitivity, Spec. specificity, SVM: Support Vector Machine, TB: Tuberculosis, UC: Ulcerative Colitis, uILD: unclassifiable ILD, UV: ultra-violet. 

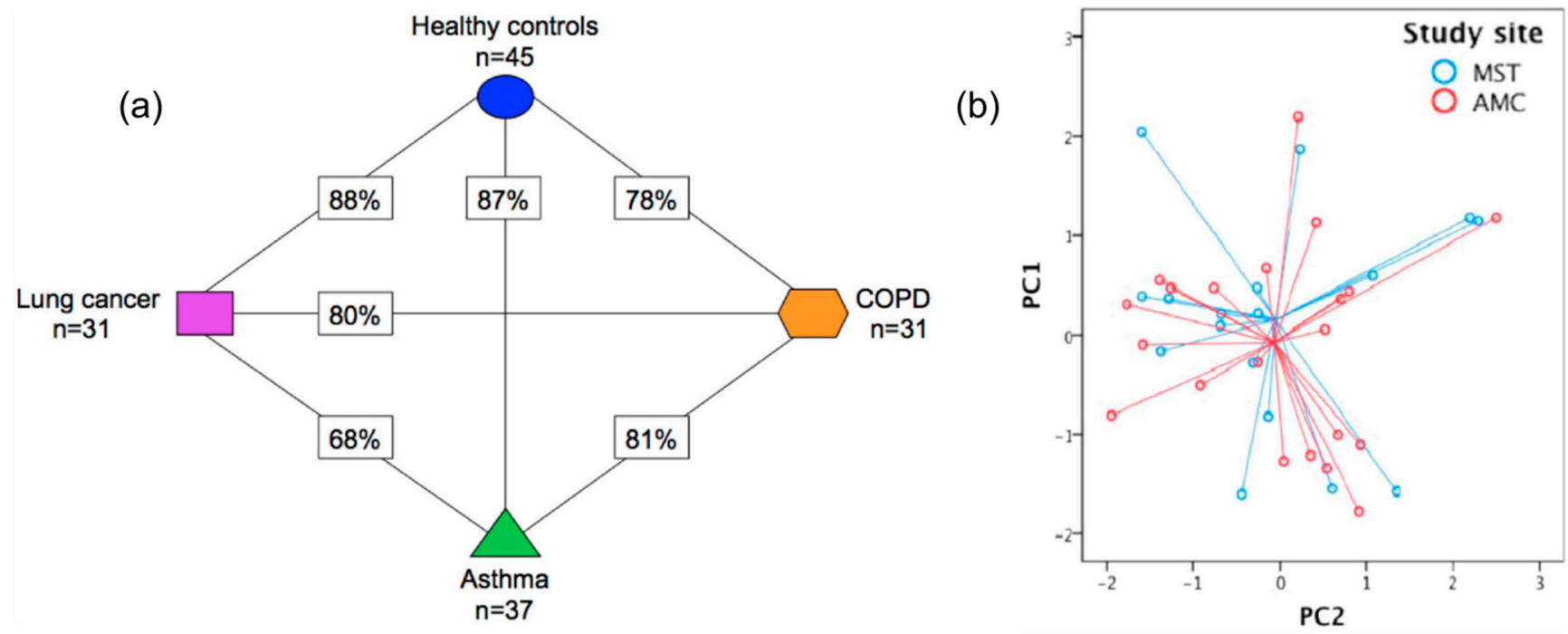

Figure 14. (a) Presentation of the cross-validation percentages of the differentiation of asthma, COPD, LC patients, and HC, using SpiroNose; (b) PCA plot of breathprints collected from asthmatic patients at the Academic Medical Center (AMC), Amsterdam and Medical Spectrum Twente (MST), Enschede, for which no significant differentiation is observed $(p=0.892)$. Adapted with permission from Ref. [91]. Copyright @ 2015 IOP Publishing Ltd.

Concerning LC histology and staging with sensing devices, promising studies have been reported in the literature. The discrimination of NSCLC subtypes ADC and SCC has been permitted using Tor Vergata e-Nose with an accuracy of 75\%, by applying endoscopic breath sampling [132] as well as by using a colorimetric sensor-array of 24 elements developed by Mazzone et al. ultimately achieving an accuracy of $86.4 \%$ [127]. SCLC and NSCLC differentiation and LC staging (I/II vs. III/IV) were also examined by Mazzone et al. though with moderate accuracies [127]. A 6-sensor-array based on UV-irradiated (394 nm) pristine or metal-doped $\mathrm{WO}_{3} \mathrm{NWs}$ (Table 4) differentiated effectively not only ADC from SCC, but also between SCLC and NSCLC with $77.5 \%$ and $84.5 \%$ accuracy values, respectively (Figure 15) [246]. In another study aiming at the discrimination of LC patients from $\mathrm{HC}$ while taking into account the existence of metabolic comorbidities, Tor Vergata e-Nose exhibited far higher sensitivity for stage I LC in comparison to the rest of stages, either in the presence or absence of metabolic diseases (Table 4) [133]. LC staging was recently attempted by Liu et al. along with COPD discrimination as mentioned above, with stage III LC being effectively discriminated from stage IV with an accuracy higher than $80 \%$, using KPCA-XGBoost [227]. Haick's team has achieved LC staging with an accuracy of $81 \%$ and with low sensitivity, using a molecularly modified Si NW FET (Table 4) [43].

As in the case of breath analysis with analytical methods, precise diagnosis of lung diseases other than LC via sensing devices is an extensive field of research. The effective discrimination of COPD and asthma has been reported in the literature by Fens at al. using Cyranose 320 and taking into consideration smoking habits, leading to high cross-validated accuracy values (Table 4) $[229,230]$. More recently, asthma and CF discrimination was also reported for pediatric population using AeoNose and with high accuracy values, excluding the confounding factors of diet, exercise, comorbidities and inhaled drugs [235]. Concerning ILDs, Krauss et al. used the AeoNose in an attempt to differentiate between ILDs subgroups (Table 4), with moderate accuracy, as well as between ILDs cryptogenic organizing pneumonia and connective-tissue diseases-associated ILD from COPD patients with good sensitivity and specificity [234]. COPD and IPF differentiation has been recently investigated by Dragonieri et al., with a high accuracy of $80 \%$, verified by external validation using Cyranose 320 [231]. In contrast to Krauss et al., Moor's group achieved to reliably discriminate patients suffering from different ILDs by using SpiroNose as well as greater cohorts of ILD-patients (Table 4) [207]; the group demonstrated the applicability of e-Noses 
in ILDs differential diagnosis and specifically in IPF discrimination from non-IPF patients with high accuracies (91\%) [207].
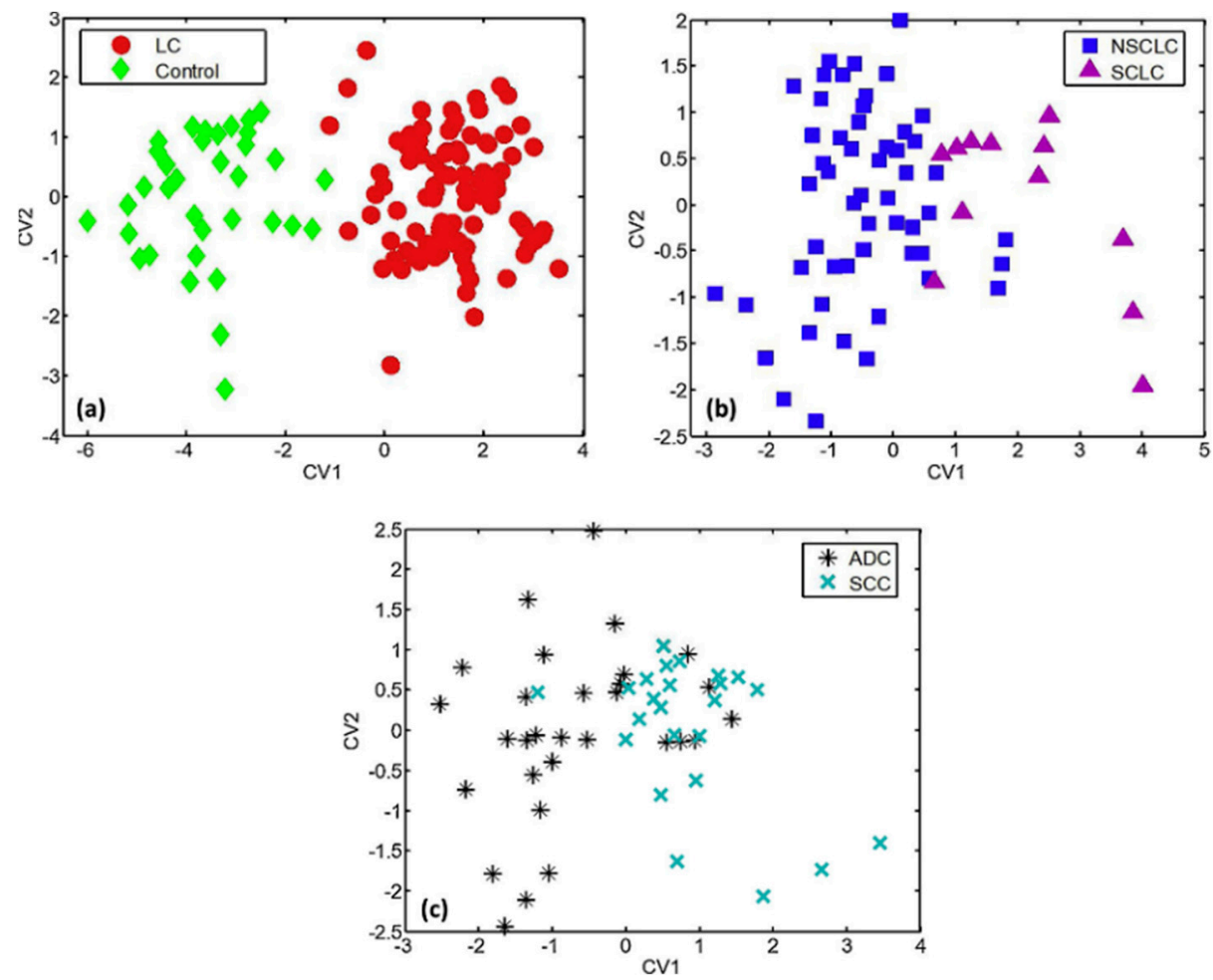

Figure 15. DFA plots representing the discrimination of (a) LC patients from HC; (b) SCLC from NSCLC patients; and (c) SCC from ADC patients, using a 6-sensor array of UV-irradiated (394 nm) pristine or metal-doped $\mathrm{WO}_{3} \mathrm{NWs}$. The arrays achieved the detection of lung cancer but also the prediction of LC histological subtypes. Reprinted with permission from Ref. [246]. Copyright @ 2020 Published by Elsevier B.V.

Disease phenotyping using sensing devices seems to be also feasible. Plaza et al. achieved differentiation between the three inflammatory phenotypes of asthma with high accuracy values (Table 4), with the participants' phenotypes being characterized by differential leukocyte counts in induced sputum [247] while asthma-control assessment has been also reported. Brinkman et al. used 4 different e-Noses in order to discriminate between stable and unstable periods, comparing baseline (control) vs. loss of control and loss of control vs. recovery breath samples, with the Owlstone Lonestar being the most prominent concerning the discrimination of unstable periods (Table 4) [90]. More recently, Moreira et al. demonstrated the ability of Cyranose 320 to discriminate the uncontrolled asthma-like symptoms, using 3 different groups of asthmatic or suspicious of asthma participants divided by unsupervised hierarchical clustering [248]. The division of participants was based on asthma, lung function, symptoms of the last month, age, and food/drink intake $2 \mathrm{~h}$ before breath sampling [248]. In another recent study, the same e-Nose was used for the effective discrimination of $\mathrm{HC}$ and asymptomatic-controlled asthmatic children from the symptomatic partly-controlled and uncontrolled asthmatic children, after assessing the discriminatory ability of subsets of the 32 sensors of Cyranose 320 for the six different possible combinations of the 4 studied groups; increased feasibility and modest to good diagnostic accuracy values were obtained [119]. Cyranose 320 has been also used for COPD phenotyping permitting (especially in the case of GOLD stage I) the detection of activation of inflammatory cells, indicating increased inflammatory activity in mild rather than severe COPD [120]. 


\subsection{Cancers}

Discrimination between different cancer types and cancer stages/histologies as well as between malignant and benign tumors (additionally to LC which has been mentioned earlier) using breath analysis of VOCs is also a hot research topic. Analytical techniques have been used for such applications. Phillips at al., for example, detected 5 VOCs and were able to differentiate $\mathrm{BC}$ patients from patients with abnormal mammograms and negative biopsies, with $93.8 \%$ sensitivity and $84.6 \%$ specificity [249]. Haick's group identified 21 exhaled VOCs that were significantly different between HC and patients suffering from breast benign tumors (BBT), ductal carcinoma in situ (DCIS, early-stage BC) and $\mathrm{BC}$; a potentially cancer-related set of 14 VOCs that were significantly different between malignant and non-malignant patients was also identified in this study thus permitting group differentiation with $78 \%$ sensitivity and $72 \%$ accuracy [238]. In another study by the same group, the detection of GCa and the presence/absence and risk level of precancerous lesions was attempted using GC-MS so as to identify 8 VOCs statistically different between GCa and operative link on gastric intestinal metaplasia (OLGIM) groups (e.g., GCa vs. OLGIM 0-IV, GCa vs. OLGIM 0-II, GCa vs. OLGIM 0), as well as between GCa and peptic ulcer disease (PUD) and OLGIM 0-IV and PUD ( $p$-values < 0.017) [239]. Those 8 VOCs, in different combinations, are considered to correspond to the breathprints of OLGIM groups [239].

Remarkably, respective applications of sensing devices have been extensively investigated for various cancer types. Haick's group has used NA-NOSE in order to discriminate between subjects with $\mathrm{BC}$, benign breast conditions or normal mammographs, achieving increased sensitivity and specificity values for the BBT patients in comparison to the other 2 groups [116]. In another study by the same group a chemiresistor based on organicallycoated Au NPs and SWCNTs was successfully used for the differentiation of BC from BBT and HC, BBT only or DCIS only, as well as for the differentiation of different molecular BC sub-groups as presented on Table 4. Larger studies are necessary, though for significant statistical results and more information to be obtained [238]. Very recently, differentiation between $\mathrm{BC}$ and $\mathrm{LC}$ has been achieved as well by Rodriguez et al. with a correct classification of $93.05 \%$ [201]. Concerning GCa, the differentiation of GCa and OLGIM groups as well as between different OLGIM stages (e.g., OLGIM 0 vs. I-II, 0 vs. I-IV, I-II vs. III-IV) has been attempted by Haick's group using the same type of chemiresistor-array and leading to high validated accuracy values in some cases (Table 4) [239]. In another promising study, Haick et al. used a nanomaterial-based chemiresistor (Table 4) that permitted the successful discrimination of Gca from benign conditions along with Gca staging (early vs. late stages) [240]. Gca differentiation from gastric ulcer patients has been achieved by Daniel et al. with a great classification ability, using an array of commercial MOS gas sensors and various ANN types [241]. Remarkably, a molecularly modified Si NW FET, developed by Haick's group, has permitted Gca staging with an accuracy of $87 \%$ as well as Gca differentiation from LC with an accuracy of 92\% [43]. More recently, discrimination of ovarian cancer (OC) from women with benign tumors and HC was achieved by Raspagliesi et al. with great classification performance, both in the case of strict and most probable prediction, using again MOS sensors [242]. Notably in class prediction application, 4/23 early-stage OC patients were misclassified as benign/HC along with 2/14 OC patients with tumor size $<3 \mathrm{~cm}$ in cross validation phase, while in prediction phase only $1 / 9$ early-stage patients were misdiagnosed [242]. Another common cancer type, i.e., head and neck cancer (HNC), has been studied for differential diagnosis with sensing devices. As an example, Hooren et al. attempted to discriminate HNC from LC with a high accuracy of $93 \%$ analyzing patients' exhaled breath with AeoNose, excluding cutaneous tumors and salivary glands malignancies [236]. HNC differentiation from colon and bladder cancer with AeoNose was also reported, by the same group along with the discrimination between bladder and colon cancer, demonstrating the discriminant ability of the e-Nose for those cancer types after double cross-validation [237]. More recently, Cyranose 320 was used for the differentiation of advanced bronchial (LC) and laryngeal (HNC) SCC, as well as for the 
discrimination of advanced and in situ stages of bronchial and laryngeal SCC, leading to successful classification of the groups (Table 4) [232].

\subsection{Liver, Renal, and Intestinal Diseases}

Liver cirrhosis, chronic hepatitis [244], CKD [113], and inflammatory bowel diseases (IBD) comprise common liver, kidney, and intestinal diseases, respectively. As far as CKD is concerned, it is characterized by gradual loss of kidney function within months or years, while different treatment is demanded depending on disease stage (stages I-V) [113]. Similarly, in the case of chronic liver disease (CLD), disease staging is of great importance; following CLD diagnosis, using invasive biopsy, liver function assessment is conducted biochemically [244]. On the other hand, early stage and precise IBD and IBS diagnosis, as well as the invasive diagnostic methods followed for IBD, comprise challenging issues [111]. Consequently, precise diagnosis and staging of liver, kidney and intestinal diseases are particularly important and have been attempted using breath analysis with sensing devices. Pennazza et al. for instance used BIONOTE e-nose to successfully differentiate not only liver cirrhosis and CLD from non-cirrhotic CLD (chronic hepatitis) but also liver cirrhosis stages by taking into account smoking habits and potential comorbidities (e.g., diabetes, lung, and heart diseases) [244]. Concerning renal diseases, Haick's group achieved CKD staging using organically functionalized Au NPs-based chemiresistors and SVM [113]. The classification of CKD stage IV in relation to stage $\mathrm{V}$ was permitted by 2 or 3 sensors with an accuracy of $85 \%$, a sensitivity of $75 \%$ and specificity of $92 \%$ while only one sensor allowed for the discrimination of early and advanced stages with 76\% accuracy, 75\% sensitivity, and 77\% specificity [113]. The discrimination of CKD from other diseases has been also attempted. Specifically, discrimination of CKD, diabetes, and HC with high or low creatinine has been attempted with success using an array of commercial (MQ) sensors along with different classification methods, with SVM and PCA leading to good group classification [245]. Remarkably, pre-concentration or dehumidification were not needed for clear classification to be accomplished [245]. The effective discrimination between the intestinal diseases IBD and IBS has been also reported along with further differentiation of the IBD into ulcerative colitis (UC) and Crohn's disease (CD), using not only artificial but also real-breath samples and a MCNPs-based chemiresistor (Table 4) [111]. The higher accuracy values observed when using artificial samples is expected and attributed to the standard concentration of VOCs, contrary to the variable concentration of VOCs in breath [111].

\subsection{Neurodegenerative Diseases}

Neurodegenerative diseases are characterized by gradually augmented occurrence, as a direct consequence of the increased lifespan of human population [250], with Alzheimer (AD) and Parkinson (PD) being the most frequent [114]. Concerning AD, early disease detection is of great importance for preventing, decelerating and terminating the disease [250] while diagnosis for both diseases is based on the assessment of clinical symptoms [114]. Remarkably, the analysis of exhaled VOCs has been investigated for precise AD diagnosis as well as for differential diagnosis between AD and PD [114,250]. Recently, Tiele et al. attempted to discriminate mild cognitive impairment due to AD (MCI) from AD, using GC-MS, achieving $60 \%$ sensitivity and $84 \%$ specificity along with the detection of 6 potential discriminant VOCs [250]. Haick's group, on the other hand, achieved the discrimination of $\mathrm{AD}$ and PD as well as an overall discrimination of $\mathrm{AD}, \mathrm{PD}$, and HC, using an array of 20 nanomaterial-based chemiresistors and GC-MS (Table 4) [114]. In a similar study, the ability of IMS and Cyranose 320 to differentiate AD, PD, and HC was demonstrated, achieving a high overall discriminant capability (Table 4) [233]. IMS analysis revealed five VOCs significantly different between the groups [233]. The discrimination of the same groups of subjects has been also attempted using different arrays of MOS sensors (TGS, MICS) with one of the combinations (8 MOS sensors) demonstrating the best discriminant ability [243]. 


\section{Conclusions and Future Perspectives}

Exhaled breath analysis, especially using selective or cross-reactive sensors, comprises a non-invasive method that holds a great promise for application in early-stage and differential diagnosis of not only respiratory but also systemic diseases. The aim of this review was to present the main categories of nanomaterials and sensors that have been used up to now in exhaled breath analysis for disease diagnosis as well as to demonstrate the applicability of breath analysis in differential diagnosis, phenotyping, and staging of several types of diseases, especially via the use of cross-reactive sensing devices.

The progressive development of novel nanomaterials offers a great opportunity to develop more effective sensing elements, both for selective and cross-reactive sensors and especially for point-of-care diagnosis, treatment monitoring and population screening. However, fundamental challenges in this novel research field inhibit the application of breath analysis in clinical practice and should therefore be addressed. Concerning analytical techniques used for exhaled VOCs identification, the use of bulky, expensive, and complex analytical devices is limited in hospitals while their incorporation in portable point-of-care systems is still unattainable [251]. In addition, the validity of breath analysis results is of major concern since the trace levels of exhaled VOCs affect the analysis accuracy [252]. At the same time the lack of clear breath sampling protocols [252], e.g., breath collection [251] and breath storage, could potentially change sample composition [251] and therefore emerge as important challenges. Sample composition can be also affected by confounding factors, i.e., age, gender, place of living, habits, and nutrition. In the case of sensors exhalation rate, a hardly controlled parameter, may also play a confusing role hence complicating the procedure [251].

For those limitations to be overcome, breath analysis research should focus on sampling and procedure protocols standardization $[118,251]$ and system improvement towards technical/physiological/pathophysiological confounders [118], in order to determine the endogenous VOCs and to define valid exhaled patterns of biomarkers. Towards this direction, standard correlations between blood and breath VOC concentrations could be established [251]. Furthermore, the development of portable/wearable and low-cost nanomaterial-based sensors that are resistant to humidity [251] and serve the clinical needs (i.e., selectivity for disease-specific VOCs and inorganic gases, small recovery time for population screening), along with the optimization of sensor training and validation by using different subject-groups comprise critical steps for the development of sensors applicable in clinical diagnosis [118]. Notably, the ability of new sensing systems to discriminate different diseases, to achieve precise early diagnosis of diseases with similar symptoms and underlying mechanisms, is a major concern [200] that has only recently been considered.

In order to meet all of the aforementioned goals, interdisciplinary research and cooperation is an essential prerequisite [251]. Breath analysis poses as a powerful and promising diagnostic tool that could eventually be used in clinical practice or in portable and compact health monitoring systems, provided it meets most of the existing challenges.

Author Contributions: Conceptualization, M.K., E.S. and D.T.; methodology, M.K., E.S. and D.T.; investigation, M.K.; writing-original draft preparation, M.K.; writing—review and editing, E.S.; supervision, E.S. and D.T.; project administration D.T., E.E. and N.L. All authors have read and agreed to the published version of the manuscript.

Funding: This research received no external funding.

Data Availability Statement: Not applicable.

Conflicts of Interest: The authors declare no conflict of interest. 


\section{References}

1. Chen, T.; Liu, T.; Li, T.; Zhao, H.; Chen, Q. Exhaled breath analysis in disease detection. Clin. Chim. Acta 2021, 515, 61-72. [CrossRef] [PubMed]

2. Gashimova, E.; Temerdashev, A.; Porkhanov, V.; Polyakov, I.; Perunov, D.; Azaryan, A.; Dmitrieva, E. Investigation of different approaches for exhaled breath and tumor tissue analyses to identify lung cancer biomarkers. Heliyon 2020, 6, e04224. [CrossRef] [PubMed]

3. Phillips, M.; Cataneo, R.N.; Saunders, C.; Hope, P.; Schmitt, P.; Wai, J. Volatile biomarkers in the breath of women with breast cancer. J. Breath Res. 2010, 4, 026003. [CrossRef] [PubMed]

4. Oakley-Girvan, I.; Davis, S.W. Breath based volatile organic compounds in the detection of breast, lung, and colorectal cancers: A systematic review. Cancer Biomarkers 2017, 21, 29-39. [CrossRef]

5. Pite, H.; Morais-Almeida, M.; Rocha, S.M. Metabolomics in asthma: Where do we stand? Curr. Opin. Pulm. Med. 2018, 24, 94-103. [CrossRef] [PubMed]

6. Vishinkin, R.; Haick, H. Nanoscale Sensor Technologies for Disease Detection via Volatolomics. Small 2015, 11, 6142-6164. [CrossRef]

7. Broza, Y.Y.; Vishinkin, R.; Barash, O.; Nakhleh, M.K.; Haick, H. Synergy between nanomaterials and volatile organic compounds for non-invasive medical evaluation. Chem. Soc. Rev. 2018, 47, 4781-4859. [CrossRef]

8. Tisch, U.; Haick, H. Chemical sensors for breath gas analysis: The latest developments at the Breath Analysis Summit 2013 J. Breath Res. 2014, 8, 027103. [CrossRef]

9. Song, G.; Qin, T.; Liu, H.; Xu, G.B.; Pan, Y.Y.; Xiong, F.X.; Gu, K.S.; Sun, G.P.; Chen, Z.D. Quantitative breath analysis of volatile organic compounds of lung cancer patients. Lung Cancer 2010, 67, 227-231. [CrossRef]

10. Ma, H.; Li, X.; Chen, J.; Wang, H.; Cheng, T.; Chen, K.; Xu, S. Analysis of human breath samples of lung cancer patients and healthy controls with solid-phase microextraction (SPME) and flow-modulated comprehensive two-dimensional gas chromatography (GC $\times$ GC). Anal. Methods 2014, 6, 6841-6849. [CrossRef]

11. Hakim, M.; Broza, Y.Y.; Barash, O.; Peled, N.; Phillips, M.; Amann, A.; Haick, H. Volatile organic compounds of lung cancer and possible biochemical pathways. Chem. Rev. 2012, 112, 5949-5966. [CrossRef]

12. Wilson, A.D. Advances in electronic-nose technologies for the detection of volatile biomarker metabolites in the human breath. Metabolites 2015, 5, 140-163. [CrossRef]

13. Bag, A.; Lee, N.E. Recent Advancements in Development of Wearable Gas Sensors. Adv. Mater. Technol. 2021, 6, 2000883. [CrossRef]

14. Bos, L.D.; Sterk, P.J.; Fowler, S.J. Breathomics in the setting of asthma and chronic obstructive pulmonary disease. J. Allergy Clin. Immunol. 2016, 138, 970-976. [CrossRef]

15. Broza, Y.Y.; Haick, H. Nanomaterial-based sensors for detection of disease by volatile organic compounds. Nanomedicine 2013, 8 , 785-806. [CrossRef]

16. Peng, G.; Hakim, M.; Broza, Y.Y.; Billan, S.; Abdah-Bortnyak, R.; Kuten, A.; Tisch, U.; Haick, H. Detection of lung, breast, colorectal, and prostate cancers from exhaled breath using a single array of nanosensors. Br. J. Cancer 2010, 103, 542-551. [CrossRef] [PubMed]

17. Fens, N.; van der Schee, M.P.; Brinkman, P.; Sterk, P.J. Exhaled breath analysis by electronic nose in airways disease. Established issues and key questions. Clin. Exp. Allergy 2013, 43, 705-715. [CrossRef]

18. Wang, M.; Sheng, J.; Wu, Q.; Zou, Y.; Hu, Y.; Ying, K.; Wan, H.; Wang, P. Confounding effect of benign pulmonary diseases in selecting volatile organic compounds as markers of lung cancer. J. Breath Res. 2018, 12, 046013. [CrossRef]

19. Hashoul, D.; Haick, H. Sensors for detecting pulmonary diseases from exhaled breath. Eur. Respir. Rev. 2019, 28, 190011. [CrossRef]

20. Meyer, N.; Dallinga, J.W.; Nuss, S.J.; Moonen, E.J.C.; van Berkel, J.J.B.N.; Akdis, C.; van Schooten, F.J.; Menz, G. Defining adult asthma endotypes by clinical features and patterns of volatile organic compounds in exhaled air. Respir. Res. 2014, 15, 136. [CrossRef] [PubMed]

21. Corradi, M.; Poli, D.; Bouza, M. Observation of nonanoic acid and aldehydes in exhaled breath of patients with lung cancer Observation of nonanoic acid and aldehydes in exhaled breath of patients with lung cancer. J. Breath Res. 2017, $11,26004$. [CrossRef]

22. Boots, A.W.; Van Berkel, J.J.B.N.; Dallinga, J.W.; Smolinska, A.; Wouters, E.F.; Van Schooten, F.J. The versatile use of exhaled volatile organic compounds in human health and disease. J. Breath Res. 2012, 6, 027108. [CrossRef]

23. Haick, H.; Cohen-Kaminsky, S. Detecting lung infections in breathprints: Empty promise or next generation diagnosis of infections. Eur. Respir. J. 2015, 45, 21-24. [CrossRef] [PubMed]

24. Shan, B.; Broza, Y.Y.; Li, W.; Wang, Y.; Wu, S.; Liu, Z.; Wang, J.; Gui, S.; Wang, L.; Zhang, Z.; et al. Multiplexed Nanomaterial-Based Sensor Array for Detection of COVID-19 in Exhaled Breath. ACS Nano 2020, 14, 12125-12132. [CrossRef] [PubMed]

25. Giovannini, G.; Haick, H.; Garoli, D. Detecting COVID-19 from Breath: A Game Changer for a Big Challenge. ACS Sens. 2021, 6, 1408-1417. [CrossRef]

26. Grassin-Delyle, S.; Roquencourt, C.; Moine, P.; Saffroy, G.; Carn, S.; Heming, N.; Fleuriet, J.; Salvator, H.; Naline, E.; Couderc, L.J.; et al. Metabolomics of exhaled breath in critically ill COVID-19 patients: A pilot study. EBioMedicine 2021, 63, 103154. [CrossRef] 
27. BreFenceTM Go COVID-19 Breath Test System Rapid Breath Test for COVID-19 Detection. Available online: https: //secureservercdn.net/160.153.138.177/8g1.9f8.myftpupload.com/wp-content/uploads/2021/05/Breathonix-BreFence -Go-Breath-Test-System.pdf (accessed on 25 November 2021).

28. HSA Grants Provisional Authorisation for 'BreFence Go COVID-19 Breath Test System' and 'Traciex Breathalyser' for Detection of COVID-19 Infection. Available online: https:/ / www.hsa.gov.sg/announcements/regulatory-updates/hsa-grants-provisional-a uthorisation-for-brefence-go-covid-19-breath-test-system-and-traciex-breathalyser-for-detection-of-covid-19-infection (accessed on 25 November 2021).

29. Provisional Authorisation for COVID-19 Tests-Breathonix Pte Ltd. Available online: https://www.hsa.gov.sg/docs/hprg-mdb /breathonix_brefence-go-covid-19-breath-test-system_provisional-authorisation-for-covid-19-tests_19052021.pdf (accessed on 25 November 2021).

30. Wang, C.; Sun, B.; Guo, L.; Wang, X.; Ke, C.; Liu, S.; Zhao, W.; Luo, S.; Guo, Z.; Zhang, Y.; et al. Volatile organic metabolites identify patients with breast cancer, cyclomastopathy, and mammary gland fibroma. Sci. Rep. 2014, 4, 5383. [CrossRef]

31. Pereira, J.; Porto-Figueira, P.; Cavaco, C.; Taunk, K.; Rapole, S.; Dhakne, R.; Nagarajaram, H.; Câmara, J.S. Breath analysis as a potential and non-invasive frontier in disease diagnosis: An overview. Metabolites 2015, 5, 3-55. [CrossRef]

32. Gaida, A.; Holz, O.; Nell, C.; Schuchardt, S.; Lavae-Mokhtari, B.; Kruse, L.; Boas, U.; Langejuergen, J.; Allers, M.; Zimmermann, S.; et al. A dual center study to compare breath volatile organic compounds from smokers and non-smokers with and without COPD. J. Breath Res. 2016, 10, 026006. [CrossRef]

33. Neerincx, A.H.; Vijverberg, S.J.H.; Bos, L.D.J.; Brinkman, P.; van der Schee, M.P.; de Vries, R.; Sterk, P.J.; Maitland-van der Zee, A.H. Breathomics from exhaled volatile organic compounds in pediatric asthma. Pediatr. Pulmonol. 2017, 52, 1616-1627. [CrossRef]

34. Amal, H.; Haick, H. Point of care breath analysis systems. In Advanced Nanomaterials for Inexpensive Gas Microsensors: Synthesis, Integration and Applications, 1st ed.; Llobet, E., Ed.; Elsevier Inc.: Amsterdam, The Netherlands; Oxford, UK; Cambridge, MA, USA, 2019; pp. 315-334.

35. Basanta, M.; Jarvis, R.M.; Xu, Y.; Blackburn, G.; Tal-Singer, R.; Woodcock, A.; Singh, D.; Goodacre, R.; Paul Thomas, C.L.; Fowler, S.J. Non-invasive metabolomic analysis of breath using differential mobility spectrometry in patients with chronic obstructive pulmonary disease and healthy smokers. Analyst 2010, 135, 315-320. [CrossRef] [PubMed]

36. Zhou, X.; Xue, Z.; Chen, X.; Huang, C.; Bai, W.; Lu, Z.; Wang, T. Nanomaterial-based gas sensors used for breath diagnosis. J. Mater. Chem. B 2020, 8, 3231-3248. [CrossRef] [PubMed]

37. Ratiu, I.A.; Ligor, T.; Bocos-Bintintan, V.; Mayhew, C.A.; Buszewski, B. Volatile Organic Compounds in Exhaled Breath as Fingerprints of Lung Cancer, Asthma and COPD. J. Clin. Med. 2020, 10, 32. [CrossRef] [PubMed]

38. Tisch, U.; Haick, H. Nanomaterials for cross-reactive sensor arrays. MRS Bull. 2010, 35, 797-803. [CrossRef]

39. Van Der Schee, M.P.; Fens, N.; Brinkman, P.; Bos, L.D.J.; Angelo, M.D.; Nijsen, T.M.E.; Raabe, R.; Knobel, H.H.; Vink, T.J.; Sterk, P.J. Effect of transportation and storage using sorbent tubes of exhaled breath samples on diagnostic accuracy of electronic nose analysis. J. Breath Res. 2013, 7, 016002. [CrossRef]

40. Behera, B.; Joshi, R.; Anil Vishnu, G.K.; Bhalerao, S.; Pandya, H.J. Electronic nose: A non-invasive technology for breath analysis of diabetes and lung cancer patients. J. Breath Res. 2019, 13, 24001. [CrossRef]

41. Madianos, L.; Skotadis, E.; Patsiouras, L.; Filippidou, M.K.; Chatzandroulis, S.; Tsoukalas, D. Nanoparticle based gas-sensing array for pesticide detection. J. Environ. Chem. Eng. 2018, 6, 6641-6646. [CrossRef]

42. Konvalina, G.; Haick, H. Effect of humidity on nanoparticle-based chemiresistors: A comparison between synthetic and real-world samples. ACS Appl. Mater. Interfaces 2012, 4, 317-325. [CrossRef]

43. Shehada, N.; Cancilla, J.C.; Torrecilla, J.S.; Pariente, E.S.; Brönstrup, G.; Christiansen, S.; Johnson, D.W.; Leja, M.; Davies, M.P.A.; Liran, O.; et al. Silicon Nanowire Sensors Enable Diagnosis of Patients via Exhaled Breath. ACS Nano 2016, 10, 7047-7057. [CrossRef]

44. Kahn, N.; Lavie, O.; Paz, M.; Segev, Y.; Haick, H. Dynamic Nanoparticle-Based Flexible Sensors: Diagnosis of Ovarian Carcinoma from Exhaled Breath. Nano Lett. 2015, 15, 7023-7028. [CrossRef]

45. Phillips, C.O.; Syed, Y.; Parthaláin, N.M.; Zwiggelaar, R.; Claypole, T.C.; Lewis, K.E. Machine learning methods on exhaled volatile organic compounds for distinguishing COPD patients from healthy controls. J. Breath Res. 2012, 6, 036003. [CrossRef] [PubMed]

46. Coyle, S.; Curto, V.F.; Benito-Lopez, F.; Florea, L.; Diamond, D. Wearable Bio and Chemical Sensors. In Wearable Sensors: Fundamentals, Implementation and Applications, 1st ed.; Sazonov, E., Neuman, M.R., Eds.; Elsevier: Amsterdam, The Netherlands, 2014; pp. 65-83.

47. Skotadis, E.; Tang, J.; Tsouti, V.; Tsoukalas, D. Chemiresistive sensor fabricated by the sequential ink-jet printing deposition of a gold nanoparticle and polymer layer. Microelectron. Eng. 2010, 87, 2258-2263. [CrossRef]

48. Skotadis, E.; Tanner, J.L.; Stathopoulos, S.; Tsouti, V.; Tsoukalas, D. Chemical sensing based on double layer PHEMA polymer and platinum nanoparticle films. Sens. Actuators B Chem. 2012, 175, 85-91. [CrossRef]

49. Kus, F.; Altinkok, C.; Zayim, E.; Erdemir, S.; Tasaltin, C.; Gurol, I. Surface acoustic wave (SAW) sensor for volatile organic compounds (VOCs) detection with calix[4]arene functionalized Gold nanorods (AuNRs) and silver nanocubes (AgNCs). Sens. Actuators B Chem. 2021, 330, 129402. [CrossRef]

50. Zhang, T.; Mubeen, S.; Myung, N.V.; Deshusses, M.A. Recent progress in carbon nanotube-based gas sensors. Nanotechnology 2008, 19, 332001. [CrossRef] 
51. Haick, H. Chemical sensors based on molecularly modified metallic nanoparticles. J. Phys. D. Appl. Phys. 2007, 40, 7173-7186. [CrossRef]

52. Viespe, C.; Miu, D. Characteristics of Surface Acoustic Wave Sensors with Nanoparticles Embedded in Polymer Sensitive Layers for VOC Detection. Sensors 2018, 18, 2401. [CrossRef]

53. Yoon, J.W.; Lee, J.H. Toward breath analysis on a chip for disease diagnosis using semiconductor-based chemiresistors: Recent progress and future perspectives. Lab. Chip 2017, 17, 3537-3557. [CrossRef]

54. Vasilescu, A.; Hrinczenko, B.; Swain, G.M.; Peteu, S.F. Exhaled breath biomarker sensing. Biosens. Bioelectron. 2021, 182, 113193. [CrossRef]

55. Moon, H.G.; Choi, Y.R.; Shim, Y.S.; Choi, K.I.; Lee, J.H.; Kim, J.S.; Yoon, S.J.; Park, H.H.; Kang, C.Y.; Jang, H.W. Extremely sensitive and selective $\mathrm{NO}$ probe based on villi-like $\mathrm{WO}_{3}$ nanostructures for application to exhaled breath analyzers. ACS Appl. Mater. Interfaces 2013, 5, 10591-10596. [CrossRef]

56. Koo, W.T.; Choi, S.J.; Kim, N.H.; Jang, J.S.; Kim, I.D. Catalyst-decorated hollow $\mathrm{WO}_{3}$ nanotubes using layer-by-layer self-assembly on polymeric nanofiber templates and their application in exhaled breath sensor. Sens. Actuators B Chem. 2016, 223, 301-310. [CrossRef]

57. Choi, S.J.; Fuchs, F.; Demadrille, R.; Grévin, B.; Jang, B.H.; Lee, S.J.; Lee, J.H.; Tuller, H.L.; Kim, I.D. Fast responding exhaled-breath sensors using $\mathrm{WO}_{3}$ hemitubes functionalized by graphene-based electronic sensitizers for diagnosis of diseases. ACS Appl. Mater. Interfaces 2014, 6, 9061-9070. [CrossRef] [PubMed]

58. Shin, J.; Choi, S.J.; Lee, I.; Youn, D.Y.; Park, C.O.; Lee, J.H.; Tuller, H.L.; Kim, I.D. Thin-wall assembled SnO ${ }_{2}$ fibers functionalized by catalytic Pt nanoparticles and their superior exhaled-breath-sensing properties for the diagnosis of diabetes. Adv. Funct. Mater. 2013, 23, 2357-2367. [CrossRef]

59. Masuda, Y.; Itoh, T.; Shin, W.; Kato, $\mathrm{K} . \mathrm{SnO}_{2}$ nanosheet/nanoparticle detector for the sensing of 1-nonanal gas produced by lung cancer. Sci. Rep. 2015, 5, 10122. [CrossRef] [PubMed]

60. Giebelhaus, I.; Varechkina, E.; Fischer, T.; Rumyantseva, M.; Ivanov, V.; Gaskov, A.; Morante, J.R.; Arbiol, J.; Tyrra, W.; Mathur, S. One-dimensional CuO-SnO 2 p-n heterojunctions for enhanced detection of $\mathrm{H}_{2} \mathrm{~S}$. J. Mater. Chem. A 2013, 1, 11261-11268. [CrossRef]

61. Wei, C.; Dai, L.; Roy, A.; Tolle, T.B. Multifunctional chemical vapor sensors of aligned carbon nanotube and polymer composites. J. Am. Chem. Soc. 2006, 128, 1412-1413. [CrossRef]

62. Ionescu, R.; Broza, Y.; Shaltieli, H.; Sadeh, D.; Zilberman, Y.; Feng, X.; Glass-Marmor, L.; Lejbkowicz, I.; Müllen, K.; Miller, A.; et al. Detection of multiple sclerosis from exhaled breath using bilayers of polycyclic aromatic hydrocarbons and single-wall carbon nanotubes. ACS Chem. Neurosci. 2011, 2, 687-693. [CrossRef] [PubMed]

63. Akbari-Saatlu, M.; Procek, M.; Mattsson, C.; Thungström, G.; Nilsson, H.E.; Xiong, W.; Xu, B.; Li, Y.; Radamson, H.H. Silicon nanowires for gas sensing: A review. Nanomaterials 2020, 10, 2215. [CrossRef]

64. Wang, B.; Haick, H. Effect of chain length on the sensing of volatile organic compounds by means of silicon nanowires. ACS Appl. Mater. Interfaces 2013, 5, 5748-5756. [CrossRef]

65. Ermanok, R.; Assad, O.; Zigelboim, K.; Wang, B.; Haick, H. Discriminative power of chemically sensitive silicon nanowire field effect transistors to volatile organic compounds. ACS Appl. Mater. Interfaces 2013, 5, 11172-11183. [CrossRef]

66. Wang, B.; Cancilla, J.C.; Torrecilla, J.S.; Haick, H. Artificial sensing intelligence with silicon nanowires for ultraselective detection in the gas phase. Nano Lett. 2014, 14, 933-938. [CrossRef]

67. Choi, S.J.; Kim, I.D. Recent Developments in 2D Nanomaterials for Chemiresistive-Type Gas Sensors. Electron. Mater. Lett. 2018, 14, 221-260. [CrossRef]

68. Jeong, S.; Kim, J.; Lee, J. Rational Design of Semiconductor-Based Chemiresistors and their Libraries for Next-Generation Artificial Olfaction. Adv. Mater. 2020, 32, 2002075. [CrossRef] [PubMed]

69. Chen, W.Y.; Yen, C.C.; Xue, S.; Wang, H.; Stanciu, L.A. Surface Functionalization of Layered Molybdenum Disulfide for the Selective Detection of Volatile Organic Compounds at Room Temperature. ACS Appl. Mater. Interfaces 2019, 11, 34135-34143. [CrossRef]

70. $\mathrm{Chu}, \mathrm{X} . ; \mathrm{Hu}, \mathrm{R} . ;$ Wang, J.; Dong, Y.; Zhang, W.; Bai, L.; Sun, W. Preparation and gas sensing properties of graphene-Zn $\mathrm{Z}_{2} \mathrm{SnO}_{4}$ composite materials. Sens. Actuators B Chem. 2017, 251, 120-126. [CrossRef]

71. Tang, J.; Skotadis, E.; Stathopoulos, S.; Roussi, V.; Tsouti, V.; Tsoukalas, D. PHEMA functionalization of gold nanoparticles for vapor sensing: Chemi-resistance, chemi-capacitance and chemi-impedance. Sens. Actuators B Chem. 2012, 170, 129-136. [CrossRef]

72. Di Natale, C.; Paolesse, R.; Martinelli, E.; Capuano, R. Solid-state gas sensors for breath analysis: A review. Anal. Chim. Acta 2014, 824, 1-17. [CrossRef] [PubMed]

73. Liu, C.; Wyszynski, B.; Yatabe, R.; Hayashi, K.; Toko, K. Molecularly imprinted sol-gel-based QCM sensor arrays for the detection and recognition of volatile aldehydes. Sensors 2017, 17, 382. [CrossRef]

74. Chen, W.; Wang, Z.; Gu, S.; Wang, J. Chemical Detection of hexanal in humid circumstances using hydrophobic molecularly imprinted polymers composite. Sens. Actuators B. Chem. 2019, 291, 141-147. [CrossRef]

75. Amor, R.E.; Nakhleh, M.K.; Barash, O.; Haick, H. Breath analysis of cancer in the present and the future. Eur. Respir. Rev. 2019, 28, 190002. [CrossRef]

76. Güntner, A.T.; Koren, V.; Chikkadi, K.; Righettoni, M.; Pratsinis, S.E. E-Nose Sensing of Low-ppb Formaldehyde in Gas Mixtures at High Relative Humidity for Breath Screening of Lung Cancer? ACS Sens. 2016, 1, 528-535. [CrossRef] 
77. Kumar, D.; Chaturvedi, P.; Saho, P.; Jha, P.; Chouksey, A.; Lal, M.; Rawat, J.S.B.S.; Tandon, R.P.; Chaudhury, P.K. Effect of single wall carbon nanotube networks on gas sensor response and detection limit. Sens. Actuators B Chem. 2017, 240, 1134-1140. [CrossRef]

78. Young, S.J.; Lin, Z.D. Ethanol gas sensors based on multi-wall carbon nanotubes on oxidized Si substrate. Microsyst. Technol. 2018, 24, 55-58. [CrossRef]

79. Hanh, N.H.; van Duy, L.; Hung, C.M.; Xuan, C.T.; van Duy, N.; Hoa, N.D. High-performance acetone gas sensor based on $\mathrm{Pt}-\mathrm{Zn}_{2} \mathrm{SnO}_{4}$ hollow octahedra for diabetic diagnosis. J. Alloys Compd. 2021, 886, 161284. [CrossRef]

80. Panes-Ruiz, L.A.; Riemenschneider, L.; Al Chawa, M.M.; Löffler, M.; Rellinghaus, B.; Tetzlaff, R.; Bezugly, V.; Ibarlucea, B.; Cuniberti, G. Selective and self-validating breath-level detection of hydrogen sulfide in humid air by gold nanoparticlefunctionalized nanotube arrays. Nano Res. 2021. [CrossRef]

81. Choi, S.J.; Jang, B.H.; Lee, S.J.; Min, B.K.; Rothschild, A.; Kim, I.D. Selective detection of acetone and hydrogen sulfide for the diagnosis of diabetes and halitosis using $\mathrm{SnO}_{2}$ nanofibers functionalized with reduced graphene oxide nanosheets. ACS Appl. Mater. Interfaces 2014, 6, 2588-2597. [CrossRef] [PubMed]

82. Zhang, L.; Li, C.; Liu, A.; Shi, G. Electrosynthesis of graphene oxide/polypyrene composite films and their applications for sensing organic vapors. J. Mater. Chem. 2012, 22, 8438-8443. [CrossRef]

83. Lee, J.S.; Jeong, D.W.; Byun, Y.T. Porphyrin nanofiber/single-walled carbon nanotube nanocomposite-based sensors for monitoring hydrogen peroxide vapor. Sens. Actuators B Chem. 2020, 306, 127518. [CrossRef]

84. Bachhav, S.G.; Patil, D.R. Study of Polypyrrole-Coated MWCNT Nanocomposites for Ammonia Sensing at Room Temperature. J. Mater. Sci. Chem. Eng. 2015, 3, 30-44. [CrossRef]

85. Abdulla, S.; Mathew, T.L.; Pullithadathil, B. Highly sensitive, room temperature gas sensor based on polyaniline-multiwalled carbon nanotubes (PANI/MWCNTs) nanocomposite for trace-level ammonia detection. Sens. Actuators B Chem. 2015, 221, 1523-1534. [CrossRef]

86. Binson, V.A.; Subramoniam, M. Design and development of an e-nose system for the diagnosis of pulmonary diseases. Acta Bioeng. Biomech. 2021, 23, 35-44. [CrossRef] [PubMed]

87. Binson, V.A.; Subramoniam, M.; Mathew, L. Discrimination of COPD and lung cancer from controls through breath analysis using a self-developed e-nose. J. Breath Res. 2021, 15, 46003. [CrossRef]

88. Marzorati, D.; Mainardi, L.; Sedda, G.; Gasparri, R.; Spaggiari, L.; Cerveri, P. MOS sensors array for the discrimination of lung cancer and at-risk subjects with exhaled breath analysis. Chemosensors 2021, 9, 209. [CrossRef]

89. Chang, J.; Lee, D.; Ban, S.; Oh, J.; Youn, M.; Kim, S.; Park, S.; Persaud, K.; Jheon, S. Chemical Analysis of volatile organic compounds in exhaled breath for lung cancer diagnosis using a sensor system. Sens. Actuators B. Chem. 2018, 255, 800-807. [CrossRef]

90. Brinkman, P.; van de Pol, M.A.; Gerritsen, M.G.; Bos, L.D.; Dekker, T.; Smids, B.S.; Sinha, A.; Majoor, C.J.; Sneeboer, M.M.; Knobel, H.H.; et al. Exhaled breath profiles in the monitoring of loss of control and clinical recovery in asthma. Clin. Exp. Allergy 2017, 47, 1159-1169. [CrossRef] [PubMed]

91. De Vries, R.; Brinkman, P.; Van Der Schee, M.P.; Fens, N.; Dijkers, E.; Bootsma, S.K.; De Jongh, F.H.C.; Sterk, P.J. Integration of electronic nose technology with spirometry: Validation of a new approach for exhaled breath analysis. J. Breath Res. 2015, 9, 046001. [CrossRef]

92. Krauss, E.; Haberer, J.; Barreto, G.; Degen, M.; Seeger, W.; Guenther, A. Recognition of breathprints of lung cancer and chronic obstructive pulmonary disease using the Aeonose ${ }^{\circledR}$ electronic nose. J. Breath Res. 2020, 14, 046004. [CrossRef]

93. Wang, X.R.; Lizier, J.T.; Berna, A.Z.; Bravo, F.G.; Trowell, S.C. Human breath-print identification by E-nose, using informationtheoretic feature selection prior to classification. Sens. Actuators B Chem. 2015, 217, 165-174. [CrossRef]

94. Kaushal, P.; Mudhalwadkar, R.P. Pellet sensor based asthma detection system using exhaled breath analysis. In Proceedings of the 2015 International Conference on Industrial Instrumentation and Control (ICIC), Pune, India, 28-30 May 2015; pp. 139-142. [CrossRef]

95. Xu, Y.; Lee, H.; Hu, Y.; Huang, J.; Kim, S.; Yun, M. Detection and identification of breast cancer volatile organic compounds biomarkers using highly-sensitive single nanowire array on a chip. J. Biomed. Nanotechnol. 2013, 9, 1164-1172. [CrossRef]

96. Choi, S.J.; Ku, K.H.; Kim, B.J.; Kim, I.D. Novel Templating Route Using Pt Infiltrated Block Copolymer Microparticles for Catalytic Pt Functionalized Macroporous $\mathrm{WO}_{3}$ Nanofibers and Its Application in Breath Pattern Recognition. ACS Sens. 2016, 1, $1124-1131$. [CrossRef]

97. Hong, D.; Hoon, T.; Sohn, W.; Min, J.; Shim, Y. Chemical Au decoration of vertical hematite nanotube arrays for further selective detection of acetone in exhaled breath. Sens. Actuators B. Chem. 2018, 274, 587-594. [CrossRef]

98. Choi, S.J.; Lee, I.; Jang, B.H.; Youn, D.Y.; Ryu, W.H.; Park, C.O.; Kim, I.D. Selective diagnosis of diabetes using Pt-functionalized $\mathrm{WO}_{3}$ hemitube networks as a sensing layer of acetone in exhaled breath. Anal. Chem. 2013, 85, 1792-1796. [CrossRef] [PubMed]

99. Kim, G.S.; Park, Y.; Shin, J.; Song, Y.G.; Kang, C.Y. Metal oxide nanorods-based sensor array for selective detection of biomarker gases. Sensors 2021, 21, 1922. [CrossRef] [PubMed]

100. Moon, H.G.; Jung, Y.; Han, S.D.; Shim, Y.S.; Shin, B.; Lee, T.; Kim, J.S.; Lee, S.; Jun, S.C.; Park, H.H.; et al. Chemiresistive Electronic Nose toward Detection of Biomarkers in Exhaled Breath. ACS Appl. Mater. Interfaces 2016, 8, 20969-20976. [CrossRef] [PubMed]

101. Carlos, S.; Santos, P.; Sayago, I. Graphene-Doped Tin Oxide Nanofibers and Nanoribbons as Gas Sensors to Detect Biomarkers of Different Diseases through the Breath. Sensors 2020, 20, 7223. [CrossRef] 
102. Liu, B.; Huang, Y.; Kam, K.W.; Cheung, W.F.; Zhao, N.; Zheng, B. Functionalized graphene-based chemiresistive electronic nose for discrimination of disease-related volatile organic compounds. Biosens. Bioelectron. X 2019, 1, 100016. [CrossRef]

103. Sarkar, T.; Srinives, S.; Rodriquez, A.; Mulchandani, A. Single-walled Carbon Nanotube-Calixarene Based Chemiresistor for Volatile Organic Compounds. Electroanalysis 2018, 30, 2077-2084. [CrossRef]

104. Abdelhalim, A.; Winkler, M.; Loghin, F.; Zeiser, C.; Lugli, P.; Abdellah, A. Highly sensitive and selective carbon nanotube-based gas sensor arrays functionalized with different metallic nanoparticles. Sens. Actuators B Chem. 2015, 220, 1288-1296. [CrossRef]

105. Gao, Z.; Lou, Z.; Chen, S.; Li, L.; Jiang, K.; Fu, Z.; Han, W.; Shen, G. Fiber gas sensor-integrated smart face mask for roomtemperature distinguishing of target gases. Nano Res. 2018, 11, 511-519. [CrossRef]

106. Gautam, V.; Kumar, A.; Kumar, R.; Jain, V.K.; Nagpal, S. Silicon nanowires/reduced graphene oxide nanocomposite based novel sensor platform for detection of cyclohexane and formaldehyde. Mater. Sci. Semicond. Process. 2021, 123, 105571. [CrossRef]

107. Freddi, S.; Emelianov, A.V.; Bobrinetskiy, I.I.; Drera, G.; Pagliara, S.; Kopylova, D.S.; Chiesa, M.; Santini, G.; Mores, N.; Moscato, U.; et al. Development of a Sensing Array for Human Breath Analysis Based on SWCNT Layers Functionalized with Semiconductor Organic Molecules. Adv. Healthc. Mater. 2020, 9, 2000377. [CrossRef] [PubMed]

108. Tan, J.L.; Yong, Z.X.; Liam, C.K. Using a chemiresistor-based alkane sensor to distinguish exhaled breaths of lung cancer patients from subjects with no lung cancer. J. Thorac. Dis. 2016, 8, 2772-2783. [CrossRef] [PubMed]

109. Welearegay, T.G.; Diouani, M.F.; Österlund, L.; Ionescu, F.; Belgacem, K.; Smadhi, H.; Khaled, S.; Kidar, A.; Cindemir, U.; Laouini, D.; et al. Ligand-Capped Ultrapure Metal Nanoparticle Sensors for the Detection of Cutaneous Leishmaniasis Disease in Exhaled Breath. ACS Sens. 2018, 3, 2532-2540. [CrossRef] [PubMed]

110. Castro, M.; Kumar, B.; Feller, J.F.; Haddi, Z.; Amari, A.; Bouchikhi, B. Chemical Novel e-nose for the discrimination of volatile organic biomarkers with an array of carbon nanotubes (CNT) conductive polymer nanocomposites (CPC) sensors. Sens. Actuators B. Chem. 2011, 159, 213-219. [CrossRef]

111. Karban, A.; Nakhleh, M.K.; Cancilla, J.C.; Vishinkin, R.; Rainis, T.; Koifman, E.; Jeries, R.; Ivgi, H.; Torrecilla, J.S.; Haick, H. Programmed Nanoparticles for Tailoring the Detection of Inflammatory Bowel Diseases and Irritable Bowel Syndrome Disease via Breathprint. Adv. Healthc. Mater. 2016, 5, 2339-2344. [CrossRef] [PubMed]

112. Broza, Y.Y.; Khatib, S.; Gharra, A.; Krilaviciute, A.; Amal, H.; Polaka, I.; Parshutin, S.; Kikuste, I.; Gasenko, E.; Skapars, R.; et al. Screening for gastric cancer using exhaled breath samples. Br. J. Surg. 2019, 106, 1122-1125. [CrossRef]

113. Marom, O.; Nakhoul, F.; Tisch, U.; Shiban, A.; Abassi, Z.; Haick, H. Gold nanoparticle sensors for detecting chronic kidney disease and disease progression. Nanomedicine 2012, 7, 639-650. [CrossRef]

114. Tisch, U.; Schlesinger, I.; Ionescu, R.; Nassar, M.; Axelrod, N.; Robertman, D.; Tessler, Y.; Azar, F.; Marmur, A.; AharonPeretz, J.; et al. Detection of Alzheimer's and Parkinson's disease from exhaled breath using nanomaterial-based sensors. Nanomedicine 2013, 8, 43-56. [CrossRef]

115. Nakhleh, M.K.; Amal, H.; Jeries, R.; Broza, Y.Y.; Aboud, M.; Gharra, A.; Ivgi, H.; Khatib, S.; Badarneh, S.; Har-Shai, L.; et al. Diagnosis and Classification of 17 Diseases from 1404 Subjects via Pattern Analysis of Exhaled Molecules. ACS Nano 2017, 11, 112-125. [CrossRef]

116. Shuster, G.; Gallimidi, Z.; Reiss, A.H.; Dovgolevsky, E.; Billan, S.; Abdah-Bortnyak, R.; Kuten, A.; Engel, A.; Shiban, A.; Tisch, U.; et al. Classification of breast cancer precursors through exhaled breath. Breast Cancer Res. Treat. 2011, 126, 791-796. [CrossRef]

117. Licht, J.C.; Grasemann, H. Potential of the electronic nose for the detection of respiratory diseases with and without infection. Int. J. Mol. Sci. 2020, 21, 9416. [CrossRef]

118. Bikov, A.; Lázár, Z.; Horvath, I. Established methodological issues in electronic nose research: How far are we from using these instruments in clinical settings of breath analysis? J. Breath Res. 2015, 9, 034001. [CrossRef]

119. Tenero, L.; Sandri, M.; Piazza, M.; Paiola, G.; Zaffanello, M.; Piacentini, G. Electronic nose in discrimination of children with uncontrolled asthma. J. Breath Res. 2020, 14, 046003. [CrossRef] [PubMed]

120. Fens, N.; De Nijs, S.B.; Peters, S.; Dekker, T.; Knobel, H.H.; Vink, T.J.; Willard, N.P.; Zwinderman, A.H.; Krouwels, F.H.; Lutter, R.; et al. Exhaled air molecular profiling in relation to inflammatory subtype and activity in COPD. Eur. Respir. J. 2011, 38, 1301-1309. [CrossRef] [PubMed]

121. De León-Martínez, L.D.; Rodríguez-Aguilar, M.; Gorocica-Rosete, P.; Domínguez-Reyes, C.A.; Martínez-Bustos, V.; TenorioTorres, J.A.; Ornelas-Rebolledo, O.; Cruz-Ramos, J.A.; Balderas-Segura, B.; Flores-Ramírez, R. Identification of profiles of volatile organic compounds in exhaled breath by means of an electronic nose as a proposal for a screening method for breast cancer: A case-control study. J. Breath Res. 2020, 14, 046009. [CrossRef]

122. Chen, C.Y.; Lin, W.C.; Yang, H.Y. Diagnosis of ventilator-associated pneumonia using electronic nose sensor array signals: Solutions to improve the application of machine learning in respiratory research. Respir. Res. 2020, 21, 45. [CrossRef]

123. Skotadis, E.; Mousadakos, D.; Katsabrokou, K.; Stathopoulos, S.; Tsoukalas, D. Flexible polyimide chemical sensors using platinum nanoparticles. Sens. Actuators B Chem. 2013, 189, 106-112. [CrossRef]

124. Skotadis, E.; Kanaris, A.; Aslanidis, E.; Kalatzis, N.; Chatzipapadopoulos, F.; Marianos, N.; Tsoukalas, D. Identification of two commercial pesticides by a nanoparticle gas-sensing array. Sensors 2021, 21, 5803. [CrossRef] [PubMed]

125. Shehada, N.; Brönstrup, G.; Funka, K.; Christiansen, S.; Leja, M.; Haick, H. Ultrasensitive silicon nanowire for real-world gas sensing: Noninvasive diagnosis of cancer from breath volatolome. Nano Lett. 2015, 15, 1288-1295. [CrossRef] [PubMed] 
126. Obermeier, J.; Trefz, P.; Wex, K.; Sabel, B.; Schubert, J.K.; Miekisch, W. Electrochemical sensor system for breath analysis of aldehydes, CO and NO. J. Breath Res. 2015, 9, 016008. [CrossRef]

127. Mazzone, P.J.; Wang, X.; Xu, Y. Exhaled Breath Analysis with a Colorimetric Sensor Array for the Identification and Characterization of Lung Cancer. J. Thorac. Oncol. 2012, 7, 137-142. [CrossRef]

128. Silva, L.I.B.; Freitas, A.C.; Rocha-Santos, T.A.P.; Pereira, M.E.; Duarte, A.C. Breath analysis by optical fiber sensor for the determination of exhaled organic compounds with a view to diagnostics. Talanta 2011, 83, 1586-1594. [CrossRef]

129. Chen, X.; Cao, M.; Li, Y.; Hu, W.; Wang, P.; Ying, K.; Pan, H. A study of an electronic nose for detection of lung cancer based on a virtual SAW gas sensors array and imaging recognition method. Meas. Sci. Technol. 2005, 16, 1535-1546. [CrossRef]

130. Incalzi, R.A.; Pennazza, G.; Scarlata, S.; Santonico, M.; Petriaggi, M.; Chiurco, D.; Pedone, C.; D'Amico, A. Reproducibility and Respiratory Function Correlates of Exhaled Breath Fingerprint in Chronic Obstructive Pulmonary Disease. PLoS ONE 2012, 7, e45396. [CrossRef] [PubMed]

131. Montuschi, P.; Santonico, M.; Mondino, C. Diagnostic Performance of an Electronic Nose, Fractional Exhaled Nitric Oxide, and Lung Function Testing in Asthma. Chest 2010, 137, 790-796. [CrossRef]

132. Santonico, M.; Lucantoni, G.; Pennazza, G.; Capuano, R.; Galluccio, G.; Roscioni, C.; La Delfa, G.; Consoli, D.; Martinelli, E.; Paolesse, R.; et al. In situ detection of lung cancer volatile fingerprints using bronchoscopic air-sampling. Lung Cancer 2012, 77, 46-50. [CrossRef]

133. Gasparri, R.; Santonico, M.; Valentini, C.; Sedda, G.; Borri, A.; Petrella, F.; Maisonneuve, P.; Pennazza, G.; D'Amico, A.; Di Natale, C.; et al. Volatile signature for the early diagnosis of lung cancer. J. Breath Res. 2016, 10, 016007. [CrossRef] [PubMed]

134. Zetola, N.M.; Modongo, C.; Matsiri, O.; Tamuhla, T.; Mbongwe, B.; Matlhagela, K.; Sepako, E.; Catini, A.; Sirugo, G.; Martinelli, E.; et al. Diagnosis of pulmonary tuberculosis and assessment of treatment response through analyses of volatile compound patterns in exhaled breath samples. J. Infect. 2017, 74, 367-376. [CrossRef] [PubMed]

135. Pennazza, G.; Marchetti, E.; Santonico, M.; Mantini, G.; Mummolo, S.; Marzo, G.; Paolesse, R.; D’Amico, A.; Di Natale, C. Application of a quartz microbalance based gas sensor array for the study of halitosis. J. Breath Res. 2008, 2, 017009. [CrossRef]

136. Fasola, S.; Ferrante, G.; Sabatini, A.; Santonico, M.; Zompanti, A.; Grasso, S.; Antonelli Incalzi, R.; La Grutta, S. Repeatability of exhaled breath fingerprint collected by a modern sampling system in asthmatic and healthy children. J. Breath Res. 2019, 13, 036007. [CrossRef]

137. Zhang, Y.; Gao, G.; Liu, H.; Fu, H.; Fan, J.; Wang, K.; Chen, Y.; Li, B.; Zhang, C.; Zhi, X.; et al. Identification of volatile biomarkers of gastric cancer cells and ultrasensitive electrochemical detection based on sensing interface of Au-Ag alloy coated MWCNTs. Theranostics 2014, 4, 154-162. [CrossRef]

138. Bahos, F.A.; Sainz-Vidal, A.; Sánchez-Pérez, C.; Saniger, J.M.; Gràcia, I.; Saniger-Alba, M.M.; Matatagui, D. ZIF nanocrystal-based surface acousticwave (SAW) electronic nose to detect diabetes in human breath. Biosensors 2019, 9, 4. [CrossRef]

139. Tao, W.; Lin, P.; Ai, Y.; Wang, H.; Ke, S.; Zeng, X. Multichannel quartz crystal microbalance array: Fabrication, evaluation, application in biomarker detection. Anal. Biochem. 2016, 494, 85-92. [CrossRef]

140. Zhao, S.; Lei, J.; Huo, D.; Hou, C.; Luo, X.; Wu, H.; Fa, H.; Yang, M. Chemical A colorimetric detector for lung cancer related volatile organic compounds based on cross-response mechanism. Sens. Actuators B. Chem. 2018, 256, 543-552. [CrossRef]

141. Huo, D.; Xu, Y.; Hou, C.; Yang, M.; Fa, H. A novel optical chemical sensor based AuNR-MTPP and dyes for lung cancer biomarkers in exhaled breath identification. Sens. Actuators B Chem. 2014, 199, 446-456. [CrossRef]

142. Feng, P.; Shao, F.; Shi, Y.; Wan, Q. Gas sensors based on semiconducting nanowire field-effect transistors. Sensors 2014, 14, 17406. [CrossRef]

143. Fraden, J. Chemical sensors. In Handbook of Modern Sensors: Physics, Designs, and Applications, 4th ed.; Springer: New York, NY, USA, 2010; pp. 569-606.

144. Paska, Y.; Stelzner, T.; Assad, O.; Tisch, U.; Christiansen, S.; Haick, H. Molecular gating of silicon nanowire field-effect transistors with nonpolar analytes. ACS Nano 2012, 6, 335-345. [CrossRef]

145. Chen, G.; Paronyan, T.M.; Pigos, E.M.; Harutyunyan, A.R. Enhanced gas sensing in pristine carbon nanotubes under continuous ultraviolet light illumination. Sci. Rep. 2012, 2, 343. [CrossRef]

146. Zhou, C.; Zhao, J.; Ye, J.; Tange, M.; Zhang, X.; Xu, W.; Zhang, K.; Okazaki, T.; Cui, Z. Printed thin-film transistors and NO 2 gas sensors based on sorted semiconducting carbon nanotubes by isoindigo-based copolymer. Carbon N. Y. 2016, 108, 372-380. [CrossRef]

147. Chang, Y.W.; Oh, J.S.; Yoo, S.H.; Choi, H.H.; Yoo, K.H. Electrically refreshable carbon-nanotube-based gas sensors. Nanotechnology 2007, 18, 435504. [CrossRef]

148. Huo, N.; Yang, S.; Wei, Z.; Li, S.S.; Xia, J.B.; Li, J. Photoresponsive and Gas Sensing Field-Effect Transistors based on Multilayer $\mathrm{WS}_{2}$ Nanoflakes. Sci. Rep. 2014, 4, 5209. [CrossRef]

149. Li, H.; Yin, Z.; He, Q.; Li, H.; Huang, X.; Lu, G.; Fam, D.W.H.; Tok, A.I.Y.; Zhang, Q.; Zhang, H. Fabrication of single- and multilayer $\mathrm{MoS}_{2}$ film-based field-effect transistors for sensing $\mathrm{NO}$ at room temperature. Small 2012, 8, 63-67. [CrossRef] [PubMed]

150. Late, D.J.; Huang, Y.K.; Liu, B.; Acharya, J.; Shirodkar, S.N.; Luo, J.; Yan, A.; Charles, D.; Waghmare, U.V.; Dravid, V.P.; et al Sensing behavior of atomically thin-layered $\mathrm{MoS}_{2}$ transistors. ACS Nano 2013, 7, 4879-4891. [CrossRef] [PubMed]

151. Chen, B.; Liu, H.; Li, X.; Lu, C.; Ding, Y.; Lu, B. Fabrication of a graphene field effect transistor array on microchannels for ethanol sensing. Appl. Surf. Sci. 2012, 258, 1971-1975. [CrossRef] 
152. Cui, S.; Pu, H.; Mattson, E.C.; Wen, Z.; Chang, J.; Hou, Y.; Hirschmugl, C.J.; Chen, J. Ultrasensitive chemical sensing through facile tuning defects and functional groups in reduced graphene oxide. Anal. Chem. 2014, 86, 7516-7522. [CrossRef]

153. Mortazavi Zanjani, S.M.; Sadeghi, M.M.; Holt, M.; Chowdhury, S.F.; Tao, L.; Akinwande, D. Enhanced sensitivity of graphene ammonia gas sensors using molecular doping. Appl. Phys. Lett. 2016, 108, 033106. [CrossRef]

154. Cho, B.; Yoon, J.; Lim, S.K.; Kim, A.R.; Kim, D.H.; Park, S.G.; Kwon, J.D.; Lee, Y.J.; Lee, K.H.; Lee, B.H.; et al. Chemical Sensing of 2D Graphene/ $\mathrm{MoS}_{2}$ Heterostructure device. ACS Appl. Mater. Interfaces 2015, 7, 16775-16780. [CrossRef]

155. Van Hieu, N.; Dung, N.Q.; Tam, P.D.; Trung, T.; Chien, N.D. Thin film polypyrrole/SWCNTs nanocomposites-based NH N $_{3}$ sensor operated at room temperature. Sens. Actuators B Chem. 2009, 140, 500-507. [CrossRef]

156. Mubeen, S.; Lim, J.H.; Srirangarajan, A.; Mulchandani, A.; Deshusses, M.A.; Myung, N.V. Gas sensing mechanism of gold nanoparticles decorated single-walled carbon nanotubes. Electroanalysis 2011, 23, 2687-2692. [CrossRef]

157. Kaiser, A.; Ceja, E.T.; Liu, Y.; Huber, F.; Müller, R.; Herr, U.; Thonke, $\mathrm{K} \mathrm{H}_{2} \mathrm{~S}$ sensing for breath analysis with Au functionalized ZnO nanowires. Nanotechnology 2021, 32, 205505. [CrossRef]

158. Niskanen, A.O.; Colli, A.; White, R.; Li, H.W.; Spigone, E.; Kivioja, J.M. Silicon nanowire arrays as learning chemical vapour classifiers. Nanotechnology 2011, 22, 295502. [CrossRef] [PubMed]

159. Dattoli, E.N.; Davydov, A.V.; Benkstein, K.D. Tin oxide nanowire sensor with integrated temperature and gate control for multi-gas recognition. Nanoscale 2012, 4, 1760-1769. [CrossRef]

160. Li, D.; Hu, J.; Wu, R.; Lu, J.G. Conductometric chemical sensor based on individual CuO nanowires. Nanotechnology 2010, 21, 485502. [CrossRef]

161. Mahapatra, N.; Ben-Cohen, A.; Vaknin, Y.; Henning, A.; Hayon, J.; Shimanovich, K.; Greenspan, H.; Rosenwaks, Y. Electrostatic Selectivity of Volatile Organic Compounds Using Electrostatically Formed Nanowire Sensor. ACS Sens. $2018,3,709-715$. [CrossRef]

162. Chen, P.C.; Ishikawa, F.N.; Chang, H.K.; Ryu, K.; Zhou, C. A nanoelectronic nose: A hybrid nanowire/carbon nanotube sensor array with integrated micromachined hotplates for sensitive gas discrimination. Nanotechnology 2009, 20, 125503. [CrossRef] [PubMed]

163. Peng, G.; Tisch, U.; Haick, H. Detection of nonpolar molecules by means of carrier scattering in random networks of carbon nanotubes: Toward diagnosis of diseases via breath samples. Nano Lett. 2009, 9, 1362-1368. [CrossRef] [PubMed]

164. Maier, D.; Laubender, E.; Basavanna, A.; Schumann, S.; Güder, F.; Urban, G.A.; Dincer, C. Toward Continuous Monitoring of Breath Biochemistry: A Paper-Based Wearable Sensor for Real-Time Hydrogen Peroxide Measurement in Simulated Breath. ACS Sens. 2019, 4, 2945-2951. [CrossRef]

165. Chuang, M.Y.; Chen, C.C.; Zan, H.W.; Meng, H.F.; Lu, C.J. Organic Gas Sensor with an Improved Lifetime for Detecting Breath Ammonia in Hemodialysis Patients. ACS Sens. 2017, 2, 1788-1795. [CrossRef]

166. Zhang, J.; Jiang, G.; Goledzinowski, M.; Comeau, F.J.E.; Li, K.; Cumberland, T.; Lenos, J.; Xu, P.; Li, M.; Yu, A.; et al. Green Solid Electrolyte with Cofunctionalized Nanocellulose/Graphene Oxide Interpenetrating Network for Electrochemical Gas Sensors. Small Methods 2017, 1, 1700237. [CrossRef]

167. Queralto, N.; Berliner, A.N.; Goldsmith, B.; Martino, R.; Rhodes, P.; Lim, S.H. Detecting cancer by breath volatile organic compound analysis: A review of array-based sensors. J. Breath Res. 2014, 8, 027112. [CrossRef]

168. Wang, X.; Zhang, J.; Zhu, Z. Ammonia sensing characteristics of ZnO nanowires studied by quartz crystal microbalance. Appl. Surf. Sci. 2006, 252, 2404-2411. [CrossRef]

169. Van Quy, N.; Minh, V.A.; Van Luan, N.; Hung, V.N.; Van Hieu, N. Gas sensing properties at room temperature of a quartz crystal microbalance coated with $\mathrm{ZnO}$ nanorods. Sens. Actuators B Chem. 2011, 153, 188-193. [CrossRef]

170. Ding, B.; Kim, J.; Miyazaki, Y.; Shiratori, S. Electrospun nanofibrous membranes coated quartz crystal microbalance as gas sensor for $\mathrm{NH}_{3}$ detection. Sens. Actuators B Chem. 2004, 101, 373-380. [CrossRef]

171. Wang, X.; Ding, B.; Sun, M.; Yu, J.; Sun, G. Nanofibrous polyethyleneimine membranes as sensitive coatings for quartz crystal microbalance-based formaldehyde sensors. Sens. Actuators B Chem. 2010, 144, 11-17. [CrossRef]

172. Lukman Hekiem, N.L.; Md Ralib, A.A.; bt Mat Hattar, M.A.; Ahmad, F.B.; Nordin, A.N.; Rahim, R.A.; Za'bah, N.F. Advanced vapour sensing materials: Existing and latent to acoustic wave sensors for VOCs detection as the potential exhaled breath biomarkers for lung cancer. Sens. Actuators A Phys. 2021, 329, 112792. [CrossRef]

173. Si, P.; Mortensen, J.; Komolov, A.; Denborg, J.; Møller, P.J. Polymer coated quartz crystal microbalance sensors for detection of volatile organic compounds in gas mixtures. Anal. Chim. Acta 2007, 597, 223-230. [CrossRef]

174. Koshets, I.A.; Kazantseva, Z.I.; Shirshov, Y.M.; Cherenok, S.A.; Kalchenko, V.I. Calixarene films as sensitive coatings for QCMbased gas sensors. Sens. Actuators B Chem. 2005, 106, 177-181. [CrossRef]

175. D'Amico, A.; Pennazza, G.; Santonico, M.; Martinelli, E.; Roscioni, C.; Galluccio, G.; Paolesse, R.; Di Natale, C. An investigation on electronic nose diagnosis of lung cancer. Lung Cancer 2010, 68, 170-176. [CrossRef]

176. Santonico, M.; Pennazza, G.; Grasso, S.; D’Amico, A.; Bizzarri, M. Design and test of a Biosensor-based multisensorial system: A proof of concept study. Sensors 2013, 13, 16625-16640. [CrossRef]

177. Ippolito, S.J.; Ponzoni, A.; Kalantar-Zadeh, K.; Wlodarski, W.; Comini, E.; Faglia, G.; Sberveglieri, G. Layered WO $3 / \mathrm{ZnO} / 36^{\circ}$ $\mathrm{LiTaO}_{3} \mathrm{SAW}$ gas sensor sensitive towards ethanol vapour and humidity. Sens. Actuators B Chem. 2006, 117, 442-450. [CrossRef]

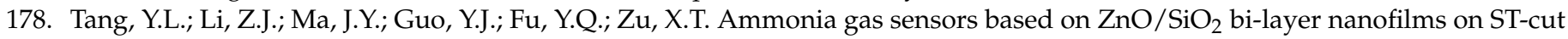
quartz surface acoustic wave devices. Sens. Actuators B Chem. 2014, 201, 114-121. [CrossRef] 
179. Tang, Y.; Ao, D.; Li, W.; Zu, X.; Li, S.; Fu, Y.Q. $\mathrm{NH}_{3}$ sensing property and mechanisms of quartz surface acoustic wave sensors deposited with $\mathrm{SiO}_{2}, \mathrm{TiO}_{2}$, and $\mathrm{SiO}_{2}-\mathrm{TiO}_{2}$ composite films. Sens. Actuators B Chem. 2018, 254, 1165-1173. [CrossRef]

180. Phillips, M.; Beatty, J.D.; Cataneo, R.N.; Huston, J.; Kaplan, P.D.; Lalisang, R.I.; Lambin, P.; Lobbes, M.B.I.; Mundada, M.; Pappas, N.; et al. Rapid point-of-care breath test for biomarkers of breast cancer and abnormal mammograms. PLoS ONE 2014, 9 , e90226. [CrossRef]

181. Phillips, M.; Basa-Dalay, V.; Blais, J.; Bothamley, G.; Chaturvedi, A.; Modi, K.D.; Pandya, M.; Natividad, M.P.R.; Patel, U.; Ramraje, N.N.; et al. Point-of-care breath test for biomarkers of active pulmonary tuberculosis. Tuberculosis 2012, 92, 314-320. [CrossRef] [PubMed]

182. Stahl, U.; Voigt, A.; Irschka, M.; Barié, N.; Richter, C.; Waldbaur, A.; Gruhl, F.J.; Rapp, B.E.; Rapp, M.; Länge, K. Long-term stability of polymer-coated surface transversewave sensors for the detection of organic solvent vapors. Sensors 2017, 17, 2529. [CrossRef] [PubMed]

183. Singh, P.; Yadava, R. Feature Extraction by Wavelet Decomposition of Surface. Def. Sci. J. 2010, 60, 377-386. [CrossRef]

184. Penza, M.; Antolini, F.; Vittori-Antisari, M. Carbon nanotubes-based surface acoustic waves oscillating sensor for vapour detection. Thin Solid Films 2005, 472, 246-252. [CrossRef]

185. Sayago, I.; Fernández, M.J.; Fontecha, J.L.; Horrillo, M.C.; Vera, C.; Obieta, I.; Bustero, I. New sensitive layers for surface acoustic wave gas sensors based on polymer and carbon nanotube composites. Sens. Actuators B Chem. 2012, 175, 67-72. [CrossRef]

186. David, M.; Arab, M.; Martino, C.; Delmas, L.; Guinneton, F.; Gavarri, J.R. Carbon nanotubes/ceria composite layers deposited on surface acoustic wave devices for gas detection at room temperature. Thin Solid Films 2012, 520, 4786-4791. [CrossRef]

187. Matatagui, D.; Kolokoltsev, O.; Saniger, J.M.; Gràcia, I.; Fernández, M.J.; Fontecha, J.L.; Horrillo, M.D.C. Acoustic sensors based on amino-functionalized nanoparticles to detect volatile organic solvents. Sensors 2017, 17, 2624. [CrossRef]

188. Yu, C.; Wu, Y.; Liu, X.; Fu, F.; Gong, Y.; Rao, Y.J.; Chen, Y. Miniature fiber-optic $\mathrm{NH}_{3}$ gas sensor based on Pt nanoparticleincorporated graphene oxide. Sens. Actuators B Chem. 2017, 244, 107-113. [CrossRef]

189. Kavinkumar, T.; Manivannan, S. Uniform decoration of silver nanoparticle on exfoliated graphene oxide sheets and its ammonia gas detection. Ceram. Int. 2016, 42, 1769-1776. [CrossRef]

190. Okuda, H.; Wang, T.; Lee, S.W. Selective Methanol Gas Detection Using a U-Bent Optical Fiber Modified with a Silica Nanoparticle Multilayer. Electron. Commun. Jpn. 2017, 100, 43-49. [CrossRef]

191. Manjula, M.; Karthikeyan, B.; Sastikumar, D. Sensing characteristics of nanocrystalline bismuth oxide clad-modified fiber optic gas sensor. Opt. Lasers Eng. 2017, 95, 78-82. [CrossRef]

192. Cha, J.H.; Kim, D.H.; Choi, S.J.; Koo, W.T.; Kim, I.D. Sub-Parts-per-Million Hydrogen Sulfide Colorimetric Sensor: Lead Acetate Anchored Nanofibers toward Halitosis Diagnosis. Anal. Chem. 2018, 90, 8769-8775. [CrossRef] [PubMed]

193. Zhong, X.; Li, D.; Du, W.; Yan, M.; Wang, Y.; Huo, D.; Hou, C. Rapid recognition of volatile organic compounds with colorimetric sensor arrays for lung cancer screening. Anal. Bioanal. Chem. 2018, 410, 3671-3681. [CrossRef] [PubMed]

194. Mazzone, P.J.; Hammel, J.; Dweik, R.; Na, J.; Czich, C.; Laskowski, D.; Mekhail, T. Diagnosis of lung cancer by the analysis of exhaled breath with a colorimetric sensor array. Thorax 2007, 62, 565-568. [CrossRef] [PubMed]

195. Cheng, C.S.; Chen, Y.Q.; Lu, C.J. Organic vapour sensing using localized surface plasmon resonance spectrum of metallic nanoparticles self assemble monolayer. Talanta 2007, 73, 358-365. [CrossRef]

196. Chen, B.; Liu, C.; Ge, L.; Hayashi, K. Localized surface plasmon resonance gas sensor of Au nano-islands coated with molecularly imprinted polymer: Influence of polymer thickness on sensitivity and selectivity. Sens. Actuators B Chem. 2016, 231, 787-792. [CrossRef]

197. Cui, J.; Hu, K.; Sun, J.J.; Qu, L.L.; Li, D.W. SERS nanoprobes for the monitoring of endogenous nitric oxide in living cells. Biosens. Bioelectron. 2016, 85, 324-330. [CrossRef]

198. Qiao, X.; Su, B.; Liu, C.; Song, Q.; Luo, D.; Mo, G.; Wang, T. Selective Surface Enhanced Raman Scattering for Quantitative Detection of Lung Cancer Biomarkers in Superparticle@MOF Structure. Adv. Mater. 2018, 30, 1702275. [CrossRef]

199. Zhang, Z.; Yu, W.; Wang, J.; Luo, D.; Qiao, X.; Qin, X.; Wang, T. Ultrasensitive Surface-Enhanced Raman Scattering Sensor of Gaseous Aldehydes as Biomarkers of Lung Cancer on Dendritic Ag Nanocrystals. Anal. Chem. 2017, 89, 1416-1420. [CrossRef] [PubMed]

200. Koureas, M.; Kirgou, P.; Amoutzias, G.; Hadjichristodoulou, C.; Gourgoulianis, K.; Tsakalof, A. Target analysis of volatile organic compounds in exhaled breath for lung cancer discrimination from other pulmonary diseases and healthy persons. Metabolites 2020, 10, 317. [CrossRef]

201. Rodríguez-Aguilar, M.; Díaz de León-Martínez, L.; Gorocica-Rosete, P.; Pérez-Padilla, R.; Domínguez-Reyes, C.A.; Tenorio-Torres, J.A.; Ornelas-Rebolledo, O.; Mehta, G.; Zamora-Mendoza, B.N.; Flores-Ramírez, R. Application of chemoresistive gas sensors and chemometric analysis to differentiate the fingerprints of global volatile organic compounds from diseases. Preliminary results of COPD, lung cancer and breast cancer. Clin. Chim. Acta 2021, 518, 83-92. [CrossRef] [PubMed]

202. van der Vliet, A.; Janssen-Heininger, Y.M.W.; Anathy, V. Oxidative stress in chronic lung disease: From mitochondrial dysfunction to dysregulated redox signaling. Mol. Aspects Med. 2018, 63, 59-69. [CrossRef] [PubMed]

203. Rodríguez-Aguilar, M.; Ramírez-García, S.; Ilizaliturri-Hernández, C.; Gómez-Gómez, A.; Van-Brussel, E.; Díaz-Barriga, F.; Medellín-Garibay, S.; Flores-Ramírez, R. Ultrafast gas chromatography coupled to electronic nose to identify volatile biomarkers in exhaled breath from chronic obstructive pulmonary disease patients: A pilot study. Biomed. Chromatogr. 2019, 33, e4684. [CrossRef] 
204. Chen, X.; Muhammad, K.G.; Madeeha, C.; Fu, W.; Xu, L.; Hu, Y.; Liu, J.; Ying, K.; Chen, L.; Yurievna, G.O. Calculated indices of volatile organic compounds (VOCs) in exhalation for lung cancer screening and early detection. Lung Cancer 2021, 154, 197-205. [CrossRef]

205. Schleich, F.N.; Zanella, D.; Stefanuto, P.; Bessonov, K.; Smolinska, A.; Dallinga, J.W.; Henket, M.; Paulus, V.; Guissard, F.; Graff, S.; et al. Exhaled Volatile Organic Compounds Are Able to Discriminate between Neutrophilic and Eosinophilic Asthma. Am. J. Respir. Crit. Care Med. 2019, 200, 444-453. [CrossRef]

206. Stefanuto, P.H.; Zanella, D.; Vercammen, J.; Henket, M.; Schleich, F.; Louis, R.; Focant, J.F. Multimodal combination of GC $\times$ GCHRTOFMS and SIFT-MS for asthma phenotyping using exhaled breath. Sci. Rep. 2020, 10, 16159. [CrossRef]

207. Moor, C.C.; Oppenheimer, J.C.; Nakshbandi, G.; Aerts, J.G.J.V.; Brinkman, P.; Maitland-Van Der Zee, A.H.; Wijsenbeek, M.S. Exhaled breath analysis by use of eNose technology: A novel diagnostic tool for interstitial lung disease. Eur. Respir. J. 2021, 57, 2002042. [CrossRef]

208. Buszewski, B.; Ligor, T.; Jezierski, T.; Wenda-Piesik, A.; Walczak, M.; Rudnicka, J. Identification of volatile lung cancer markers by gas chromatography-mass spectrometry: Comparison with discrimination by canines. Anal. Bioanal. Chem. 2012, 404, 141-146. [CrossRef]

209. Fuchs, P.; Loeseken, C.; Schubert, J.K.; Miekisch, W. Breath gas aldehydes as biomarkers of lung cancer. Int. J. Cancer 2010, 126, 2663-2670. [CrossRef] [PubMed]

210. Muñoz-Lucas, M.Á.; Jareño-Esteban, J.; Gutiérrez-Ortega, C.; López-Guijarro, P.; Collado-Yurrita, L.; Quintana-Díaz, M.; CallolSánchez, L. Influence of Chronic Obstructive Pulmonary Disease on Volatile Organic Compounds in Patients with Non-Small Cell Lung Cancer. Arch. Bronconeumol. 2020, 56, 801-805. [CrossRef]

211. Poli, D.; Carbognani, P.; Corradi, M.; Goldoni, M.; Acampa, O.; Balbi, B.; Bianchi, L.; Rusca, M.; Mutti, A. Exhaled volatile organic compounds in patients with non-small cell lung cancer: Cross sectional and nested short-term follow-up study. Respir. Res. 2005, 6, 71. [CrossRef] [PubMed]

212. Koureas, M.; Kalompatsios, D.; Amoutzias, G.D.; Hadjichristodoulou, C.; Gourgoulianis, K.; Tsakalof, A. Comparison of targeted and untargeted approaches in breath analysis for the discrimination of lung cancer from benign pulmonary diseases and healthy persons. Molecules 2021, 26, 2609. [CrossRef] [PubMed]

213. Zou, Y.; Zhang, X.; Chen, X.; Hu, Y.; Ying, K.; Wang, P. Optimization of volatile markers of lung cancer to exclude interferences of non-malignant disease. Cancer Biomarkers 2014, 14, 371-379. [CrossRef]

214. Fu, X.-A.; Li, M.; Knipp, R.J.; Nantz, M.H.; Bousamra, M. Noninvasive detection of lung cancer using exhaled breath. Cancer Med. 2014, 3, 174-181. [CrossRef]

215. Ii, M.B.; Schumer, E.; Li, M.; Knipp, R.J.; Nantz, M.H.; Van Berkel, V.; Fu, X. Quantitative analysis of exhaled carbonyl compounds distinguishes benign from malignant pulmonary disease. J. Thorac. Cardiovasc. Surg. 2014, 148, 1074-1081. [CrossRef]

216. Li, M.; Yang, D.; Brock, G.; Knipp, R.J.; Bousamra, M.; Nantz, M.H.; Fu, X.A. Breath carbonyl compounds as biomarkers of lung cancer. Lung Cancer 2015, 90, 92-97. [CrossRef]

217. Ibrahim, B.; Basanta, M.; Cadden, P.; Singh, D.; Douce, D.; Woodcock, A.; Fowler, S.J. Non-invasive phenotyping using exhaled volatile organic compounds in asthma. Thorax 2011, 66, 804-809. [CrossRef]

218. Basanta, M.; Ibrahim, B.; Dockry, R.; Douce, D.; Morris, M.; Singh, D.; Woodcock, A.; Fowler, S.J. Exhaled volatile organic compounds for phenotyping chronic obstructive pulmonary disease: A cross-sectional study. Respir. Res. 2012, 13, 72. [CrossRef]

219. Van Vliet, D.; Smolinska, A.; Jöbsis, Q.; Rosias, P.P.R.; Muris, J.W.M.; Dallinga, J.W.; Van Schooten, F.J.; Dompeling, E. Association between exhaled inflammatory markers and asthma control in children. J. Breath Res. 2016, 10, 016014. [CrossRef] [PubMed]

220. Van Vliet, D.; Smolinska, A.; Jöbsis, Q.; Rosias, P.; Muris, J.; Dallinga, J.; Dompeling, E.; Van Schooten, F.J. Can exhaled volatile organic compounds predict asthma exacerbations in children? J. Breath Res. 2017, 11, 016016. [CrossRef] [PubMed]

221. Robroeks, C.M.; Van Berkel, J.J.; Jöbsis, Q.; Van Schooten, F.J.; Dallinga, J.W.; Wouters, E.F.; Dompeling, E. Exhaled volatile organic compounds predict exacerbations of childhood asthma in a 1-year prospective study. Eur. Respir. J. 2013, 42, 98-106. [CrossRef] [PubMed]

222. Pizzini, A.; Filipiak, W.; Wille, J.; Ager, C.; Wiesenhofer, H.; Kubinec, R.; Blaško, J.; Tschurtschenthaler, C.; Mayhew, C.A.; Weiss, G.; et al. Analysis of volatile organic compounds in the breath of patients with stable or acute exacerbation of chronic obstructive pulmonary disease. J. Breath Res. 2018, 12, 036002. [CrossRef] [PubMed]

223. Dragonieri, S.; Annema, J.T.; Schot, R.; Van Der Schee, M.P.C.; Spanevello, A.; Carratú, P.; Resta, O.; Rabe, K.F.; Sterk, P.J. An electronic nose in the discrimination of patients with non-small cell lung cancer and COPD. Lung Cancer 2009, 64, 166-170. [CrossRef]

224. Tirzite, M.; Bukovskis, M.; Strazda, G.; Jurka, N.; Taivans, I. Detection of lung cancer in exhaled breath with an electronic nose using support vector machine analysis. J. Breath Res. 2017, 11, 036009. [CrossRef] [PubMed]

225. Tirzite, M.; Bukovskis, M.; Strazda, G.; Jurka, N.; Taivans, I. Detection of lung cancer with electronic nose and logistic regression analysis. J. Breath Res. 2019, 13, 016006. [CrossRef]

226. Binson, V.A.; Subramoniam, M.; Sunny, Y.; Mathew, L. Prediction of Pulmonary Diseases with Electronic Nose Using SVM and XGBoost. IEEE Sens. J. 2021, 21, 20886-20895. [CrossRef]

227. Chen, K.; Liu, L.; Nie, B.; Lu, B.; Fu, L.; He, Z.; Li, W.; Pi, X.; Liu, H. Recognizing lung cancer and stages using a self-developed electronic nose system. Comput. Biol. Med. 2021, 131, 104294. [CrossRef] 
228. Shlomi, D.; Abud, M.; Liran, O.; Bar, J.; Gai-Mor, N.; Ilouze, M.; Onn, A.; Ben-Nun, A.; Haick, H.; Peled, N. Detection of Lung Cancer and EGFR Mutation by Electronic Nose System. J. Thorac. Oncol. 2017, 12, 1544-1551. [CrossRef] [PubMed]

229. Fens, N.; Zwinderman, A.H.; Van Der Schee, M.P.; De Nijs, S.B.; Dijkers, E.; Roldaan, A.C.; Cheung, D.; Bel, E.H.; Sterk, P.J Exhaled Breath Profiling Enables Discrimination of Chronic Obstructive Pulmonary Disease and Asthma. Am. J. Respir. Crit. Care Med. 2009, 180, 1076-1082. [CrossRef] [PubMed]

230. Fens, N.; Roldaan, A.C.; van der Schee, M.P.; Boksem, R.J.; Zwinderman, A.H.; Bel, E.H.; Sterk, P.J. External validation of exhaled breath profiling using an electronic nose in the discrimination of asthma with fixed airways obstruction and chronic obstructive pulmonary disease. Clin. Exp. Allergy 2011, 41, 1371-1378. [CrossRef]

231. Dragonieri, S.; Scioscia, G.; Quaranta, V.N.; Carratu, P.; Venuti, M.P.; Falcone, M.; Carpagnano, G.E.; Foschino Barbaro, M.P.; Resta, O.; Lacedonia, D. Exhaled volatile organic compounds analysis by e-nose can detect idiopathic pulmonary fibrosis. J. Breath Res. 2020, 14, 047101. [CrossRef]

232. Fielding, D.; Hartel, G.; Pass, D.; Davis, M.; Brown, M.; Dent, A.; Agnew, J.; Dickie, G.; Ware, R.S.; Hodge, R. Volatile organic compound breath testing detects in-situ squamous cell carcinoma of bronchial and laryngeal regions and shows distinct profiles of each tumour. J. Breath Res. 2020, 14, 046013. [CrossRef]

233. Bach, J.P.; Gold, M.; Mengel, D.; Hattesohl, A.; Lubbe, D.; Schmid, S.; Tackenberg, B.; Rieke, J.; Maddula, S.; Baumbach, J.I.; et al. Measuring compounds in exhaled air to detect Alzheimer's disease and Parkinson's disease. PLoS ONE 2015, 10, e0132227. [CrossRef] [PubMed]

234. Krauss, E.; Haberer, J.; Maurer, O.; Barreto, G.; Drakopanagiotakis, F.; Degen, M.; Seeger, W.; Guenther, A. Exploring the ability of electronic nose technology to recognize interstitial lung diseases (ILD) by non-invasive breath screening of exhaled volatile compounds (VOC): A pilot study from the european ipf registry (eurIPFreg) and biobank. J. Clin. Med. 2019, 8, 1698. [CrossRef]

235. Bannier, M.A.G.E.; Van De Kant, K.D.G.; Jöbsis, Q.; Dompeling, E. Feasibility and diagnostic accuracy of an electronic nose in children with asthma and cystic fibrosis. J. Breath Res. 2019, 13, 36009. [CrossRef]

236. van Hooren, M.R.A.; Leunis, N.; Brandsma, D.S.; Dingemans, A.M.C.; Kremer, B.; Kross, K.W. Differentiating head and neck carcinoma from lung carcinoma with an electronic nose: A proof of concept study. Eur. Arch. Oto-Rhino-Laryngol. 2016, 273, 3897-3903. [CrossRef]

237. van de Goor, R.M.G.E.; Leunis, N.; van Hooren, M.R.A.; Francisca, E.; Masclee, A.; Kremer, B.; Kross, K.W. Feasibility of electronic nose technology for discriminating between head and neck, bladder, and colon carcinomas. Eur. Arch. Oto-Rhino-Laryngol. 2017, 274, 1053-1060. [CrossRef]

238. Barash, O.; Zhang, W.; Halpern, J.M.; Hua, Q.L.; Pan, Y.Y.; Kayal, H.; Khoury, K.; Liu, H.; Davies, M.P.A.; Haick, H. Differentiation between genetic mutations of breast cancer by breath volatolomics. Oncotarget 2015, 6, 44864-44876. [CrossRef]

239. Amal, H.; Leja, M.; Funka, K.; Skapars, R.; Sivins, A.; Ancans, G.; Liepniece-karele, I.; Kikuste, I.; Lasina, I.; Haick, H. Detection of precancerous gastric lesions and gastric cancer through exhaled breath. Gut 2016, 65, 400-407. [CrossRef] [PubMed]

240. Xu, Z.; Broza, Y.Y.; Ionsecu, R.; Tisch, U.; Ding, L.; Liu, H.; Song, Q.; Pan, Y.; Xiong, F.; Gu, K. A nanomaterial-based breath test for distinguishing gastric cancer from benign gastric conditions. Br. J. Cancer 2013, 108, 941-950. [CrossRef] [PubMed]

241. Daniel, D.; Thangavel, K. Breathomics for gastric cancer classification using back-propagation neural network. J. Med. Signals Sens. 2016, 6, 172-182. [CrossRef] [PubMed]

242. Raspagliesi, F.; Bogani, G.; Benedetti, S.; Grassi, S.; Ferla, S.; Buratti, S. Detection of ovarian cancer through exhaled breath by electronic nose: A prospective study. Cancers 2020, 12, 2408. [CrossRef] [PubMed]

243. Lau, H.C.; Yu, J.B.; Lee, H.W.; Huh, J.S.; Lim, J.O. Investigation of exhaled breath samples from patients with Alzheimer's disease using gas chromatography-mass spectrometry and an exhaled breath sensor system. Sensors 2017, 17, 1783. [CrossRef] [PubMed]

244. Pennazza, G.; Santonico, M.; Vernile, C.; Antonelli Incalzi, R.; De Vincentis, A.; Picardi, A. Breathprinting of liver diseases. In Proceedings of the 2015 18th AISEM Annual Conference, Trento, Italy, 3-5 February 2015; pp. 1-3. [CrossRef]

245. Saidi, T.; Zaim, O.; Moufid, M.; El Bari, N.; Ionescu, R.; Bouchikhi, B. Exhaled breath analysis using electronic nose and gas chromatography-mass spectrometry for non-invasive diagnosis of chronic kidney disease, diabetes mellitus and healthy subjects. Sens. Actuators B Chem. 2018, 257, 178-188. [CrossRef]

246. Saidi, T.; Moufid, M.; de Jesus Beleño-Saenz, K.; Welearegay, T.G.; El Bari, N.; Lisset Jaimes-Mogollon, A.; Ionescu, R.; Bourkadi, J.E.; Benamor, J.; El Ftouh, M.; et al. Non-invasive prediction of lung cancer histological types through exhaled breath analysis by UV-irradiated electronic nose and GC/QTOF/MS. Sens. Actuators B Chem. 2020, 311, 127932. [CrossRef]

247. Plaza, V.; Crespo, A.; Giner, J.; Merino, J.L.; Ramos-Barbón, D.; Mateus, E.F.; Torrego, A.; Cosio, B.G.; Agustí, A.; Sibila, O. Inflammatory asthma phenotype discrimination using an electronic nose breath analyzer. J. Investig. Allergol. Clin. Immunol. 2015, 25, 431-437.

248. Farraia, M.; Rufo, J.C.; Paciência, I.; Castro, F.; Ana, M.; Rama, T.; Rocha, S.M.; Delgado, L.; Brinkman, P.; Moreira, A. Human volatilome analysis using eNose to assess uncontrolled asthma in a clinical setting. Allergy 2020, 75, 1630-1639. [CrossRef]

249. Phillips, M.; Cataneo, R.N.; Ditkoff, B.A.; Fisher, P.; Greenberg, J.; Gunawardena, R.; Kwon, C.S.; Tietje, O.; Wong, C. Prediction of breast cancer using volatile biomarkers in the breath. Breast Cancer Res. Treat. 2006, 99, 19-21. [CrossRef] [PubMed]

250. Tiele, A.; Daulton, E.; Ifeachor, E.; Eyre, V.; Clarke, S. Breath-based non-invasive diagnosis of Alzheimer' s disease : A pilot study Breath-based non-invasive diagnosis of Alzheimer's disease: A pilot study. J. Breath Res. 2020, 14, 026003. [CrossRef]

251. Das, S.; Pal, M. Review-Non-Invasive Monitoring of Human Health by Exhaled Breath Analysis: A Comprehensive Review. J. Electrochem. Soc. 2020, 167, 037562. [CrossRef] 
252. Sakumura, Y.; Koyama, Y.; Tokutake, H.; Hida, T.; Sato, K.; Itoh, T.; Akamatsu, T.; Shin, W. Diagnosis by volatile organic compounds in exhaled breath from lung cancer patients using support vector machine algorithm. Sensors 2017, 17, 287. [CrossRef] [PubMed] 